\title{
A direct elimination algorithm for quasi-static and dynamic contact problems
}

\author{
D. Di Capua ${ }^{1,3}$, C. Agelet de Saracibar ${ }^{2,3}$ \\ ${ }^{I}$ Escola Universitària d'Enginyeria Tècnica Industrial (EUETIB), Universidad Politécnica de Cataluña (UPC), \\ Comte d'Urgell 187, 08036 Barcelona, Spain \\ ${ }^{2}$ ETS Ingenieros de Caminos, Canales y Puertos, Universidad Politécnica de Cataluña (UPC), \\ Edificio C1, Campus Norte, UPC, Jordi Girona 1-3, 08034 Barcelona, Spain \\ ${ }^{3}$ International Center for Numerical Methods in Engineering (CIMNE), Gran Capitán s/n, 08034 Barcelona, Spain \\ dicapua@cimne.upc.edu, agelet@cimne.upc.edu
}

22 June 2014

Submitted to

Finite Elements in Analysis and Design 


\title{
A direct elimination algorithm for quasi-static and dynamic contact problems
}

\author{
D. Di Capua ${ }^{1,3}$, C. Agelet de Saracibar ${ }^{2,3}$ \\ ${ }^{I}$ Escola Universitària d'Enginyeria Tècnica Industrial (EUETIB), Universidad Politécnica de Cataluña, UPC \\ BarcelonaTech, Comte d'Urgell 187, 08036 Barcelona, Spain \\ ${ }^{2}$ ETS Ingenieros de Caminos, Canales y Puertos, Universidad Politécnica de Cataluña, UPC BarcelonaTech \\ Edificio C1, Campus Norte, UPC, Jordi Girona 1-3, 08034 Barcelona, Spain \\ ${ }^{3}$ International Center for Numerical Methods in Engineering (CIMNE), Gran Capitán s/n, 08034 Barcelona, Spain
}

dicanua@cimne.unc.edu,agelet@cimne.unc.edu

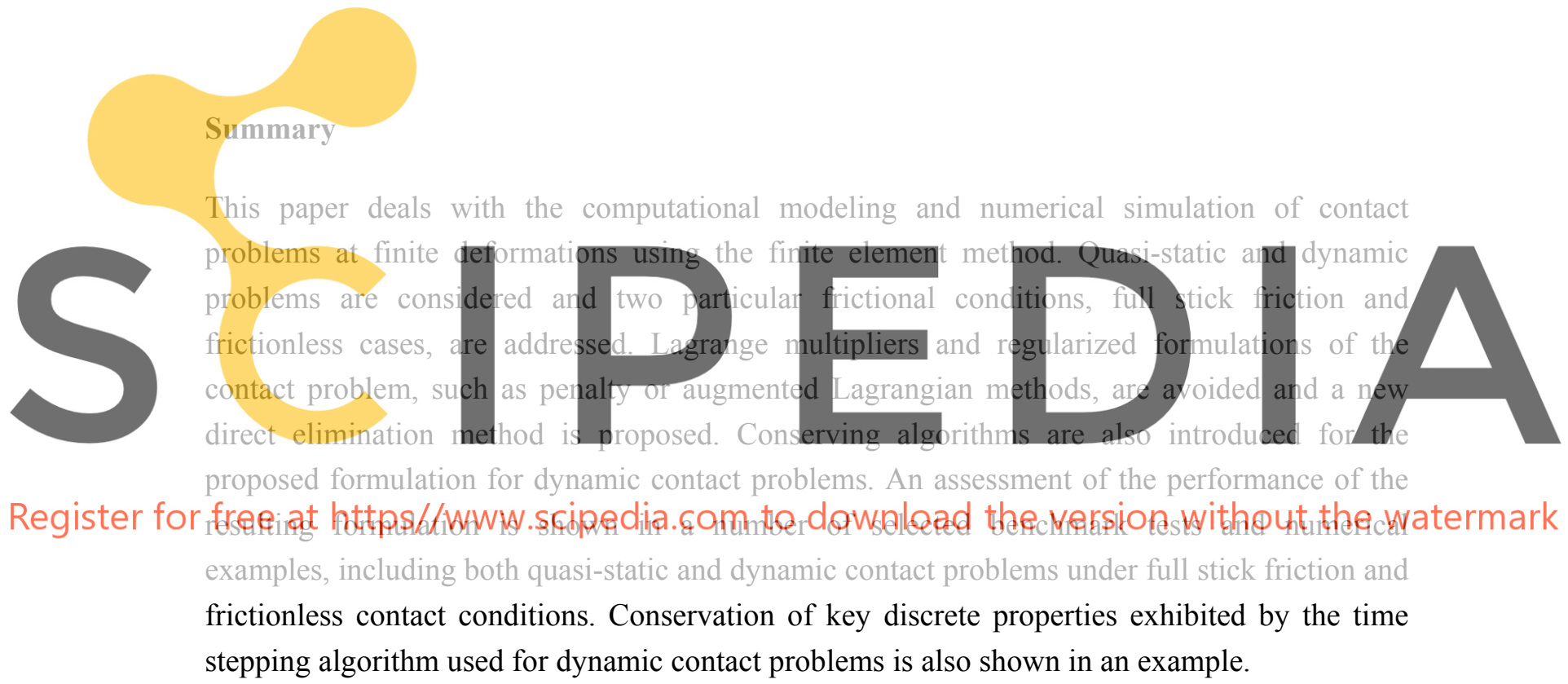

Version 6.0: 22 June 2014

Keywords: contact mechanics, full stick friction, frictionless, finite elements, dynamics, conserving algorithms

\section{Introduction, motivation and goals}

Numerical analysis of contact problems has been one of the hot research topics of interest over the last decades. Contact problems arise in many applications, such as in crashworthiness, projectile impact, and material forming processes, i.e. sheet metal forming, bulk forming, casting, friction stir welding, cutting, and powder compaction. Despite the important progresses achieved in computational contact mechanics, the numerical simulation of contact problems is still nowadays a complex task, mainly due to the highly nonlinear nature of the problem, potentially involving fully nonlinear kinematics, finite strains, large slips, nonlinear boundary conditions, complex frictional behavior phenomena, thermomechanical contact, etc. 
Mathematically, the numerical analysis of a frictional contact problem amounts to finding the solution of an Initial Boundary Value Problem (IBVP) within a constrained solution space. The variational formulation of a frictional contact problem includes restrictions on the admissible variations in the tangent solution space induced by the contact constraints, yielding to Variational Inequalities (VI). See, for instance, Kikuchi \& Oden (1988) [27] and Duvaut \& Lions (1972) [19].

A regularization of the frictional contact constraints, using penalty or augmented Lagrangian methods, allows us to bypass the need to find a solution within a constrained solution space and provides a very convenient displacement driven frictional contact formulation.

The penalty method can be considered as the standard regularization procedure for computational modeling of frictional contact problems and it has been widely used, for instance, by Oden \& Pires (1984) [41], Oden \& Martins (1985) [42], Hallquist, Goudreau \& Benson (1985) [22], Curnier \& Alart (1988) [18], Benson \& Hallquist (1990) [11], Wriggers, Vu Van \& Stein (1990) [62], Belytschko \& Neal (1991) [10], Laursen (1992, 1999, 2002) [30,35,37], Laursen \& Simo (1991, 1992, 1993) [29,31,32], Agelet de Saracibar $(1997,1998)$ [1,2], Petocz (1998) [44], Armero \& Petocz (1998, 1999) [6,7], Agelet de Saracibar \& Chiumenti (1999) [4], Agelet de Saracibar, Cervera \& Chiumenti $(1999,2001)$ [3,5], and Chiumenti, Agelet de Saracibar \& Cervera (2008) [17].
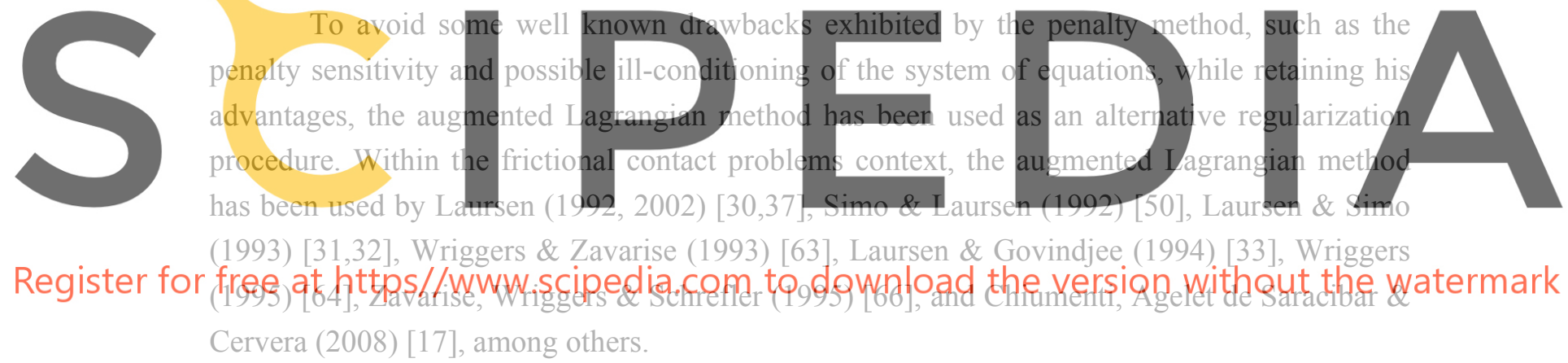

A perturbed Lagrangian method has been used, for instance, by Simo, Wriggers \& Taylor (1985) [56] and Ju \& Taylor (1988) [26].

A displacement driven formulation of the frictional contact problem allows to widely exploit the features of the framework developed for computational plasticity. See, for instance, Simo \& Hughes (1998) [57] and Simo (1994) [53] for an excellent account of computational plasticity. In particular, return mapping algorithms developed for plasticity can be applied to integrate the frictional contact traction. The lowest order member of the family of backwarddifference (BD) methods, the backward-Euler (BE) time integration algorithm, has become the standard frictional return mapping algorithm for the regularized frictional contact constrained evolution problem. Frictional return mapping algorithms using the BE method have been used by Giannakopoulos (1989) [20], Wriggers, Vu Van \& Stein (1990) [62], Laursen \& Simo (1993) [31,32], Agelet de Saracibar (1997, 1998) [1,2], Agelet de Saracibar \& Chiumenti (1999) [4], and Agelet de Saracibar, Cervera \& Chiumenti $(1999,2001)$ [3,5], among others. Within the family of Implicit Runge-Kutta (IRK) methods, a generalized Projected Mid-Point (PMP) algorithm, initially proposed by Simo (1994) [53] for computational plasticity, has been 
proposed by Agelet de Saracibar (1998) [2] as frictional return mapping algorithm for the time integration of the frictional traction problem.

Typically, within the framework of the Finite Element (FE) method, most of the discrete frictional contact problems are formulated using the local parametrization induced by the $\mathrm{FE}$ triangularization of the contact surfaces. Due to the local character of the parametrization, the frictional time integration algorithm may turn out to be useless if large slips are involved. A new frictional time integration algorithm, designed to avoid the drawbacks arising from a local parametrization, being suitable for large slip multi-body frictional contact problems in 2D and 3D, has been developed by Agelet de Saracibar (1997) [1]. Time integration of the frictional traction is performed using Hermite interpolation functions and introducing a new slip path parametrization, which, remarkably, is defined on the sole basis of the outward unit normal to the master surface, being independently of the local surface finite element parametrization used in the spatial triangularization.

A pinball algorithm for contact-impact problems, using penalty and Lagrangian methods, has been presented by Belytschko \& Neal (1991) [10].

It is well known that node-to-segment (NTS) contact formulations do not pass the contact patch test [47]. An alternative to the node-segment contact formulation is the mortar segment-to-segment contact formulation. A mortar segment-to segment contact formulation for large deformation solid mechanics has been presented by Puso \& Laursen $(2004)[45,46]$ and

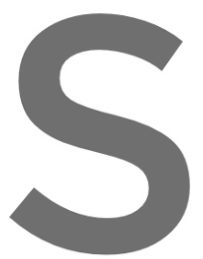
Yang (2006) [65]. Dual active set strategies bas
Wohlmuth (2000) [59], Hüeber \& Wohlmuth (2
Isogeometric A nalysis (IGA) was recent
(2005) [25]. Within the IGA framework, the sa
NURBS, are used fo both the CAD geometry at
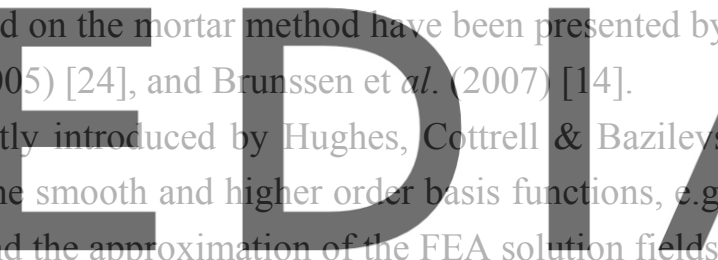

leading to evident potential advantages in the description of interacting surfaces undergoing Reg ister for free at https/fwWW scipedia.com to download the version without the watermark

detailed and up-to-date review of isogeometric contact formulations can be found in Lorenzis, Wriggers \& Hughes (2014) [38].

A computational model for frictionless contact problems using the null-space method and introducing a smoothing technique of the master surface using cubic B-spline interpolation has been presented by Muñoz (2008) [40].

Time discrete conserving algorithms for nonlinear dynamics have been proposed by Simo \& Wong (1991) [49], Simo \& Tarnow (1992) [51], Simo, Tarnow \& Wong (1992) [52], Simo \&Tarnow (1994) [54], Simo, Tarnow \& Doblaré (1995) [55], Gonzalez (2000) [21], Laursen \& Meng (2001) [36], Armero \& Romero (2001) [8], Meng \& Laursen (2002) [39], and Armero (2008) [9]. The extension of time discrete conserving algorithms for frictionless and frictional contact problems has been done by Chawla (1997) [15], Laursen \& Chawla (1997) [34], Chawla \& Laursen (1998) [16], Petocz (1998) [44], and Armero \& Petocz (1998, 1999) [6,7], among others. Bravo, Pérez Aparicio \& Laursen (2011) [13] have proposed an Enhanced Energy Conserving Algorithm (EECA) formulation for time integration of frictionless contactimpact problems using an enhanced penalty method, featuring energy, linear and angular momentum conservation. Energy consistent time stepping schemes for finite-dimensional mechanical systems with holonomic constraints have been presented by Betsch (2005) [12]. 
A fully nonlinear kinematics formulation of frictionless contact problems, including the derivation of the algorithmic contact operators, was presented by Wriggers \& Simo (1985) [59] for 2D problems using linear surface elements, and by Parisch (1989) [43] for 3D problems using linear surface elements. An extension of the formulation to frictional contact problems was provided by Wriggers (1987) [61]. A general fully nonlinear kinematics formulation for multi-body frictional contact problems at finite strains in 2D and 3D, was first developed on a continuum setting by Laursen \& Simo (1993) [31]. The fully nonlinear kinematics formulation of frictional contact problems developed by Laursen \& Simo (1993) [31] was extended later on by Agelet de Saracibar (1998) [2] for coupled thermomechanical problems, Agelet de Saracibar \& Chiumenti (1999) [4] to account for wear phenomena, and Agelet de Saracibar, Cervera \& Chiumenti $(1999,2001)[3,5]$ to account for coupled thermoplastic problems including phasechange.

This paper deals with the computational modeling and numerical simulation of contact problems at finite deformations using the finite element method. Quasi-static and dynamic problems are considered and two particular frictional conditions, full stick and frictionless cases, are addressed. Lagrange multipliers and regularized formulations of the contact problem, such as penalty or augmented Lagrangian methods, are avoided and a new direct elimination method is proposed. A conserving algorithm is used for dynamic contact poblems.

The outline of the paper is as follows. In Section 2 we present the continuum

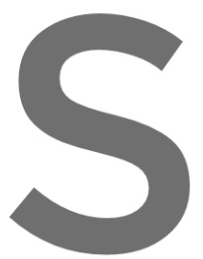
formulation of the contact problem. Section 3 deals with the finite
contactless problem. Section 4 deals with the finite element form
of the full stick and frictionless contact cases, using the propose
Finally, Section 5 deals with an assessment of the contact forn
number of representative quasi static and dynamic numerical ex

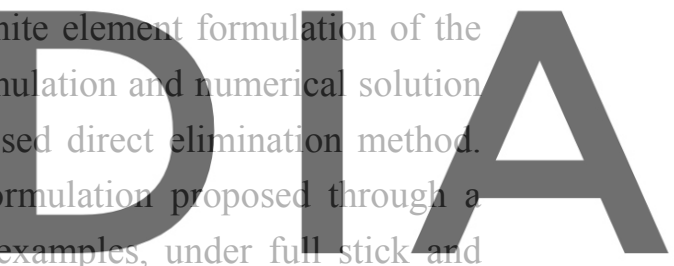

frictionless contact conditions. The paper concludes with some final remarks. An Appendix, Register for free at httos/LWWW:scipedia. com to d download the version without the watermark point-projection on the master surface, has been also included.

\section{Continuum formulation of the contact mechanics problem}

\subsection{Local formulation}

Let $2 \leq n_{\text {dim }} \leq 3$ be the space dimension and $\mathbb{I}:=[0, T] \subset \mathbb{R}_{+}$the time interval of interest. Let the open sets $\Omega^{(1)} \subset \mathbb{R}^{n_{\text {dim }}}$ and $\Omega^{(2)} \subset \mathbb{R}^{n_{\text {dim }}}$, with smooth boundaries $\partial \Omega^{(1)}$ and $\partial \Omega^{(2)}$ and closures $\bar{\Omega}^{(1)}=\Omega^{(1)} \cup \partial \Omega^{(1)}$ and $\bar{\Omega}^{(2)}=\Omega^{(2)} \cup \partial \Omega^{(2)}$, be the reference placement of two continuum bodies $\mathcal{B}^{(1)}$ and $\mathcal{B}^{(2)}$.

For each body $\mathcal{B}^{(i)}$ we denote by $\mathbf{X}^{(i)} \in \bar{\Omega}^{(i)}$ the vector position of the material particles at the reference configuration, $\varphi^{(i)}: \bar{\Omega}^{(i)} \times \mathbb{I} \rightarrow \mathbb{R}^{n_{\text {dim }}}$ the orientation preserving deformation maps, $\mathbf{V}^{(i)}:=\partial_{t} \boldsymbol{\varphi}^{(i)}$ the material velocities, $\mathbf{u}^{(i)}:=\boldsymbol{\varphi}^{(i)}-\mathbf{X}^{(i)}$ the material displacements, $\rho_{0}^{(i)}$ the reference mass densities, and $\mathbf{F}^{(i)}:=\operatorname{GRAD} \varphi^{(i)}$ the deformation gradients, where GRAD denotes the material gradient operator. For each time $t \in \mathbb{I}$, the mapping $t \in \mathbb{I} \mapsto \varphi_{t}^{(i)}:=\varphi^{(i)}(\cdot, t)$ represents a one-parameter family of configurations indexed by time $t$, which maps the reference placement of body $\mathcal{B}^{(i)}$ onto its current placement 
$\mathcal{S}_{t}^{(i)}: \varphi_{t}^{(i)}\left(\mathcal{B}^{(i)}\right) \subset \mathbb{R}^{n_{\mathrm{dim}}}$. The current placement of particles $\mathbf{X}^{(i)} \in \bar{\Omega}^{(i)}$ at time $t \in \mathbb{I}$ is denoted as $\mathbf{x}^{(i)}:=\varphi^{(i)}\left(\mathbf{X}^{(i)}, t\right)$.

We will asume that no contact forces are present between the two bodies at the reference configuration. Subsequent configurations cause the two bodies to physically come into contact and produce contact interactive forces during some portion of the time interval of interest $\mathbb{I}:=[0, T] \subset \mathbb{R}_{+}$.

For each body $\mathcal{B}^{(i)}$ we will consider the following partitions of the boundary $\partial \Omega^{(i)}=\partial_{u} \Omega^{(i)} \cup \partial_{\sigma} \Omega^{(i)} \cup \partial_{c} \Omega^{(i)}$, where $\partial_{u} \Omega^{(i)}, \partial_{\sigma} \Omega^{(i)}$ and $\Gamma_{c}^{(i)}:=\partial_{c} \Omega^{(i)}$ represent the prescribed displacements, prescribed nominal tractions and contact boundaries, respectively, such that the conditions $\varnothing=\partial_{u} \Omega^{(i)} \cap \partial_{\sigma} \Omega^{(i)}, \varnothing=\partial_{u} \Omega^{(i)} \cap \partial_{c} \Omega^{(i)}$ and $\varnothing=\partial_{\sigma} \Omega^{(i)} \cap \partial_{c} \Omega^{(i)}$, hold.

The local material form of the momentum balance equation, prescribed traction and prescribed displacement boundary conditions, and initial conditions for body $\mathcal{B}^{(i)}$ take the form,

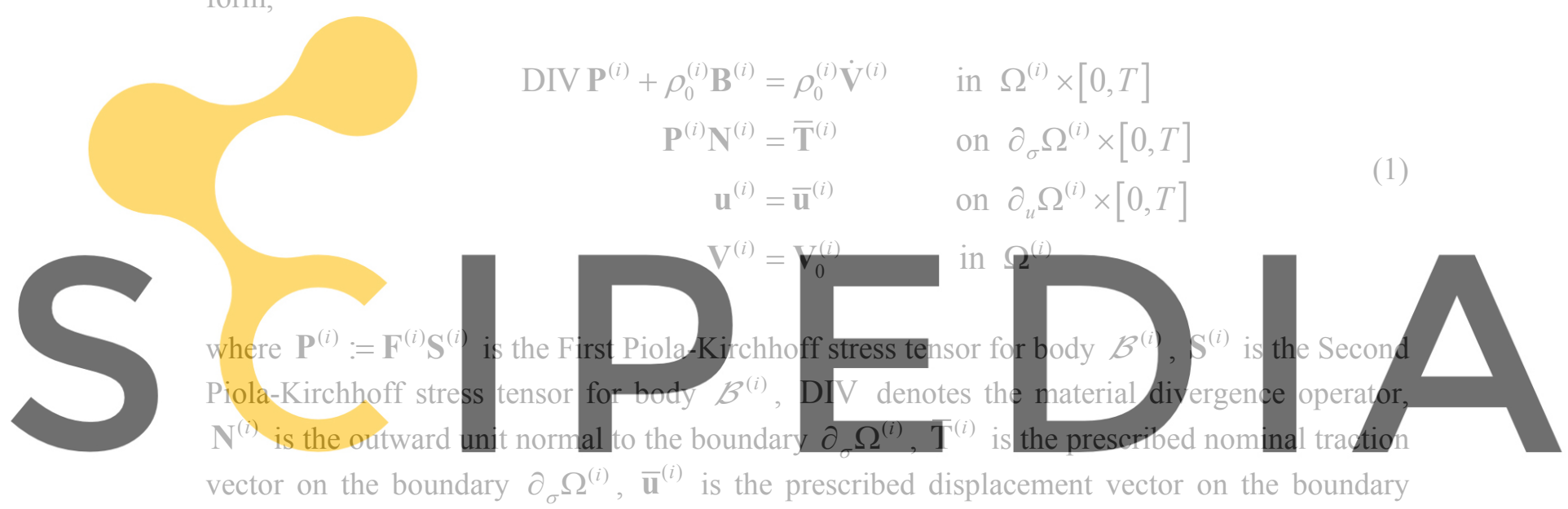
derivative.

Assuming a linear Saint-Venant Kirchhoff elastic constitutive model for the body $\mathcal{B}^{(i)}$, the free energy per unit of mass can be expressed as,

$$
\Psi^{(i)}\left(\mathbf{E}^{(i)}\right)=\frac{1}{2 \rho_{0}} \mathbf{E}^{(i)}: \mathbb{C}^{(i)}: \mathbf{E}^{(i)}
$$

where $\mathbb{C}^{(i)}$ is the constant fourth order elastic constitutive tensor and $\mathbf{E}^{(i)}$ is the GreenLagrange strain tensor.

\subsection{Local formulation of the contact problem}

Using the classical slave-master formulation of contact mechanics [1,2,29-33,37], let us denote the contact surfaces $\Gamma_{c}^{(1)}$ and $\Gamma_{c}^{(2)}$ as slave and master contact surfaces, respectively. Particles of the slave and master contact surfaces will be denoted as slave particles and master particles, respectively.

Let us consider a slave particle $\mathbf{X}^{(1)} \in \Gamma_{c}^{(1)}$, being $\mathbf{x}^{(1)}=\boldsymbol{\varphi}^{(1)}\left(\mathbf{X}^{(1)}, t\right) \in \gamma_{c}^{(1)}$ its current spatial vector position of the slave particle at time $t \in \mathbb{I}$, and $\mathbf{y}\left(\mathbf{X}^{(1)}, t\right) \in \gamma_{c}^{(2)}$ its closest-point- 
projection onto the spatial configuration $\gamma_{c}^{(2)}$ of the master surface $\Gamma_{c}^{(2)}$ at time $t \in \mathbb{I}$, defined as,

$$
\begin{aligned}
& \overline{\mathbf{y}}\left(\mathbf{X}^{(1)}, t\right):=\boldsymbol{\varphi}^{(2)}\left(\overline{\mathbf{Y}}\left(\mathbf{X}^{(1)}, t\right), t\right) \\
& \overline{\mathbf{Y}}\left(\mathbf{X}^{(1)}, t\right):=\arg \min _{\mathbf{X}^{(2)} \in \Gamma_{c}^{(2)}}\left\|\boldsymbol{\varphi}^{(1)}\left(\mathbf{X}^{(1)}, t\right)-\boldsymbol{\varphi}^{(2)}\left(\mathbf{X}^{(2)}, t\right)\right\|
\end{aligned}
$$

The contact normal gap function $g_{N}\left(\mathbf{X}^{(1)}, t\right)$ for a slave particle $\mathbf{X}^{(1)} \in \Gamma_{c}^{(1)}$ at time $t \in \mathbb{I}$ is defined as,

$$
g_{N}\left(\mathbf{X}^{(1)}, t\right):=\left(\varphi^{(1)}\left(\mathbf{X}^{(1)}, t\right)-\varphi^{(2)}\left(\overline{\mathbf{Y}}\left(\mathbf{X}^{(1)}, t\right), t\right)\right) \cdot \mathbf{n}
$$

where $\mathbf{n}$ is the outward unit normal to the spatial configuration of the master surface at the closest-point-projection $\overline{\mathbf{y}}\left(\mathbf{X}^{(1)}, t\right) \in \gamma_{c}^{(2)}$. Assuming enough smooth contact surfaces, it is assumed that the following condition holds,

$$
\mathbf{n}=\mathbf{n}^{(2)}\left(\overline{\mathbf{Y}}\left(\mathbf{X}^{(1)}, t\right), t\right)=-\mathbf{n}^{(1)}\left(\mathbf{X}^{(1)}, t\right)
$$

The nominal frictional contact vector $\mathbb{T}^{(1)}\left(\mathbf{X}^{(1)}, t\right)$ at a slave particle $\mathbf{X}^{(1)} \in \Gamma_{c}^{(1)}$ at time
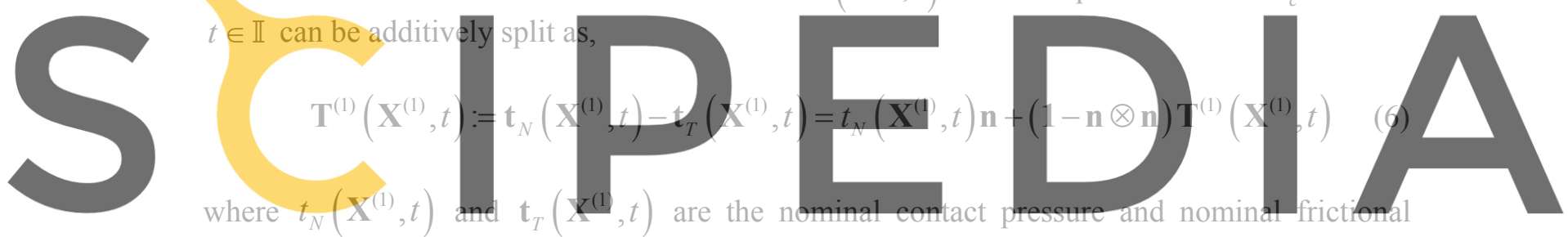

tangent traction vector, respectively

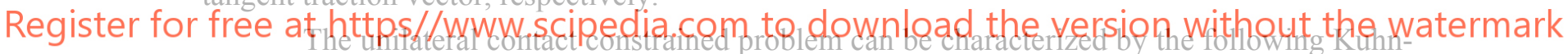

Tucker and contact persistency conditions [1,2,29-33,37]:

$$
\begin{gathered}
g_{N}\left(\mathbf{X}^{(1)}, t\right) \geq 0, \quad t_{N}\left(\mathbf{X}^{(1)}, t\right) \geq 0, \quad t_{N}\left(\mathbf{X}^{(1)}, t\right) g_{N}\left(\mathbf{X}^{(1)}, t\right)=0 \\
\text { if } \quad g_{N}\left(\mathbf{X}^{(1)}, t\right)=0 \quad \text { then } \quad t_{N}\left(\mathbf{X}^{(1)}, t\right) \dot{g}_{N}\left(\mathbf{X}^{(1)}, t\right)=0
\end{gathered}
$$

where

$$
\dot{g}_{N}\left(\mathbf{X}^{(1)}, t\right):=\left(\mathbf{V}^{(1)}\left(\mathbf{X}^{(1)}, t\right)-\mathbf{V}^{(2)}\left(\overline{\mathbf{Y}}\left(\mathbf{X}^{(1)}, t\right), t\right)\right) \cdot \mathbf{n}
$$

\subsection{Variational formulation}

The variational form of the momentum balance equation for a problem involving contact between two bodies $\mathcal{B}^{(1)}$ and $\mathcal{B}^{(2)}$ can be written as [1,2,29-33,37],

$$
\begin{aligned}
& \sum_{i=1}^{2}\left(\rho_{0}^{(i)} \dot{\mathbf{V}}^{(i)}, \boldsymbol{\eta}^{(i)}\right)+\sum_{i=1}^{2}\left(\mathbf{P}^{(i)}, \operatorname{GRAD} \boldsymbol{\eta}^{(i)}\right)=\sum_{i=1}^{2}\left(\rho_{0}^{(i)} \mathbf{B}^{(i)}, \boldsymbol{\eta}^{(i)}\right) \\
& \quad+\sum_{i=1}^{2}\left(\overline{\mathbf{T}}^{(i)}, \boldsymbol{\eta}^{(i)}\right)_{\partial_{\sigma} \Omega^{(i)}}+\left(\mathbf{T}^{(1)}, \boldsymbol{\eta}^{(1)}-\boldsymbol{\eta}^{(2)}\right)_{\Gamma_{c}^{(1)}}
\end{aligned}
$$


for any admissible variations $\boldsymbol{\eta}^{(i)}: \Omega^{(i)} \rightarrow \mathbb{R}^{n_{\text {dim }}}$ such that $\boldsymbol{\eta}^{(i)}=\mathbf{0}$ on $\partial_{u} \Omega^{(i)}$.

\subsection{Linear momentum, angular momentum and total energy of the system}

The material form of the linear momentum $\mathbf{L}$ and angular momentum $\mathbf{J}$ of the system are given by:

$$
\begin{aligned}
\mathbf{L} & :=\sum_{i=1}^{2} \int_{\Omega^{(i)}} \rho_{0}^{(i)} \mathbf{V}^{(i)} d V \\
\mathbf{J} & :=\sum_{i=1}^{2} \int_{\Omega^{(i)}} \mathbf{x}^{(i)} \times \rho_{0}^{(i)} \mathbf{V}^{(i)} d V
\end{aligned}
$$

The total energy of a system $E$ can be additively split as:

$$
E:=K+W+\Pi^{e x t}
$$

where $K, W$ and $\Pi^{\text {ext }}$ are the kinetic energy, elastic strain energy and potential energy for the external loads, respectively, given by:
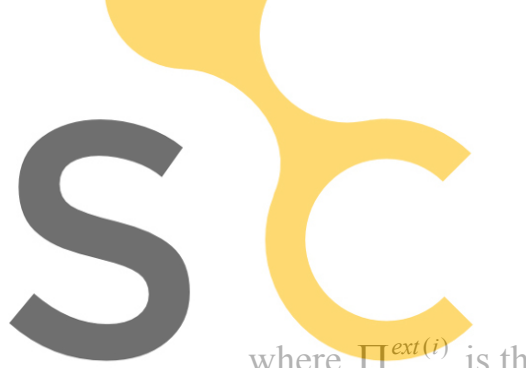

where $\Pi^{\text {ext }}$ (i) is the potential

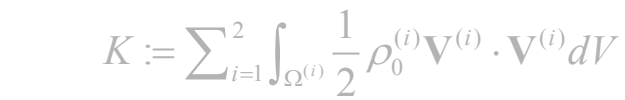

It can be shown $[8,9,21,34,49,51,52,54,55]$ that the linear momentum $\mathbb{L}$, angular Register for free at https/fwww.scipedia.com to download the version without the watermark

problem, characterized by zero body forces and zero natural boundary conditions, yielding zero potential energy for the external loads, i.e. $\Pi^{\text {ext }}=0$. The total energy $E$ is also conserved if the external loading is conservative. A typical case of conservative external loading is the case of gravitational body forces and constant prescribed nominal tractions.

\section{Finite element formulation of the continuum problem without frictional contact constraints}

Let us consider first the finite element discretization of quasi-static and dynamic continuum problem without frictional contact constraints. Using a standard finite element discretization, the material coordinates $\mathbf{X}^{(i)} \in \bar{\Omega}_{h}^{(i)}$, displacements $\mathbf{u}^{(i)}$ and material velocities $\mathbf{V}^{(i)}$ of body $\mathcal{B}^{(i)}$, take the form,

$$
\mathbf{X}^{(i)}:=\sum_{A=1}^{n_{\text {node }}^{(i)}} N_{A}(\boldsymbol{\xi}) \mathbf{X}_{A}^{(i)}, \quad \mathbf{u}^{(i)}:=\sum_{A=1}^{n_{\text {node }}^{(i)}} N_{A}(\boldsymbol{\xi}) \mathbf{u}_{A}^{(i)}, \quad \mathbf{V}^{(i)}:=\sum_{A=1}^{n_{\text {node }}^{(i)}} N_{A}(\boldsymbol{\xi}) \mathbf{V}_{A}^{(i)}
$$

where $\mathbf{x}^{(i)}:=\mathbf{X}^{(i)}+\mathbf{u}^{(i)}$ gives the current placement of the particle $\mathbf{X}^{(i)} \in \bar{\Omega}_{h}^{(i)}$ of body $\mathcal{B}^{(i)}$, $\mathbf{X}_{A}^{(i)} \in \bar{\Omega}_{h}^{(i)}, \mathbf{u}_{A}^{(i)}$ and $\mathbf{V}_{A}^{(i)}$ are the vectors of material coordinates, displacements and material 
velocities, respectively, of a node $A$ of the triangulation of body $\mathcal{B}^{(i)}, N_{A}(\xi): \square \mapsto \mathbb{R}$ is the interpolation shape function for node $A, \xi \in \square$ are the isoparametric coordinates defined in the unit domain $\square$, and $n_{\text {node }}^{(i)}$ is the number of nodes used in the triangulation of body $\mathcal{B}^{(i)}$.

Consider the time interval of interest $\mathbb{I}=[0, T]$ discretized into a series of nonoverlapping sub-intervals $\mathbb{I}=\bigcup\left[t_{n}, t_{n+1}\right]$. Using the standard convention, we denote by either $(\cdot)_{n+\alpha}$ the discrete approximations at time $t_{n+\alpha}$ of the continuum variable at time $t$.

\subsection{Quasi-static case}

The time discretization and finite element discretization of the variational form of the momentum balance equation for the quasi-static case yields the following expression for the residual force vector of a node $A$ of body $\mathcal{B}^{(i)}$ at time $n+1$,

$$
\mathbf{g}_{A}^{(i)}\left(\mathbf{u}_{n+1}^{(i)}\right):=\mathbf{f}_{A, n+1}^{\mathrm{int}(i)}\left(\mathbf{u}_{n+1}^{(i)}\right)-\mathbf{f}_{A, n+1}^{e x t(i)}=\mathbf{0}
$$

where $\mathbf{g}_{A}^{(i)}\left(\mathbf{u}_{n+1}^{(i)}\right), \mathbf{f}_{A, n+1}^{\mathrm{int}(i)}:=\mathbf{f}_{A, n+1}^{\mathrm{int}(i)}\left(\mathbf{u}_{n+1}^{(i)}\right)$ and $\mathbf{f}_{A, n+1}^{\text {ext }(i)}$ are the nodal vectors of residual forces, internal forces and external forces of node $A$ of body $\mathcal{B}^{(i)}$ at the time $n+1$, respectively.

Using an incremental iterative Newton-Raphson solution scheme, the linearization of the residual force vector given by (15) yields,
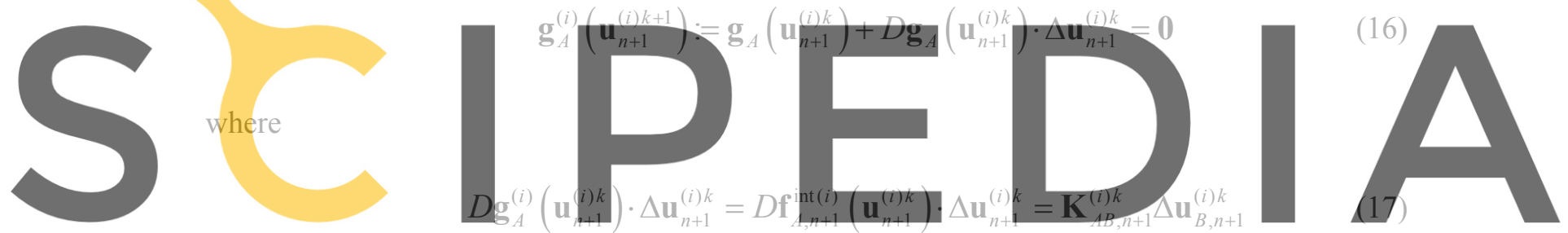

Register for free at https//www scipedia com to downhload the version without the watermark iteration $k$ of the time step $n+1$, and $\Delta \mathbf{u}_{B, n+1}^{(i) k}=\mathbf{u}_{B, n+1}^{(i) k+1}-\mathbf{u}_{B, n+1}^{(i) k}$.

\subsection{Dynamic case}

Using a mid-point time integration algorithm, the time discretization and finite element discretization of the variational form of the momentum balance equation for the dynamic case yields a discrete energy and momentum conserving time stepping algorithm, where the residual force vector of a node $A$ of body $\mathcal{B}^{(i)}$ at time $n+1$, takes the form [8,9,21,34,49,51,52,54,55],

$$
\mathbf{g}_{A}^{(i)}\left(\mathbf{u}_{n+1}^{(i)}\right):=\frac{1}{\Delta t} \mathbf{M}_{A B}^{(i)}\left(\mathbf{V}_{B, n+1}^{(i)}-\mathbf{V}_{B, n}^{(i)}\right)+\mathbf{f}_{A, n+1 / 2}^{\mathrm{int}(i)}\left(\mathbf{u}_{n+1}^{(i)}\right)-\mathbf{f}_{A, n+1 / 2}^{e x t(i)}=\mathbf{0}
$$

where $\mathbf{M}_{A B}^{(i)}$ is the mass matrix of nodes $A$ and $B$ of body $\mathcal{B}^{(i)}, \mathbf{V}_{B, n+1}^{(i)}$ is the vector of velocities of node $B$ of body $\mathcal{B}^{(i)}$ at the time $n+1$, given by,

$$
\mathbf{V}_{B, n+1}^{(i)}=2 \mathbf{V}_{B, n+1 / 2}^{(i)}-\mathbf{V}_{B, n}^{(i)}=\frac{2}{\Delta t}\left(\mathbf{u}_{B, n+1}^{(i)}-\mathbf{u}_{B, n}^{(i)}\right)-\mathbf{V}_{B, n}^{(i)}
$$


and $\mathbf{f}_{A, n+1 / 2}^{\mathrm{int}(i)}\left(\mathbf{u}_{n+1}^{(i)}\right)$ and $\mathbf{f}_{A, n+1 / 2}^{\text {ext }(i)}$ are the nodal vectors of internal forces and external forces of node $A$ of body $\mathcal{B}^{(i)}$ at the time $n+1 / 2$, respectively.

Using an incremental iterative Newton-Raphson solution scheme, the linearization of the discrete residual force vector given by (18) yields,

$$
\mathbf{g}_{A}^{(i)}\left(\mathbf{u}_{n+1}^{(i) k+1}\right):=\mathbf{g}_{A}^{(i)}\left(\mathbf{u}_{n+1}^{(i) k}\right)+D \mathbf{g}_{A}^{(i)}\left(\mathbf{u}_{n+1}^{(i) k}\right) \cdot \Delta \mathbf{u}_{n+1}^{(i) k}=\mathbf{0}
$$

where

$$
\begin{aligned}
D \mathbf{g}_{A}^{(i)}\left(\mathbf{u}_{n+1}^{(i) k}\right) \cdot \Delta \mathbf{u}_{n+1}^{(i) k} & =\frac{2}{\Delta t^{2}} \mathbf{M}_{A B}^{(i)} \Delta \mathbf{u}_{B, n+1}^{(i) k}+D \mathbf{f}_{A, n+1 / 2}^{\operatorname{int}(i) k}\left(\mathbf{u}_{n+1}^{(i) k}\right) \cdot \Delta \mathbf{u}_{n+1}^{(i) k} \\
& =\left(\frac{2}{\Delta t^{2}} \mathbf{M}_{A B}^{(i)}+\hat{\mathbf{K}}_{A B, n+1}^{(i) k}\right) \Delta \mathbf{u}_{B, n+1}^{(i) k}:=\mathbf{K}_{A B, n+1}^{(i) k} \Delta \mathbf{u}_{B, n+1}^{(i) k}
\end{aligned}
$$

where $k$ denotes the iteration number, $\mathbf{K}_{A B, n+1}^{(i) k}$ is the $A B$ component of the tangent stiffness matrix evaluated at the iteration $k$ of the time step $n+1$, and $\Delta \mathbf{u}_{B, n+1}^{(i) k}=\mathbf{u}_{B, n+1}^{(i) k+1}-\mathbf{u}_{B, n+1}^{(i) k}$.

The space semi-discrete versions $\mathbf{L}_{h}, \mathbf{J}_{h}$ and $E_{h}$ of the linear momentum, angular momentum and total energy, respectively, take the form [6-9,51]:
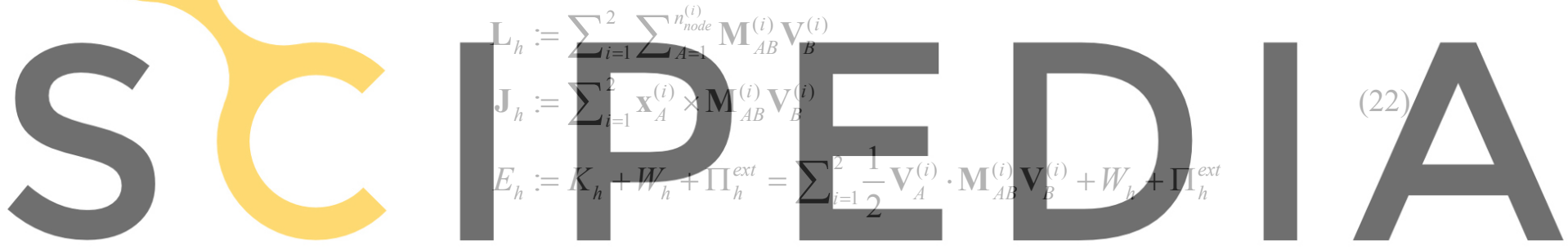

where Einstein's notation has been assumed for repeated indices $A$ and $B$.

Register for free at https c/lwww.scipedia.com to download the version without the watermark

discrete version of the linear momentum $\mathbb{L}_{h}$, angular momentum $\mathbb{J}_{h}$, and total energy $E_{h}$ are conserved for a homogeneous Neumann boundary problem, characterized by no imposed boundary displacements and zero external loading, zero body forces and zero natural boundary conditions, yielding zero semi-discrete external force vector, $\mathbf{f}_{A}^{\text {ext }(i)}=\mathbf{0}$, and zero semi discrete potential energy for the external loads, $\Pi_{h}^{\text {ext }}=0$. The discrete versión of the total energy $E_{h}$ is also conserved if the external loading is conservative.

\section{Direct elimination algorithm for contact problems}

\subsection{Introduction and notation}

Within the direct elimination algorithm for contact problems proposed in this work, the restrictions arising by the contact between the bodies are introduced through the direct elimination of the displacements of the slave nodes. From a computational implementation point of view, this direct elimination method is carried out through a number of transformations made on the global tangent operator. In order to conveniently visualize those transformations, let us introduce the following notation. 
Let us consider a generic slave node $s$ which is in contact with a given master element. Let us denote by $\left\{m_{1}, \ldots, m_{\text {mnod }}\right\}$ the set of master nodes of the master element which is in contact with the slave node $s$, where mnod is the number of nodes of the master element.

Furthermore, let us introduce the following notation for the set of nodes of the slave body which are connected to the slave node $s$, including both the nodes on the slave surface and the nodes in the interior domain of the slave body, and to the set of nodes of the master surface which are connected to the master nodes $\left\{m_{1}, \ldots, m_{\text {mnod }}\right\}$, including both the nodes of the master surface and the nodes in the interior domain of the master body.

Let us denote by $\left\{g s_{1}, \ldots, g s_{\text {gsnod }}\right\}$ the set of nodes of the slave body connected to the given slave node $s$, where gsnod is the number of nodes of this set, and let us denote by $\left\{g m_{1}, \ldots, g m_{\text {gmnod }}\right\}$ the set of nodes of the master body connected to the set of nodes $\left\{m_{1}, \ldots, m_{\text {mnod }}\right\}$, where gmnod is the number of nodes of this set.

Figure 1 shows the notation introduced for a typical slave node-master segment 2D contact problem using linear elements.

Linked to the notation introduced above for the slave and master nodes, let us introduce the following notation for the vector of displacements. Let us denote as $\mathbf{u}_{s}$ the vector of displacements of the slave node $s, \mathbf{u}_{m}$ the vector collecting the displacements of the set of nodes $\left\{m_{1}, \ldots, m_{m n o d}\right\}, \mathbf{u}_{g s}$ the vector collecting the displacements of the set of nodes $\left\{g s_{1}, \ldots, g s_{g s n o d}\right\}$, and $\mathbf{u}_{g m}$ the vector collecting the displacements of the set of nodes $\left\{g m_{1}, \ldots, g m_{\text {gmnod }}\right\}, \mathbf{u}_{g 1}$ the vector collecting the vector of displacements of the remaining set of nodes of body $\mathcal{B}^{(1)}$ and $\mathbf{u}_{g 2}$ the vector collecting the vector of displacements of the remaining nodes of body $\mathcal{B}^{(2)}$.

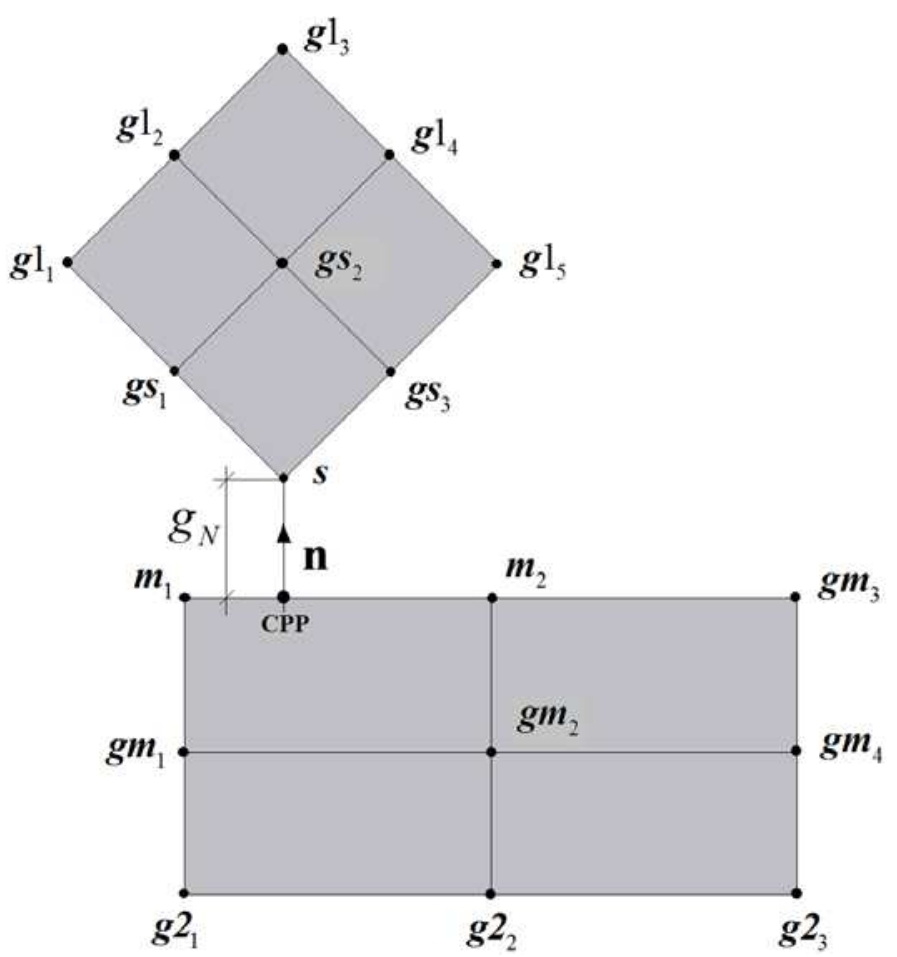

Figure 1. Definition and notation used for a slave node-to-master segment contact problem.

The vector position of an arbitrary point of a master surface element can be defined as, 


$$
\mathbf{x}=\sum_{i=1}^{n_{\text {mnod }}} N_{i}(\boldsymbol{\xi}) \mathbf{x}_{m i}=\sum_{i=1}^{n_{\text {mnod }}} \mathbf{N}_{i}(\boldsymbol{\xi}) \mathbf{x}_{m i}
$$

where $\xi \in \square$ are the isoparametric coordinates defined in the isoparametric unit domain $\square$, $N_{i}(\xi)$ are the interpolation shape functions of the nodes of the master element, $\mathbf{N}_{i}(\boldsymbol{\xi})=N_{i}(\boldsymbol{\xi}) \mathbf{1}$ is a diagonal matrix of shape functions.

The vector position of the closest-point-projection (CPP) of the slave node $s$ on the master element can be defined as,

$$
\mathbf{x}_{C P P}=\sum_{i=1}^{n_{\text {mood }}} N_{i}(\bar{\xi}) \mathbf{x}_{m i}=\sum_{i=1}^{n_{\text {mnod }}} \mathbf{N}_{i}(\bar{\xi}) \mathbf{x}_{m i}=\mathbf{N} \mathbf{x}_{m}
$$

where $\bar{\xi} \in \square$ are the isoparametric coordinates of the closest-point-projection defined in the isoparametric unit domain $\square$, and $\mathbf{N}=\left[\mathbf{N}_{1}(\bar{\xi}), \ldots, \mathbf{N}_{\text {mnod }}(\overline{\boldsymbol{\xi}})\right]$ is the matrix of nodal shape functions of the master nodes evaluated at $\bar{\xi} \in \square$.

Using the notation introduced above, the semi-discrete contact normal gap $g_{N}$ can be defined as,

$$
g_{N}:=\left(\mathbf{x}_{s}-\mathbf{x}_{C P P}\right) \cdot \mathbf{n}=\left(\mathbf{x}_{s}-\sum_{i=1}^{n_{\text {mmod }}} N_{i}(\bar{\xi}) \mathbf{x}_{m i}\right) \cdot \mathbf{n}=\left(\mathbf{x}_{s}-\mathbf{N} \mathbf{x}_{m}\right) \cdot \mathbf{n}
$$

where $\mathbf{x}_{s}$ is the current vector position of the slave node $s$ and $\mathbf{n}$ is the outward unit normal to the master element at the closest-point-projection of the slave node $s$.

\subsection{Contactless case}

Let us consider first a slave node $s$ which is not yet in contact with a master surface at time $n+1$. The residual force vectors can be written as:

$$
\begin{array}{r}
\mathbf{g}_{s}\left(\mathbf{u}_{s, n+1}, \mathbf{u}_{g s, n+1}\right)=\mathbf{0} \\
\mathbf{g}_{m}\left(\mathbf{u}_{m, n+1}, \mathbf{u}_{g m, n+1}\right)=\mathbf{0} \\
\mathbf{g}_{g s}\left(\mathbf{u}_{s, n+1}, \mathbf{u}_{g s, n+1}, \mathbf{u}_{g 1, n+1}\right)=\mathbf{0} \\
\mathbf{g}_{g m}\left(\mathbf{u}_{m, n+1}, \mathbf{u}_{g m, n+1}, \mathbf{u}_{g 2, n+1}\right)=\mathbf{0}
\end{array}
$$

Using an incremental iterative Newton-Raphson solution scheme, the linearization of the residuals (26) takes the form: 


$$
\begin{aligned}
& \mathbf{g}_{s}\left(\mathbf{u}_{s, n+1}^{k}, \mathbf{u}_{g s, n+1}^{k}\right)+D \mathbf{g}_{s}\left(\mathbf{u}_{s, n+1}^{k}, \mathbf{u}_{g s, n+1}^{k}\right) \cdot \Delta \mathbf{u}_{s, n+1}^{k}+D \mathbf{g}_{s}\left(\mathbf{u}_{s, n+1}^{k}, \mathbf{u}_{g s, n+1}^{k}\right) \cdot \Delta \mathbf{u}_{g s, n+1}^{k}=\mathbf{0} \\
& \mathbf{g}_{m}\left(\mathbf{u}_{m, n+1}^{k}, \mathbf{u}_{g m, n+1}^{k}\right)+D \mathbf{g}_{m}\left(\mathbf{u}_{m, n+1}^{k}, \mathbf{u}_{g m, n+1}^{k}\right) \cdot \Delta \mathbf{u}_{m, n+1}^{k}+D \mathbf{g}_{m}\left(\mathbf{u}_{m, n+1}^{k}, \mathbf{u}_{g m, n+1}^{k}\right) \cdot \Delta \mathbf{u}_{g m, n+1}^{k}=\mathbf{0} \\
& \mathbf{g}_{g s}\left(\mathbf{u}_{s, n+1}^{k}, \mathbf{u}_{g s, n+1}^{k}, \mathbf{u}_{g 1, n+1}^{k}\right)+D \mathbf{g}_{g s}\left(\mathbf{u}_{s, n+1}^{k}, \mathbf{u}_{g s, n+1}^{k}, \mathbf{u}_{g 1, n+1}^{k}\right) \cdot \Delta \mathbf{u}_{s, n+1}^{k} \\
& \quad+D \mathbf{g}_{g s}\left(\mathbf{u}_{s, n+1}^{k}, \mathbf{u}_{g s, n+1}^{k}, \mathbf{u}_{g 1, n+1}^{k}\right) \cdot \Delta \mathbf{u}_{g s, n+1}^{k}+D \mathbf{g}_{g s}\left(\mathbf{u}_{s, n+1}^{k}, \mathbf{u}_{g s, n+1}^{k}, \mathbf{u}_{g 1, n+1}^{k}\right) \cdot \Delta \mathbf{u}_{g 1, n+1}^{k}=\mathbf{0} \\
& \mathbf{g}_{g m}\left(\mathbf{u}_{m, n+1}^{k}, \mathbf{u}_{g m, n+1}^{k}, \mathbf{u}_{g 2, n+1}^{k}\right)+D \mathbf{g}_{g m}\left(\mathbf{u}_{m, n+1}^{k}, \mathbf{u}_{g m, n+1}^{k}, \mathbf{u}_{g 2, n+1}^{k}\right) \cdot \Delta \mathbf{u}_{m, n+1}^{k} \\
& \quad+D \mathbf{g}_{g m}\left(\mathbf{u}_{m, n+1}^{k}, \mathbf{u}_{g m, n+1}^{k}, \mathbf{u}_{g 2, n+1}^{k}\right) \cdot \Delta \mathbf{u}_{g m, n+1}^{k}+D \mathbf{g}_{g m}\left(\mathbf{u}_{m, n+1}^{k}, \mathbf{u}_{g m, n+1}^{k}, \mathbf{u}_{g 2, n+1}^{k}\right) \cdot \Delta \mathbf{u}_{g 2, n+1}^{k}=\mathbf{0}
\end{aligned}
$$

Introducing the following notation,

$$
\begin{aligned}
D \mathbf{g}_{m}\left(\mathbf{u}_{m, n+1}^{k}, \mathbf{u}_{g m, n+1}^{k}\right) \cdot \Delta \mathbf{u}_{m, n+1}^{k} & =\mathbf{K}_{m, m, n+1}^{k} \Delta \mathbf{u}_{m, n+1}^{k} \\
D \mathbf{g}_{m}\left(\mathbf{u}_{m, n+1}^{k}, \mathbf{u}_{g m, n+1}^{k}\right) \cdot \Delta \mathbf{u}_{g m, n+1}^{k} & =\mathbf{K}_{m, g m, n+1}^{k} \Delta \mathbf{u}_{g m, n+1}^{k} \\
D \mathbf{g}_{s}\left(\mathbf{u}_{s, n+1}^{k}, \mathbf{u}_{g s, n+1}^{k}\right) \cdot \Delta \mathbf{u}_{s, n+1}^{k} & =\mathbf{K}_{s, s, n+1}^{k} \Delta \mathbf{u}_{s, n+1}^{k} \\
D \mathbf{g}_{s}\left(\mathbf{u}_{s, n+1}^{k}, \mathbf{u}_{g s, n+1}^{k}\right) \cdot \Delta \mathbf{u}_{g s, n+1}^{k} & =\mathbf{K}_{s, g s, n+1}^{k} \Delta \mathbf{u}_{g s, n+1}^{k} \\
D \mathbf{g}_{g s}\left(\mathbf{u}_{s, n+1}^{k}, \mathbf{u}_{g s, n+1}^{k}, \mathbf{u}_{g 1, n+1}^{k}\right) \cdot \Delta \mathbf{u}_{s, n+1}^{k} & =\mathbf{K}_{g s, s, n+1}^{k} \Delta \mathbf{u}_{s, n+1}^{k} \\
D \mathbf{g}_{g s}\left(\mathbf{u}_{s, n+1}^{k}, \mathbf{u}_{g s, n+1}^{k}, \mathbf{u}_{g 1, n+1}^{k}\right) \cdot \Delta \mathbf{u}_{g s, n+1}^{k} & =\mathbf{K}_{g s, g s, n+1}^{k} \Delta \mathbf{u}_{g s, n+1}^{k} \\
D \mathbf{g}_{g s}\left(\mathbf{u}_{s, n+1}^{k}, \mathbf{u}_{g s, n+1}^{k}, \mathbf{u}_{g 1, n+1}^{k}\right) \cdot \Delta \mathbf{u}_{g 1, n+1}^{k} & =\mathbf{K}_{g s, g 1, n+1}^{k} \Delta \mathbf{u}_{g 1, n+1}^{k} \\
D \mathbf{g}_{g m}\left(\mathbf{u}_{m, n+1}^{k}, \mathbf{u}_{g m, n+1}^{k}, \mathbf{u}_{g 2, n+1}^{k}\right) \cdot \Delta \mathbf{u}_{m, n+1}^{k} & =\mathbf{K}_{g m, m, n+1}^{k} \Delta \mathbf{u}_{m, n+1}^{k} \\
D \mathbf{g}_{g m}\left(\mathbf{u}_{m, n+1}^{k}, \mathbf{u}_{g m, n+1}^{k}, \mathbf{u}_{g 2, n+1}^{k}\right) \cdot \Delta \mathbf{u}_{g m, n+1}^{k} & =\mathbf{K}_{g m, g m, n+1}^{k} \Delta \mathbf{u}_{g m, n+1}^{k} \\
D \mathbf{g}_{g m}\left(\mathbf{u}_{m, n+1}^{k}, \mathbf{u}_{g m, n+1}^{k}, \mathbf{u}_{g 2, n+1}^{k}\right) \cdot \Delta \mathbf{u}_{g 2, n+1}^{k} & =\mathbf{K}_{g m, g 2, n+1}^{k} \Delta \mathbf{u}_{g 2, n+1}^{k}
\end{aligned}
$$

yields the following linearized system of equations,

$$
\begin{gathered}
\mathbf{K}_{s, s, n+1}^{k} \Delta \mathbf{u}_{s, n+1}^{k}+\mathbf{K}_{s, g s, n+1}^{k} \Delta \mathbf{u}_{g s, n+1}^{k}=-\mathbf{g}_{s}\left(\mathbf{u}_{s, n+1}^{k}, \mathbf{u}_{g s, n+1}^{k}\right) \\
\mathbf{K}_{m, m, n+1}^{k} \Delta \mathbf{u}_{m, n+1}^{k}+\mathbf{K}_{m, g m, n+1}^{k} \Delta \mathbf{u}_{g m, n+1}^{k}=-\mathbf{g}_{m}\left(\mathbf{u}_{m, n+1}^{k}, \mathbf{u}_{g m, n+1}^{k}\right) \\
\mathbf{K}_{g s, s, n+1}^{k} \Delta \mathbf{u}_{s, n+1}^{k}+\mathbf{K}_{g s, g s, n+1}^{k} \Delta \mathbf{u}_{g s, n+1}^{k}+\mathbf{K}_{g s, g 1, n+1}^{k} \Delta \mathbf{u}_{g 1, n+1}^{k}=-\mathbf{g}_{g s}\left(\mathbf{u}_{s, n+1}^{k}, \mathbf{u}_{g s, n+1}^{k}, \mathbf{u}_{g 1, n+1}^{k}\right) \\
\mathbf{K}_{g m, m, n+1}^{k} \Delta \mathbf{u}_{m, n+1}^{k}+\mathbf{K}_{g m, g m, n+1}^{k} \Delta \mathbf{u}_{g m, n+1}^{k}+\mathbf{K}_{g m, g 2, n+1}^{k} \Delta \mathbf{u}_{g 2, n+1}^{k}=-\mathbf{g}_{g m}\left(\mathbf{u}_{m, n+1}^{k}, \mathbf{u}_{g m, n+1}^{k}, \mathbf{u}_{g 2, n+1}^{k}\right)
\end{gathered}
$$

where $\mathbf{K}_{s, s, n+1}^{k}$ and $\mathbf{K}_{s, g s, n+1}^{k}$ are the tangent stiffness blocks corresponding to row $s$ and columns $s$ and $g s$, respectively, $\mathbf{K}_{m, m, n+1}^{k}$ and $\mathbf{K}_{m, g m, n+1}^{k}$ are the tangent stiffness blocks corresponding to row $m$ and columns $m$ and $g m$, respectively, $\mathbf{K}_{g s, s, n+1}^{k}, \mathbf{K}_{g s, g s, n+1}^{k}$ and $\mathbf{K}_{g s, g 1, n+1}^{k}$ are the tangent stiffness blocks corresponding to row $g s$ and columns $s, g s$ and $g 1$, respectively, and $\mathbf{K}_{g m, m, n+1}^{k}, \mathbf{K}_{g m, g m, n+1}^{k}$ and $\mathbf{K}_{g m, g 2, n+1}^{k}$ are the tangent stiffness blocks corresponding to row $g m$ and columns $m, g m$ and $g 2$, respectively, all of them evaluated at the iteration $k$ of time $n+1$.

The resulting global linearized system of equations for the contactless case can be written in matrix form as, 


$$
\left[\begin{array}{cccccc}
\mathbf{K}_{s, s} & \mathbf{K}_{s, g s} & \mathbf{0} & \mathbf{0} & \mathbf{0} & \mathbf{0} \\
\mathbf{K}_{g s, s} & \mathbf{K}_{g s, g s} & \mathbf{K}_{g s, g 1} & \mathbf{0} & \mathbf{0} & \mathbf{0} \\
\mathbf{0} & \mathbf{K}_{g 1, g s} & \mathbf{K}_{g 1, g 1} & \mathbf{0} & \mathbf{0} & \mathbf{0} \\
\mathbf{0} & \mathbf{0} & \mathbf{0} & \mathbf{K}_{m, m} & \mathbf{K}_{m, g m} & \mathbf{0} \\
\mathbf{0} & \mathbf{0} & \mathbf{0} & \mathbf{K}_{g m, m} & \mathbf{K}_{g m, g m} & \mathbf{K}_{g m, g 2} \\
\mathbf{0} & \mathbf{0} & \mathbf{0} & \mathbf{0} & \mathbf{K}_{g 2, g m} & \mathbf{K}_{g 2, g 2}
\end{array}\right]_{n+1}^{k}\left[\begin{array}{c}
\Delta \mathbf{u}_{s} \\
\Delta \mathbf{u}_{g s} \\
\Delta \mathbf{u}_{g 1} \\
\Delta \mathbf{u}_{m} \\
\Delta \mathbf{u}_{g m} \\
\Delta \mathbf{u}_{g 2}
\end{array}\right]_{n+1}=-\left[\begin{array}{c}
\mathbf{g}_{s} \\
\mathbf{g}_{g s} \\
\mathbf{g}_{g 1} \\
\mathbf{g}_{m} \\
\mathbf{g}_{g m} \\
\mathbf{g}_{g 2}
\end{array}\right]_{n+1}^{k}
$$

\subsection{Full stick frictional contact case}

Once contact penetration is detected, the position of the slave node $s$ is subjected to the constraints arising from the full stick frictional contact condition. Note that for the full stick frictional case, once the slave node comes into contact with a master surface, the isoparametric coordinates of the closest-point-projection are time-independent, remaining constant in time while contact is active.

\subsubsection{Quasi-static case}

For a quasi-static case, the current position of the slave node $s$ is attached to the current position of the closest-point-projection on the master surface, which is constant in time, yielding the following expression:

$$
\mathbf{x}_{s, n+1}=\sum_{i=1}^{n_{\text {mood }}} N_{i}(\overline{\boldsymbol{\xi}}) \mathbf{x}_{m i, n+1}=\sum_{i=1}^{n_{\text {mood }}} \mathbf{N}_{i}(\overline{\boldsymbol{\xi}}) \mathbf{x}_{m i, n+1}=\mathbf{N} \mathbf{x}_{m, n+1}
$$

where $\bar{\xi} \in \square$ are the time-independent isoparametric coordinates of the closest-pointprojection.

\subsubsection{Dynamic case}

For the dynamic case, using a discrete linear momentum and energy conserving time integration scheme, the mid-point velocity of the slave node $s$ is matched to the mid-point velocity of its closest-point-projection, yielding the following expression [6]:

$$
\mathbf{v}_{s, n+1 / 2}=\sum_{i=1}^{n_{\text {mnod }}} N_{i}(\overline{\boldsymbol{\xi}}) \mathbf{v}_{m i, n+1 / 2}=\sum_{i=1}^{n_{\text {mood }}} \mathbf{N}_{i}(\overline{\boldsymbol{\xi}}) \mathbf{v}_{m i, n+1 / 2}=\mathbf{N} \mathbf{v}_{m, n+1 / 2}
$$

where the (time-independent) isoparametric coordinates of the closest-point-projection are computed at the mid-point configuration.

Using a mid-point rule time integration, equation (32) yields,

$$
\mathbf{x}_{s, n+1}=\mathbf{x}_{s, n}+\mathbf{N}\left(\mathbf{x}_{m, n+1}-\mathbf{x}_{m, n}\right), \quad \Delta \mathbf{u}_{s, n+1}=\mathbf{N} \Delta \mathbf{u}_{m, n+1}
$$

Note that, for the dynamic case, it is not possible to get an algorithm simultaneously satisfying discrete energy and angular momentum conservation [6]. The contact constraint (32) yields a discrete energy conservation algorithm, but the discrete angular momentum is not satisfied. Alternatively, imposing that the mid-point position of the slave node $s$ has to be equal 
to the mid-point position of its closest-point-projection, would yield a discrete momentum conservation algorithm, but then the discrete energy conservation would not be satisfied [6].

\subsubsection{Virtual contact work}

Let us denote as $\mathbf{f}_{s, n+\alpha}$ the discrete contact force acting on the slave node $s$ at time $n+\alpha$, and $\mathbf{f}_{m, n+\alpha}$ the vector collecting the discrete contact forces acting on the nodes of the master element at time $n+\alpha$, where $\alpha=1$ for the quasi-static case and $\alpha=1 / 2$ for the dynamic case. Applying the virtual work principle to the discrete contact force vectors $\mathbf{f}_{s, n+\alpha}$ and $\mathbf{f}_{m, n+\alpha}$ reads,

$$
\delta \mathbf{u}_{s} \cdot \mathbf{f}_{s, n+\alpha}+\delta \mathbf{u}_{m} \cdot \mathbf{f}_{m, n+\alpha}=0
$$

where $\delta \mathbf{u}_{s}$ and $\delta \mathbf{u}_{m}$ are virtual displacements of the slave and master element nodes, such that, taking into account that the isoparametric coordinates of the closest-point-projection remain constant, yields,

$$
\delta \mathbf{u}_{s}=\mathbf{N} \delta \mathbf{u}_{m}
$$

Substituting (35) into (34) yields,

$$
\mathbf{f}_{m, n+\alpha}=-\mathbf{N}^{T} \mathbf{f}_{s, n+\alpha}
$$

\subsubsection{Solution of the system of equations using a direct elimination method}

For a quasi-static or dynamic frictional contact problem, the discrete residual force vectors can be written as:

$$
\begin{array}{r}
\mathbf{g}_{s}\left(\mathbf{u}_{s, n+1}, \mathbf{u}_{g s, n+1}\right)-\mathbf{f}_{s, n+\alpha}=\mathbf{0} \\
\mathbf{g}_{m}\left(\mathbf{u}_{m, n+1}, \mathbf{u}_{g m, n+1}\right)-\mathbf{f}_{m, n+\alpha}=\mathbf{0} \\
\mathbf{g}_{g s}\left(\mathbf{u}_{s, n+1}, \mathbf{u}_{g s, n+1}, \mathbf{u}_{g 1, n+1}\right)=\mathbf{0} \\
\mathbf{g}_{g m}\left(\mathbf{u}_{m, n+1}, \mathbf{u}_{g m, n+1}, \mathbf{u}_{g 2, n+1}\right)=\mathbf{0}
\end{array}
$$

where $n+\alpha=n+1$ for a quasi-static case and $n+\alpha=n+1 / 2$ for a dynamic case.

From (37) , the discrete contact force vector acting on a slave node $s$ at time $n+\alpha$ can be written as,

$$
\mathbf{f}_{s, n+\alpha}=\mathbf{g}_{s}\left(\mathbf{u}_{s, n+1}, \mathbf{u}_{g s, n+1}\right)
$$

and substituting (38) into (36), and then (36) into (37) 2 , yields,

$$
\begin{aligned}
\mathbf{r}_{m}\left(\mathbf{u}_{m, n+1}, \mathbf{u}_{g m, n+1}, \mathbf{u}_{s, n+1}, \mathbf{u}_{g s, n+1}\right) & :=\mathbf{g}_{m}\left(\mathbf{u}_{m, n+1}, \mathbf{u}_{g m, n+1}\right)+\mathbf{N}^{T} \mathbf{g}_{s}\left(\mathbf{u}_{s, n+1}, \mathbf{u}_{g s, n+1}\right)=\mathbf{0} \\
\mathbf{r}_{g s}\left(\mathbf{u}_{s, n+1}, \mathbf{u}_{g s, n+1}, \mathbf{u}_{g 1, n+1}\right) & :=\mathbf{g}_{g s}\left(\mathbf{u}_{s, n+1}, \mathbf{u}_{g s, n+1}, \mathbf{u}_{g 1, n+1}\right)=\mathbf{0} \\
\mathbf{r}_{g m}\left(\mathbf{u}_{m, n+1}, \mathbf{u}_{g m, n+1}, \mathbf{u}_{g 2, n+1}\right) & :=\mathbf{g}_{g m}\left(\mathbf{u}_{m, n+1}, \mathbf{u}_{g m, n+1}, \mathbf{u}_{g 2, n+1}\right)=\mathbf{0}
\end{aligned}
$$


Using an incremental iterative Newton-Raphson solution scheme, taking into account that the closest-point-projection remains constant, the linearization of the above expressions takes the form:

$$
\begin{aligned}
& \mathbf{r}_{m}\left(\mathbf{u}_{m, n+1}^{k+1}, \mathbf{u}_{g m, n+1}^{k+1}, \mathbf{u}_{s, n+1}^{k+1}, \mathbf{u}_{g s, n+1}^{k+1}\right):=\mathbf{g}_{m}\left(\mathbf{u}_{m, n+1}^{k}, \mathbf{u}_{g m, n+1}^{k}\right)+\mathbf{N}^{T} \mathbf{g}_{s}\left(\mathbf{u}_{s, n+1}^{k}, \mathbf{u}_{g s, n+1}^{k}\right) \\
& \quad+D \mathbf{g}_{m}\left(\mathbf{u}_{m, n+1}^{k}, \mathbf{u}_{g m, n+1}^{k}\right) \cdot \Delta \mathbf{u}_{m, n+1}^{k}+D \mathbf{g}_{m}\left(\mathbf{u}_{m, n+1}^{k}, \mathbf{u}_{g m, n+1}^{k}\right) \cdot \Delta \mathbf{u}_{g m, n+1}^{k} \\
& \quad+\mathbf{N}^{T} D \mathbf{g}_{s}\left(\mathbf{u}_{s, n+1}^{k}, \mathbf{u}_{g s, n+1}^{k}\right) \cdot \Delta \mathbf{u}_{s, n+1}^{k}+\mathbf{N}^{T} D \mathbf{g}_{s}\left(\mathbf{u}_{s, n+1}^{k}, \mathbf{u}_{g s, n+1}^{k}\right) \cdot \Delta \mathbf{u}_{g s, n+1}^{k}=\mathbf{0} \\
& \mathbf{r}_{g s}\left(\mathbf{u}_{s, n+1}^{k+1}, \mathbf{u}_{g s, n+1}^{k+1}, \mathbf{u}_{g 1, n+1}^{k+1}\right)=\mathbf{g}_{g s}\left(\mathbf{u}_{s, n+1}^{k}, \mathbf{u}_{g s, n+1}^{k}, \mathbf{u}_{g 1, n+1}^{k}\right) \\
& \quad+D \mathbf{g}_{g s}\left(\mathbf{u}_{s, n+1}^{k}, \mathbf{u}_{g s, n+1}^{k}, \mathbf{u}_{g 1, n+1}^{k}\right) \cdot \Delta \mathbf{u}_{s, n+1}^{k}+D \mathbf{g}_{g s}\left(\mathbf{u}_{s, n+1}^{k}, \mathbf{u}_{g s, n+1}^{k}, \mathbf{u}_{g 1, n+1}^{k}\right) \cdot \Delta \mathbf{u}_{g s, n+1}^{k} \\
& \quad+D \mathbf{g}_{g s}\left(\mathbf{u}_{s, n+1}^{k}, \mathbf{u}_{g s, n+1}^{k}, \mathbf{u}_{g 1, n+1}^{k}\right) \cdot \Delta \mathbf{u}_{g 1, n+1}^{k}=\mathbf{0} \\
& \mathbf{r}_{g m}\left(\mathbf{u}_{m, n+1}^{k+1}, \mathbf{u}_{g m, n+1}^{k+1}, \mathbf{u}_{g 2, n+1}^{k+1}\right)=\mathbf{g}_{g m}\left(\mathbf{u}_{m, n+1}^{k}, \mathbf{u}_{g m, n+1}^{k}, \mathbf{u}_{g 2, n+1}^{k}\right) \\
& \quad+D \mathbf{g}_{g m}\left(\mathbf{u}_{m, n+1}^{k}, \mathbf{u}_{g m, n+1}^{k}, \mathbf{u}_{g 2, n+1}^{k}\right) \cdot \Delta \mathbf{u}_{m, n+1}^{k}+D \mathbf{g}_{g m}\left(\mathbf{u}_{m, n+1}^{k}, \mathbf{u}_{g m, n+1}^{k}, \mathbf{u}_{g 2, n+1}^{k}\right) \cdot \Delta \mathbf{u}_{g m, n+1}^{k} \\
& \quad+D \mathbf{g}_{g m}\left(\mathbf{u}_{m, n+1}^{k}, \mathbf{u}_{g m, n+1}^{k}, \mathbf{u}_{g 2, n+1}^{k}\right) \cdot \Delta \mathbf{u}_{g 2, n+1}^{k}=\mathbf{0}
\end{aligned}
$$

and using the notation introduced in (28), and $(33)_{2}$, yields the following linearized system of equations,

$$
\begin{aligned}
& \left(\mathbf{K}_{m, m, n+1}^{k}+\mathbf{N}^{T} \mathbf{K}_{s, s, n+1}^{k} \mathbf{N}\right) \Delta \mathbf{u}_{m, n+1}^{k}+\mathbf{K}_{m, g m, n+1}^{k} \Delta \mathbf{u}_{g m, n+1}^{k}+\mathbf{N}^{T} \mathbf{K}_{s, g s, n+1}^{k} \Delta \mathbf{u}_{g s, n+1}^{k} \\
& \quad=-\mathbf{g}_{m}\left(\mathbf{u}_{m, n+1}^{k}, \mathbf{u}_{g m, n+1}^{k}\right)-\mathbf{N}^{T} \mathbf{g}_{s}\left(\mathbf{u}_{s, n+1}^{k}, \mathbf{u}_{g s, n+1}^{k}\right) \\
& \mathbf{K}_{g s, s, n+1}^{k} \mathbf{N} \Delta \mathbf{u}_{m, n+1}^{k}+\mathbf{K}_{g s, g s, n+1}^{k} \Delta \mathbf{u}_{g s, n+1}^{k}+\mathbf{K}_{g s, g 1, n+1}^{k} \Delta \mathbf{u}_{g 1, n+1}^{k} \\
& \quad=-\mathbf{g}_{g s}\left(\mathbf{u}_{s, n+1}^{k}, \mathbf{u}_{g s, n+1}^{k}, \mathbf{u}_{g 1, n+1}^{k}\right) \\
& \mathbf{K}_{g m, m, n+1}^{k} \Delta \mathbf{u}_{m, n+1}^{k}+\mathbf{K}_{g m, g m, n+1}^{k} \Delta \mathbf{u}_{g m, n+1}^{k}+\mathbf{K}_{g m, g 2, n+1}^{k} \Delta \mathbf{u}_{g 2, n+1}^{k} \\
& \quad=-\mathbf{g}_{g m}\left(\mathbf{u}_{m, n+1}^{k}, \mathbf{u}_{g m, n+1}^{k}, \mathbf{u}_{g 2, n+1}^{k}\right)
\end{aligned}
$$

From an implementation point of view, starting from the global system of equations given in (30), the transformations of the global tangent stiffness matrix (GSM) and residual force vector (RFV) needed to implement the direct elimination method for the full stick frictional contact problem can be summarized in the following steps, which have to be carried out for each slave node $s$ :

Step 1 . Pre-multiply row $s$ of the GSM by the matrix $\mathbf{N}^{T}$.

Step 2. Add row $s$ to row $m$ of the GSM.

Step 3. Post-multiply column $s$ of the GSM by the matrix $\mathbf{N}$.

Step 4. Add column $s$ to column $m$ of the GSM.

Step 5. Set to zero matrix the row $s$, column $s$ of the GSM.

Step 6. Enter a diagonal matrix $\boldsymbol{\beta}_{n+1}^{k}=\beta_{n+1}^{k} \mathbf{1}$ in the row $s$, column $s$ of the GSM.

Step 7. Add the vector $\mathbf{N}^{T} \mathbf{g}_{s}\left(\mathbf{u}_{s, n+1}^{k}, \mathbf{u}_{g s, n+1}^{k}\right)$ to the row $s$ of the RFV. 
The diagonal matrix $\boldsymbol{\beta}=\boldsymbol{\beta} \mathbf{1}$, introduced in the row $s$, column $s$ of the GSM in order to avoid the ill-conditioning (zero terms in the main diagonal) of the GSM, is defined as,

$$
\boldsymbol{\beta}_{n+1}^{k}=\frac{1}{n_{\mathrm{dim}}}(\mathbf{1} \otimes \mathbf{1}): \mathbf{K}_{s, s, n+1}^{k}=\beta_{n+1}^{k} \mathbf{1}, \quad \boldsymbol{\beta}_{n+1}^{k}=\frac{1}{n_{\mathrm{dim}}} \mathbf{1}: \mathbf{K}_{s, s, n+1}^{k}=\frac{1}{n_{\mathrm{dim}}} \operatorname{tr}\left[\mathbf{K}_{s, s, n+1}^{k}\right]
$$

where $\operatorname{tr}[\cdot]$ denotes the trace operator and $n_{\mathrm{dim}}$ is the number of dimensions of the problem.

The resulting global linearized system of equations for the full stick frictional contact case can be written in matrix form as,

$$
\left[\begin{array}{cccccc}
\beta \mathbf{1} & \mathbf{0} & \mathbf{0} & \mathbf{0} & \mathbf{0} & \mathbf{0} \\
\mathbf{0} & \mathbf{K}_{g s, g s} & \mathbf{K}_{g s, g 1} & \mathbf{K}_{g s, s} \mathbf{N} & \mathbf{0} & \mathbf{0} \\
\mathbf{0} & \mathbf{K}_{g 1, g s} & \mathbf{K}_{g 1, g 1} & \mathbf{0} & \mathbf{0} & \mathbf{0} \\
\mathbf{0} & \mathbf{N}^{T} \mathbf{K}_{s, g s} & \mathbf{0} & \mathbf{K}_{m, m}+\mathbf{N}^{T} \mathbf{K}_{s, s} \mathbf{N} & \mathbf{K}_{m, g m} & \mathbf{0} \\
\mathbf{0} & \mathbf{0} & \mathbf{0} & \mathbf{K}_{g m, m} & \mathbf{K}_{g m, g m} & \mathbf{K}_{g m, g 2} \\
\mathbf{0} & \mathbf{0} & \mathbf{0} & \mathbf{0} & \mathbf{K}_{g 2, g m} & \mathbf{K}_{g 2, g 2}
\end{array}\right]_{n+1}^{k}\left[\begin{array}{c}
\Delta \mathbf{u}_{s} \\
\Delta \mathbf{u}_{g s} \\
\Delta \mathbf{u}_{g 1} \\
\Delta \mathbf{u}_{m} \\
\Delta \mathbf{u}_{g m} \\
\Delta \mathbf{u}_{g 2}
\end{array}\right]_{n+1}^{k}=-\left[\begin{array}{c}
\mathbf{0} \\
\mathbf{g}_{g s} \\
\mathbf{g}_{g 1} \\
\mathbf{g}_{m}+\mathbf{N}^{T} \mathbf{g}_{s} \\
\mathbf{g}_{g m} \\
\mathbf{g}_{g 2}
\end{array}\right]_{n+1}^{k}
$$

Remark 1. Note that block-symmetry of the resulting global tangent stiffness matrix for the full stick friction case is preserved.

\subsubsection{Update of slave and master displacements and contact status}

Once the resulting incremental iterative problem has been solved, the slave and master displacements are updated according to the following expressions.

For a quasi-static problem, the update of the master and slave displacements takes the form,

$$
\begin{aligned}
\mathbf{u}_{m, n+1}^{k+1} & =\mathbf{u}_{m, n+1}^{k}+\Delta \mathbf{u}_{m, n+1}^{k} \\
\mathbf{u}_{s, n+1}^{k+1} & =\mathbf{x}_{s, n+1}^{k+1}-\mathbf{X}_{s}=\mathbf{N} \mathbf{x}_{m, n+1}^{k+1}-\mathbf{X}_{s}=\mathbf{N} \mathbf{u}_{m, n+1}^{k+1}+\mathbf{N} \mathbf{X}_{m}-\mathbf{X}_{s}
\end{aligned}
$$

For a dynamic problem, using a discrete linear momentum and energy conserving algorithm, the update of the master and slave and displacements takes the form,

$$
\begin{aligned}
\mathbf{u}_{m, n+1}^{k+1} & =\mathbf{u}_{m, n+1}^{k}+\Delta \mathbf{u}_{m, n+1}^{k} \\
\mathbf{u}_{s, n+1}^{k+1} & =\mathbf{u}_{s, n+1}^{k}+\Delta \mathbf{u}_{s, n+1}^{k}=\mathbf{u}_{s, n+1}^{k}+\mathbf{N} \Delta \mathbf{u}_{m, n+1}^{k}
\end{aligned}
$$

For the dynamic case, once the displacements of the slave and master nodes have been updated, the nodal velocities of the slave and master nodes are updated using (19).

Once the slave and master nodes have been updated, the contact status at time $n+\alpha$ has to be verified, checking out if the contact is still active or not. The contact will be still active if the contact normal force $f_{N_{n+\alpha}}:=\mathbf{f}_{s, n+\alpha} \cdot \mathbf{n}_{n+\alpha}$ satisfies the following condition:

$$
f_{N_{n+\alpha}}:=\mathbf{f}_{s, n+\alpha} \cdot \mathbf{n}_{n+\alpha}=\mathbf{g}_{s}\left(\mathbf{u}_{s, n+1}, \mathbf{u}_{g s, n+1}\right) \cdot \mathbf{n}_{n+\alpha} \geq 0
$$


Otherwise, contact is lost and the contact status for the slave node $s$ has to be deactivated for the next time step.

\subsection{Frictionless contact case}

Once contact penetration is detected, the position of the slave node $S$ is subjected to the constraints arising from the frictionless contact condition. Note that, contrary to the full stick frictional case, for the frictionless case, the isoparametric coordinates of the closest-pointprojection are not constant in time. For the sake of concreteness, only the 3D frictionless quasistatic and dynamic cases will be presented, being straightforward to particularize the formulation for $2 \mathrm{D}$ cases.

\subsubsection{Quasi-static case}

For a quasi-static case, the current position of the slave node $s$ can be written in terms of the current position of the closest-point-projection, yielding the following expression:

$$
\mathbf{x}_{s, n+1}=\sum_{i=1}^{n_{\text {mnod }}} N_{i}\left(\overline{\boldsymbol{\xi}}_{n+1}\right) \mathbf{x}_{m i, n+1}=\sum_{i=1}^{n_{\text {mnod }}} \mathbf{N}_{i}\left(\overline{\boldsymbol{\xi}}_{n+1}\right) \mathbf{x}_{m i, n+1}=\mathbf{N}_{n+1} \mathbf{x}_{m, n+1}
$$

where $\overline{\boldsymbol{\xi}}_{n+1} \in \square$ are the time-dependent current isoparametric coordinates of the closest-pointprojection at time $n+1$.

Taking the variation of (47) yields,

$$
\begin{aligned}
\Delta \mathbf{x}_{s, n+1} & =\mathbf{N}_{n+1} \Delta \mathbf{x}_{m, n+1}+\Delta \mathbf{N}_{n+1} \mathbf{x}_{m, n+1} \\
& =\mathbf{N}_{n+1} \Delta \mathbf{x}_{m, n+1}+\mathbf{N}_{\xi, n+1} \mathbf{x}_{m, n+1} \Delta \bar{\xi}_{n+1}+\mathbf{N}_{\eta, n+1} \mathbf{x}_{m, n+1} \Delta \bar{\eta}_{n+1} \\
\Delta \mathbf{u}_{s, n+1} & =\mathbf{N}_{n+1} \Delta \mathbf{u}_{m, n+1}+\mathbf{N}_{\xi, n+1} \mathbf{x}_{m, n+1} \Delta \bar{\xi}_{n+1}+\mathbf{N}_{\eta, n+1} \mathbf{x}_{m, n+1} \Delta \bar{\eta}_{n+1}
\end{aligned}
$$

and taking into account that the covariant tangent vectors are given by $\boldsymbol{\tau}_{\xi, n+1}=\mathbf{N}_{\xi, n+1} \mathbf{x}_{m, n+1}$ and $\tau_{\eta, n+1}=\mathbf{N}_{\eta, n+1} \mathbf{x}_{m, n+1}$ (see Appendix 1), (48) 2 can be written as,

$$
\Delta \mathbf{u}_{s, n+1}=\mathbf{N}_{n+1} \Delta \mathbf{u}_{m, n+1}+\tau_{\xi, n+1} \Delta \bar{\xi}_{n+1}+\tau_{\eta, n+1} \Delta \bar{\eta}_{n+1}
$$

where $\Delta \bar{\xi}_{n+1}, \Delta \bar{\eta}_{n+1}$ are the contravariant components of the incremental slip of the closestpoint-projection on the covariant tangent basis $\tau_{\xi, n+1}, \tau_{\eta, n+1}$.

Let us denote as $\mathbf{f}_{s, n+1}$ the discrete contact force acting on the slave node $s$ at time $n+1$, and $\mathbf{f}_{m, n+1}$ the vector collecting the discrete contact forces acting on the nodes of the master element at time $n+1$. Applying the virtual work principle to the discrete contact force vectors $\mathbf{f}_{s, n+1}$ and $\mathbf{f}_{m, n+1}$ reads,

$$
\delta \mathbf{u}_{s} \cdot \mathbf{f}_{s, n+1}+\delta \mathbf{u}_{m} \cdot \mathbf{f}_{m, n+1}=0
$$

where $\delta \mathbf{u}_{s}$ and $\delta \mathbf{u}_{m}$ are the virtual displacements of the slave and master element nodes, such that, taking into account the variation of the isoparametric coordinates of the closest-pointprojection, satisfy the following expression (see Appendix 1), 


$$
\delta \mathbf{u}_{s}=\mathbf{N}_{n+1} \delta \mathbf{u}_{m}+\mathbf{N}_{\xi, n+1} \mathbf{x}_{m} \delta \bar{\xi}+\mathbf{N}_{\eta, n+1} \mathbf{x}_{m} \delta \bar{\eta}=\mathbf{N}_{n+1} \delta \mathbf{u}_{m}+\tau_{\xi, n+1} \delta \bar{\xi}+\tau_{\eta, n+1} \delta \bar{\eta}
$$

where $\tau_{\xi, n+1}$ and $\tau_{\eta, n+1}$ are the covariant tangent vectors to the isoparametric coordinates of the master surface at the closest-point-projection.

Substituting (51) into (50), and taking into account that $\tau_{\xi, n+1} \cdot \mathbf{f}_{s, n+1}=0$ and $\tau_{\eta, n+1} \cdot \mathbf{f}_{s, n+1}=0$, yields,

$$
\begin{aligned}
\mathbf{f}_{m, n+1} & =-\mathbf{N}_{n+1}^{T} \mathbf{f}_{s, n+1} \\
0 & =\boldsymbol{\tau}_{\xi, n+1} \cdot \mathbf{f}_{s, n+1} \\
0 & =\boldsymbol{\tau}_{\eta \cdot n+1} \cdot \mathbf{f}_{s, n+1}
\end{aligned}
$$

Remark 2. Note that the two last terms on the right hand side of (49) represent the tangent relative displacement of the slave node with respect to the closest-point-projection, here naturally expressed in terms of the variations of the contravariant components of the isoparametric coordinates $\Delta \bar{\xi}_{n+1}$ and $\Delta \bar{\eta}_{n+1}$ and the covariant tangent vectors to the isoparametric coordinates $\tau_{\xi, n+1}$ and $\tau_{\eta, n+1}$, evaluated at time $n+1$. Note that those tangent vectors span the tangent space at the closest-point-projection at time $n+1$, but they do not need to be orthonormal, not even orthogonal. Alternatively, the tangent space could be spanned by an orthonormal basis defined by orthogonal unit tangent vectors $\tau_{1, n+1}$ and $\tau_{2, n+1}$ at the closestpoint-projection at time $n+1$. Then, (49) can be alternatively expressed as,

$$
\Delta \mathbf{u}_{s, n+1}=\mathbf{N}_{n+1} \Delta \mathbf{u}_{m, n+1}+\Delta u_{s 1, n+1} \tau_{1, n+1}+\Delta u_{s 2, n+1} \tau_{2, n+1}
$$

where $\Delta u_{s 1, n+1}$ and $\Delta u_{s 2, n+1}$ are the components of the relative incremental slip displacement of the slave node $s$ with respect to the closest-point-projection, along the orthogonal unit tangent vectors $\tau_{1, n+1}$ and $\tau_{2, n+1}$, respectively.

Similarly, the constraints satisfied by the discrete contact force vectors $\mathbf{f}_{s, n+1}$ and $\mathbf{f}_{m, n+1}$ given by (52), can be written as,

$$
\begin{aligned}
\mathbf{f}_{m, n+1} & =-\mathbf{N}_{n+1}^{T} \mathbf{f}_{s, n+1} \\
0 & =\boldsymbol{\tau}_{1, n+1} \cdot \mathbf{f}_{s, n+1} \\
0 & =\boldsymbol{\tau}_{2, n+1} \cdot \mathbf{f}_{s, n+1}
\end{aligned}
$$

\subsubsection{Dynamic case}

For the dynamic case, a discrete linear momentum, angular momentum and energy conserving algorithm is obtained, imposing that the normal component of the slave node at the mid-point configuration has to be equal to the normal component of the velocity of its closest-pointprojection at the mid-point configuration, yielding,

$$
\mathbf{v}_{s, n+1 / 2} \cdot \mathbf{n}_{n+1 / 2}=\sum_{i=1}^{n_{\text {mood }}} N_{i}\left(\overline{\boldsymbol{\xi}}_{n+1 / 2}\right) \mathbf{v}_{m i, n+1 / 2} \cdot \mathbf{n}_{n+1 / 2}=\mathbf{N}_{n+1 / 2} \mathbf{v}_{m, n+1 / 2} \cdot \mathbf{n}_{n+1 / 2}
$$


where $\mathbf{n}_{n+1 / 2}$ is the outward unit normal to the closest-point-projection at the configuration at time $n+1 / 2$.

Using a mid-point time integration scheme, the normal velocity constraint given by (55) yields,

$$
\mathbf{x}_{s, n+1} \cdot \mathbf{n}_{n+1 / 2}=\left(\mathbf{x}_{s, n}+\mathbf{N}_{n+1 / 2}\left(\mathbf{x}_{m, n+1}-\mathbf{x}_{m, n}\right)\right) \cdot \mathbf{n}_{n+1 / 2}
$$

Then, the mid-point velocity of the slave node $s$ at time $n+1 / 2$, and the current placement and current increment of displacement of the slave node $s$ at time $n+1$ can be written as,

$$
\begin{aligned}
\mathbf{v}_{s, n+1 / 2} & =\mathbf{N}_{n+1 / 2} \mathbf{v}_{m, n+1 / 2}+v_{s 1, n+1 / 2} \tau_{1, n+1 / 2}+v_{s 2, n+1 / 2} \tau_{2, n+1 / 2} \\
\mathbf{x}_{s, n+1} & =\mathbf{x}_{s, n}+\mathbf{N}_{n+1 / 2}\left(\mathbf{x}_{m, n+1}-\mathbf{x}_{m, n}\right)+\Delta u_{s 1, n+1} \tau_{1, n+1 / 2}+\Delta u_{s 2, n+1} \tau_{2, n+1 / 2} \\
\Delta \mathbf{u}_{s, n+1} & =\mathbf{N}_{n+1 / 2} \Delta \mathbf{u}_{m, n+1}+\Delta u_{s 1, n+1} \tau_{1, n+1 / 2}+\Delta u_{s 2, n+1} \tau_{2, n+1 / 2}
\end{aligned}
$$

where $\tau_{1, n+1 / 2}$ and $\tau_{2, n+1 / 2}$ are orthogonal unit tangent vectors to the master surface at the midpoint closest-point-projection at time $n+1 / 2$, and $v_{s 1, n+1 / 2}$ and $v_{s 2, n+1 / 2}$ are the components of the relative mid-point slip velocity of the slave node $S$ along the unit tangent vectors $\tau_{1, n+1 / 2}$ and $\tau_{2, n+1 / 2}$, respectively, and $\Delta u_{s 1, n+1}$ and $\Delta u_{s 2, n+1}$ are the components of the relative incremental slip displacement of the slave node $s$ with respect to the closest-point-projection, along the orthogonal unit tangent vectors $\tau_{1, n+1 / 2}$ and $\tau_{2, n+1 / 2}$, respectively.

Let us denote as $\mathbf{f}_{s, n+1 / 2}$ the discrete contact force acting on the slave node $s$ at time $n+1 / 2$, and $\mathbf{f}_{m, n+1 / 2}$ the vector collecting the discrete contact forces acting on the nodes of the master element at time $n+1 / 2$. Applying the virtual work principle to the discrete contact force vectors $\mathbf{f}_{s, n+1 / 2}$ and $\mathbf{f}_{m, n+1 / 2}$ reads,

$$
\delta \mathbf{u}_{s} \cdot \mathbf{f}_{s, n+1 / 2}+\delta \mathbf{u}_{m} \cdot \mathbf{f}_{m, n+1 / 2}=0
$$

where $\delta \mathbf{u}_{s}$ and $\delta \mathbf{u}_{m}$ are virtual displacements of the slave node and master element nodes, respectively, such that,

$$
\delta \mathbf{u}_{s}=\mathbf{N}_{n+1 / 2} \delta \mathbf{u}_{m}+\delta \Delta u_{s 1} \tau_{1, n+1 / 2}+\delta \Delta u_{s 2} \tau_{2, n+1 / 2}
$$

Substituting (59) into (58), and taking into account that for a frictionless case, $\tau_{1 . n+1 / 2} \cdot \mathbf{f}_{s, n+1 / 2}=0$ and $\tau_{2, n+1 / 2} \cdot \mathbf{f}_{s, n+1 / 2}=0$, yields,

$$
\begin{aligned}
\mathbf{f}_{m, n+1 / 2} & =-\mathbf{N}_{n+1 / 2}^{T} \mathbf{f}_{s, n+1 / 2} \\
0 & =\tau_{1, n+1 / 2} \cdot \mathbf{f}_{s, n+1 / 2} \\
0 & =\tau_{2, n+1 / 2} \cdot \mathbf{f}_{s, n+1 / 2}
\end{aligned}
$$

\subsubsection{Solution of the system of equations using a direct elimination method}


For either a quasi-static or dynamic frictional contact problem, the discrete system of equations can be written as:

$$
\begin{aligned}
\mathbf{g}_{s}\left(\mathbf{u}_{s, n+1}, \mathbf{u}_{g s, n+1}\right)-\mathbf{f}_{s, n+\alpha} & =\mathbf{0} \\
\mathbf{g}_{m}\left(\mathbf{u}_{m, n+1}, \mathbf{u}_{g m, n+1}\right)-\mathbf{f}_{m, n+\alpha} & =\mathbf{0} \\
\mathbf{g}_{g s}\left(\mathbf{u}_{s, n+1}, \mathbf{u}_{g s, n+1}, \mathbf{u}_{g 1, n+1}\right) & =\mathbf{0} \\
\mathbf{g}_{g m}\left(\mathbf{u}_{m, n+1}, \mathbf{u}_{g m, n+1}, \mathbf{u}_{g 2, n+1}\right) & =\mathbf{0} \\
\tau_{1, n+\alpha} \cdot \mathbf{f}_{s, n+\alpha} & =0 \\
\boldsymbol{\tau}_{2, n+\alpha} \cdot \mathbf{f}_{s, n+\alpha} & =0
\end{aligned}
$$

where $n+\alpha=n+1$ for the quasi-static case, and $n+\alpha=n+1 / 2$ for the dynamic case.

From $(61)_{1}$, the discrete contact force vector acting on a slave node $s$ at time $n+\alpha$ can be written as,

$$
\mathbf{f}_{s, n+\alpha}=\mathbf{g}_{s}\left(\mathbf{u}_{s, n+1}, \mathbf{u}_{g s, n+1}\right)
$$

Substituting (62) into (60) $)_{1}$, and then (60) 1 into (61), yields,

$$
\begin{aligned}
\mathbf{r}_{m}\left(\mathbf{u}_{m, n+1}, \mathbf{u}_{g m, n+1}, \mathbf{u}_{s, n+1}, \mathbf{u}_{g s, n+1}\right) & :=\mathbf{g}_{m}\left(\mathbf{u}_{m, n+1}, \mathbf{u}_{g m, n+1}\right)+\mathbf{N}_{n+\alpha}^{T} \mathbf{g}_{s}\left(\mathbf{u}_{s, n+1}, \mathbf{u}_{g s, n+1}\right)=\mathbf{0} \\
\mathbf{r}_{g s}\left(\mathbf{u}_{s, n+1}, \mathbf{u}_{g s, n+1}, \mathbf{u}_{g 1, n+1}\right) & :=\mathbf{g}_{g s}\left(\mathbf{u}_{s, n+1}, \mathbf{u}_{g s, n+1}, \mathbf{u}_{g 1, n+1}\right)=\mathbf{0} \\
\mathbf{r}_{g m}\left(\mathbf{u}_{m, n+1}, \mathbf{u}_{g m, n+1}, \mathbf{u}_{g 2, n+1}\right) & :=\mathbf{g}_{g m}\left(\mathbf{u}_{m, n+1}, \mathbf{u}_{g m, n+1}, \mathbf{u}_{g 2, n+1}\right)=\mathbf{0} \\
r_{\tau_{1}}\left(\mathbf{u}_{s, n+1}, \mathbf{u}_{g s, n+1}\right) & :=\tau_{1, n+\alpha} \cdot \mathbf{g}_{s}\left(\mathbf{u}_{s, n+1}, \mathbf{u}_{g s, n+1}\right)=0 \\
r_{\tau_{2}}\left(\mathbf{u}_{s, n+1}, \mathbf{u}_{g s, n+1}\right) & :=\boldsymbol{\tau}_{2, n+\alpha} \cdot \mathbf{g}_{s}\left(\mathbf{u}_{s, n+1}, \mathbf{u}_{g s, n+1}\right)=0
\end{aligned}
$$

Using an incremental iterative Newton-Raphson solution scheme, taking into account the variation of the closest-point-projection, the linearization of the above expressions takes the form: 


$$
\begin{aligned}
\mathbf{r}_{m} & \left(\mathbf{u}_{m, n+1}^{k+1}, \mathbf{u}_{g m, n+1}^{k+1}, \mathbf{u}_{s, n+1}^{k+1}, \mathbf{u}_{g s, n+1}^{k+1}\right):=\mathbf{g}_{m}\left(\mathbf{u}_{m, n+1}^{k}, \mathbf{u}_{g m, n+1}^{k}\right)+\mathbf{N}_{n+\alpha}^{T k} \mathbf{g}_{s}\left(\mathbf{u}_{s, n+1}^{k}, \mathbf{u}_{g s, n+1}^{k}\right) \\
& +D \mathbf{g}_{m}\left(\mathbf{u}_{m, n+1}^{k}, \mathbf{u}_{g m, n+1}^{k}\right) \cdot \Delta \mathbf{u}_{m, n+1}^{k}+D \mathbf{g}_{m}\left(\mathbf{u}_{m, n+1}^{k}, \mathbf{u}_{g m, n+1}^{k}\right) \cdot \Delta \mathbf{u}_{g m, n+1}^{k} \\
& +\mathbf{N}_{n+\alpha}^{T k} D \mathbf{g}_{s}\left(\mathbf{u}_{s, n+1}^{k}, \mathbf{u}_{g s, n+1}^{k}\right) \cdot \Delta \mathbf{u}_{s, n+1}^{k}+\mathbf{N}_{n+\alpha}^{T k} D \mathbf{g}_{s}\left(\mathbf{u}_{s, n+1}^{k}, \mathbf{u}_{g s, n+1}^{k}\right) \cdot \Delta \mathbf{u}_{g s, n+1}^{k} \\
& +\Delta \mathbf{N}_{n+\alpha}^{T k} \mathbf{g}_{s}\left(\mathbf{u}_{s, n+1}^{k}, \mathbf{u}_{g s, n+1}^{k}\right)=\mathbf{0} \\
\mathbf{r}_{g s} & \left(\mathbf{u}_{s, n+1}^{k+1}, \mathbf{u}_{g s, n+1}^{k+1}, \mathbf{u}_{g 1, n+1}^{k+1}\right):=\mathbf{g}_{g s}\left(\mathbf{u}_{s, n+1}^{k}, \mathbf{u}_{g s, n+1}^{k}, \mathbf{u}_{g 1, n+1}^{k}\right) \\
& +D \mathbf{g}_{g s}\left(\mathbf{u}_{s, n+1}^{k}, \mathbf{u}_{g s, n+1}^{k}, \mathbf{u}_{g 1, n+1}^{k}\right) \cdot \Delta \mathbf{u}_{s, n+1}^{k}+D \mathbf{g}_{g s}\left(\mathbf{u}_{s, n+1}^{k}, \mathbf{u}_{g s, n+1}^{k}, \mathbf{u}_{g 1, n+1}^{k}\right) \cdot \Delta \mathbf{u}_{g s, n+1}^{k} \\
& +D \mathbf{g}_{g s}\left(\mathbf{u}_{s, n+1}^{k}, \mathbf{u}_{g s, n+1}^{k}, \mathbf{u}_{g 1, n+1}^{k}\right) \cdot \Delta \mathbf{u}_{g 1, n+1}^{k}=\mathbf{0} \\
\mathbf{r}_{g m} & \left(\mathbf{u}_{m, n+1}^{k+1}, \mathbf{u}_{g m, n+1}^{k+1}, \mathbf{u}_{g 2, n+1}^{k+1}\right):=\mathbf{g}_{g m}\left(\mathbf{u}_{m, n+1}^{k}, \mathbf{u}_{g m, n+1}^{k}, \mathbf{u}_{g 2, n+1}^{k}\right) \\
& +D \mathbf{g}_{g m}\left(\mathbf{u}_{m, n+1}^{k}, \mathbf{u}_{g m, n+1}^{k}, \mathbf{u}_{g 2, n+1}^{k}\right) \cdot \Delta \mathbf{u}_{m, n+1}^{k}+D \mathbf{g}_{g m}\left(\mathbf{u}_{m, n+1}^{k}, \mathbf{u}_{g m, n+1}^{k}, \mathbf{u}_{g 2, n+1}^{k}\right) \cdot \Delta \mathbf{u}_{g m, n+1}^{k} \\
& +D \mathbf{g}_{g m}\left(\mathbf{u}_{m, n+1}^{k}, \mathbf{u}_{g m, n+1}^{k}, \mathbf{u}_{g 2, n+1}^{k}\right) \cdot \Delta \mathbf{u}_{g 2, n+1}^{k}=\mathbf{0} \\
r_{\tau_{1}} & \left(\mathbf{u}_{s, n+1}^{k+1}, \mathbf{u}_{g s, n+1}^{k+1}\right):=\boldsymbol{\tau}_{1, n+\alpha}^{k} \cdot \mathbf{g}_{s}\left(\mathbf{u}_{s, n+1}^{k}, \mathbf{u}_{g s, n+1}^{k}\right) \\
& +\tau_{1, n+\alpha}^{k} D \mathbf{g}_{s}\left(\mathbf{u}_{s, n+1}^{k}, \mathbf{u}_{g s, n+1}^{k}\right) \cdot \Delta \mathbf{u}_{s, n+1}^{k}+\tau_{1, n+\alpha}^{k} D \mathbf{g}_{s}\left(\mathbf{u}_{s, n+1}^{k}, \mathbf{u}_{g s, n+1}^{k}\right) \cdot \Delta \mathbf{u}_{g s, n+1}^{k} \\
& +\mathbf{g}_{s}\left(\mathbf{u}_{s, n+1}^{k}, \mathbf{u}_{g s, n+1}^{k}\right) \cdot \Delta \tau_{1, n+\alpha}^{k}=0 \\
r_{\tau_{2}} & \left(\mathbf{u}_{s, n+1}^{k+1}, \mathbf{u}_{g s, n+1}^{k+1}\right):=\tau_{2, n+\alpha}^{k} \cdot \mathbf{g}_{s}\left(\mathbf{u}_{s, n+1}^{k}, \mathbf{u}_{g s, n+1}^{k}\right) \\
& +\boldsymbol{\tau}_{2, n+\alpha}^{k} D \mathbf{g}_{s}\left(\mathbf{u}_{s, n+1}^{k}, \mathbf{u}_{g s, n+1}^{k}\right) \cdot \Delta \mathbf{u}_{s, n+1}^{k}+\boldsymbol{\tau}_{2, n+\alpha}^{k} D \mathbf{g}_{s}\left(\mathbf{u}_{s, n+1}^{k}, \mathbf{u}_{g s, n+1}^{k}\right) \cdot \Delta \mathbf{u}_{g s, n+1}^{k} \\
& +\mathbf{g}_{s}\left(\mathbf{u}_{s, n+1}^{k}, \mathbf{u}_{g s, n+1}^{k}\right) \cdot \Delta \tau_{2, n+\alpha}^{k}=0
\end{aligned}
$$

where the variation of $\mathbf{N}_{n+\alpha}^{T k}$ and the variations of the orthogonal unit tangent vectors $\tau_{1, n+\alpha}^{k}$ and $\tau_{2, n+\alpha}^{k}$ (see Appendix 1) take the form,

$$
\begin{aligned}
\Delta \mathbf{N}_{n+\alpha}^{T k}= & \alpha \mathbf{N}_{\xi, n+\alpha}^{T k} \Delta \bar{\xi}_{n+1}^{k}+\alpha \mathbf{N}_{\eta, n+\alpha}^{T k} \Delta \bar{\eta}_{n+1}^{k} \\
= & \alpha \mathbf{N}_{\xi, n+\alpha}^{T k}\left(m_{1, n+\alpha}^{\xi k} \Delta u_{s 1, n+1}^{k}+m_{2, n+\alpha}^{\xi k} \Delta u_{s 2, n+1}^{k}\right) \\
& +\alpha \mathbf{N}_{\eta, n+\alpha}^{T k}\left(m_{1, n+\alpha}^{\eta k} \Delta u_{s 1, n+1}^{k}+m_{2, n+\alpha}^{\eta k} \Delta u_{s 2, n+1}^{k}\right) \\
= & \alpha\left(\mathbf{N}_{\xi, n+\alpha}^{T k} m_{1, n+\alpha}^{\xi k}+\mathbf{N}_{\eta, n+\alpha}^{T k} m_{1, n+\alpha}^{\eta k}\right) \Delta u_{s 1, n+1}^{k} \\
& +\alpha\left(\mathbf{N}_{\xi, n+\alpha}^{T k} m_{2, n+\alpha}^{\xi k}+\mathbf{N}_{\eta, n+\alpha}^{T k} m_{2, n+\alpha}^{\eta k}\right) \Delta u_{s 2, n+1}^{k} \\
\Delta \tau_{1, n+\alpha}^{k}= & \alpha\left(\mathbf{A}_{1, n+\alpha}^{k} \Delta \mathbf{u}_{m, n+1}^{k}+\overline{\mathbf{A}}_{1, n+\alpha}^{k} \Delta \mathbf{u}_{s, n+1}^{\prime k}\right) \\
\Delta \tau_{2, n+\alpha}^{k}= & \alpha\left(\mathbf{A}_{2, n+\alpha}^{k} \Delta \mathbf{u}_{m, n+1}^{k}+\overline{\mathbf{A}}_{2, n+\alpha}^{k} \Delta \mathbf{u}_{s, n+1}^{\prime k}\right)
\end{aligned}
$$

Using, either (53), for the quasi-static case, or $(57)_{3}$, for the dynamic case, and substituting (28) and (65) into (64), the discrete system of linearized equations takes the form, 


$$
\begin{aligned}
& \left(\mathbf{K}_{m, m, n+1}^{k}+\mathbf{N}_{n+\alpha}^{T k} \mathbf{K}_{s, s, n+1}^{k} \mathbf{N}_{n+\alpha}^{k}\right) \Delta \mathbf{u}_{m, n+1}^{k}+\mathbf{K}_{m, g m, n+1}^{k} \Delta \mathbf{u}_{g m, n+1}^{k}+\mathbf{N}_{n+\alpha}^{T k} \mathbf{K}_{s, g s, n+1}^{k} \Delta \mathbf{u}_{g s, n+1}^{k} \\
& +\mathbf{N}_{n+\alpha}^{T k} \mathbf{K}_{s, s, n+1}^{k}\left(\Delta u_{s 1, n+1}^{k} \tau_{1, n+\alpha}^{k}+\Delta u_{s 2, n+1}^{k} \tau_{2, n+\alpha}^{k}\right) \\
& +\alpha\left(\mathbf{N}_{\xi, n+\alpha}^{T k} m_{1, n+\alpha}^{\xi k}+\mathbf{N}_{\eta, n+\alpha}^{T k} m_{1, n+\alpha}^{\eta k}\right) \mathbf{g}_{s}\left(\mathbf{u}_{s, n+1}^{k}, \mathbf{u}_{g s, n+1}^{k}\right) \Delta u_{s 1, n+1}^{k} \\
& +\alpha\left(\mathbf{N}_{\xi, n+\alpha}^{T k} m_{2, n+\alpha}^{\xi k}+\mathbf{N}_{\eta, n+\alpha}^{T k} m_{2, n+\alpha}^{\eta k}\right) \mathbf{g}_{s}\left(\mathbf{u}_{s, n+1}^{k}, \mathbf{u}_{g s, n+1}^{k}\right) \Delta u_{s 2, n+1}^{k} \\
& =-\mathbf{g}_{m}\left(\mathbf{u}_{m, n+1}^{k}, \mathbf{u}_{g m, n+1}^{k}\right)-\mathbf{N}_{n+\alpha}^{T k} \mathbf{g}_{s}\left(\mathbf{u}_{s, n+1}^{k}, \mathbf{u}_{g s, n+1}^{k}\right) \\
& \mathbf{K}_{g s, s, n+1}^{k} \mathbf{N}_{n+\alpha}^{k} \Delta \mathbf{u}_{m, n+1}^{k}+\mathbf{K}_{g s, g s, n+1}^{k} \Delta \mathbf{u}_{g s, n+1}^{k}+\mathbf{K}_{g s, g 1, n+1}^{k} \Delta \mathbf{u}_{g 1, n+1}^{k} \\
& +\mathbf{K}_{g s, s, n+1}^{k}\left(\Delta u_{s 1, n+1}^{k} \tau_{1, n+\alpha}^{k}+\Delta u_{s 2, n+1}^{k} \tau_{2, n+\alpha}^{k}\right) \\
& =-\mathbf{g}_{g s}\left(\mathbf{u}_{s, n+1}^{k}, \mathbf{u}_{g s, n+1}^{k}, \mathbf{u}_{g 1, n+1}^{k}\right) \\
& \mathbf{K}_{g m, m, n+1}^{k} \Delta \mathbf{u}_{m, n+1}^{k}+\mathbf{K}_{g m, g m, n+1}^{k} \Delta \mathbf{u}_{g m, n+1}^{k}+\mathbf{K}_{g m, g 2, n+1}^{k} \Delta \mathbf{u}_{g 2, n+1}^{k} \\
& =-\mathbf{g}_{g m}\left(\mathbf{u}_{m, n+1}^{k}, \mathbf{u}_{g m, n+1}^{k}, \mathbf{u}_{g 2, n+1}^{k}\right) \\
& \left(\boldsymbol{\tau}_{1, n+\alpha}^{k} \mathbf{K}_{s, s, n+1}^{k} \mathbf{N}_{n+\alpha}^{k}+\alpha \mathbf{g}_{s}\left(\mathbf{u}_{s, n+1}^{k}, \mathbf{u}_{g s, n+1}^{k}\right) \cdot \mathbf{A}_{1, n+\alpha}^{k}\right) \Delta \mathbf{u}_{m, n+1}^{k} \\
& +\tau_{1, n+\alpha}^{k} \cdot \mathbf{K}_{s, g s, n+1}^{k} \Delta \mathbf{u}_{g s, n+1}^{k}+\tau_{1, n+\alpha}^{k} \cdot \mathbf{K}_{s, s, n+1}^{k}\left(\Delta u_{s 1, n+1}^{k} \tau_{1, n+\alpha}^{k}+\Delta u_{s 2, n+1}^{k} \tau_{2, n+\alpha}^{k}\right) \\
& +\alpha \mathbf{g}_{s}\left(\mathbf{u}_{s, n+1}^{k}, \mathbf{u}_{g s, n+1}^{k}\right) \cdot \overline{\mathbf{A}}_{1, n+\alpha}^{k} \Delta \mathbf{u}_{s, n+1}^{\prime k} \\
& =-\tau_{1, n+\alpha}^{k} \cdot \mathbf{g}_{s}\left(\mathbf{u}_{s, n+1}^{k}, \mathbf{u}_{g s, n+1}^{k}\right) \\
& \left(\tau_{2, n+\alpha}^{k} \mathbf{K}_{s, s, n+1}^{k} \mathbf{N}_{n+\alpha}^{k}+\alpha \mathbf{g}_{s}\left(\mathbf{u}_{s, n+1}^{k}, \mathbf{u}_{g s, n+1}^{k}\right) \mathbf{A}_{2, n+\alpha}^{k}\right) \Delta \mathbf{u}_{m, n+1}^{k} \\
& +\boldsymbol{\tau}_{2, n+\alpha}^{k} \cdot \mathbf{K}_{s, g s, n+1}^{k} \Delta \mathbf{u}_{g s, n+1}^{k}+\boldsymbol{\tau}_{2, n+\alpha}^{k} \cdot \mathbf{K}_{s, s, n+1}^{k}\left(\Delta u_{s 1, n+1}^{k} \tau_{1, n+\alpha}^{k}+\Delta u_{s 2, n+1}^{k} \tau_{2, n+\alpha}^{k}\right) \\
& +\alpha \mathbf{g}_{s}\left(\mathbf{u}_{s, n+1}^{k}, \mathbf{u}_{g s, n+1}^{k}\right) \overline{\mathbf{A}}_{2, n+\alpha}^{k} \Delta \mathbf{u}_{s, n+1}^{\prime k} \\
& =-\tau_{2, n+\alpha}^{k} \cdot \mathbf{g}_{s}\left(\mathbf{u}_{s, n+1}^{k}, \mathbf{u}_{g s, n+1}^{k}\right)
\end{aligned}
$$

From an implementation point of view, starting from the global system of linearized equations given in (30), the transformations of the global tangent stiffness matrix (GSM) and residual force vector (RFV) needed to implement the direct elimination method for the frictionless contact problem can be summarized in the following steps which have to be carried out for each slave node $s$ :

Step 1. Add to row $m$, column $m$ of the GSM, the matrix $\mathbf{N}_{n+\alpha}^{T k} \mathbf{K}_{s, s, n+1}^{k} \mathbf{N}_{n+\alpha}^{k}$.

Step 2. Add to row $m$, column $g s$ of the GSM, the matrix $\mathbf{N}_{n+\alpha}^{T k} \mathbf{K}_{s, b s}^{k}$

Step 3. Add to row $m$, column $s$ of the GSM, the matrix $\mathbf{N}_{n+\alpha}^{T k} \mathbf{K}_{s, s, n+1}^{k} \mathbf{H}_{n+\alpha}^{k}+\overline{\overline{\mathbf{A}}}_{n+\alpha}^{k}$.

Step 4. Add to row $g s$, column $m$ of the GSM, the matrix $\mathbf{K}_{g s, s, n+1}^{k} \mathbf{N}_{n+\alpha}^{k}$.

Step 5. Add to row $g s$, column $s$ of the GSM, the matrix $\mathbf{K}_{g s, s, n+1}^{k} \mathbf{H}_{n+\alpha}^{k}$.

Step 6. Replace row $s$, column $m$ of the GSM by the matrix

$\mathbf{H}_{n+\alpha}^{T k} \mathbf{K}_{s, s, n+1}^{k} \mathbf{N}_{n+\alpha}^{k}+\mathbf{A}_{n+\alpha}^{T k}$.

Step 7. Add to row $s$, column $g s$ of the GSM, the matrix $\mathbf{H}_{n+\alpha}^{T k} \mathbf{K}_{s, g s, n+1}^{k}$.

Step 8. Replace row $s$, column $s$ of the GSM by the matrix

$\mathbf{H}_{n+\alpha}^{T k} \mathbf{K}_{s, s, n+1}^{k} \mathbf{H}_{n+\alpha}^{k}+\overline{\mathbf{A}}_{n+\alpha}^{k}+\beta_{n+1}^{k} \mathbf{1}_{001}$.

Step 9. Add to row $m$ of the RFV, the vector $\mathbf{N}_{n+\alpha}^{T k} \mathbf{g}_{s}\left(\mathbf{u}_{s, n+1}^{k}, \mathbf{u}_{g s, n+1}^{k}\right)$. 
Step 10. Replace row $s$ of the RFV by $\mathbf{H}_{n+\alpha}^{T k} \mathbf{g}_{s}\left(\mathbf{u}_{s, n+1}^{k}, \mathbf{u}_{g s, n+1}^{k}\right)$.

where, at the row $s$ of the global vector of unknowns, the vector $\Delta \mathbf{u}_{s, n+1}^{k}$ has been conveniently replaced by the vector $\Delta \mathbf{u}_{s, n+1}^{\prime k}$ collecting the tangential components of the incremental relative slip displacement of the slave node $s$, defined as $\Delta \mathbf{u}_{s, n+1}^{\prime k}:=\left[\Delta u_{s 1, n+1}^{k}, \Delta u_{s 2, n+1}^{k}, 0\right]^{T}$, and, for the sake of compactness, the following matrices have been introduced (see Appendix 1),

$$
\begin{aligned}
& \mathbf{H}_{n+\alpha}^{k}:=\left[\boldsymbol{\tau}_{1, n+\alpha}^{k}, \tau_{2, n+\alpha}^{k}, \mathbf{0}\right] \\
& \mathbf{A}_{n+\alpha}^{k}:=\left[\alpha \mathbf{A}_{1, n+\alpha}^{T k} \mathbf{g}_{s}\left(\mathbf{u}_{s, n+1}^{k}, \mathbf{u}_{g s, n+1}^{k}\right), \alpha \mathbf{A}_{2, n+\alpha}^{T k} \mathbf{g}_{s}\left(\mathbf{u}_{s, n+1}^{k}, \mathbf{u}_{g s, n+1}^{k}\right), \mathbf{0}\right] \\
& \overline{\mathbf{A}}_{n+\alpha}^{k}:=\left[\alpha \overline{\mathbf{A}}_{1, n+\alpha}^{T k} \mathbf{g}_{s}\left(\mathbf{u}_{s, n+1}^{k}, \mathbf{u}_{g s, n+1}^{k}\right), \alpha \overline{\mathbf{A}}_{2, n+\alpha}^{T k} \mathbf{g}_{s}\left(\mathbf{u}_{s, n+1}^{k}, \mathbf{u}_{g s, n+1}^{k}\right), \mathbf{0}\right] \\
& \overline{\overline{\mathbf{A}}}_{n+\alpha}^{k}:=\left[\alpha \overline{\overline{\mathbf{A}}}_{1, n+\alpha}^{T k} \mathbf{g}_{s}\left(\mathbf{u}_{s, n+1}^{k}, \mathbf{u}_{g s, n+1}^{k}\right), \alpha \overline{\mathbf{A}}_{2, n+\alpha}^{T k} \mathbf{g}_{s}\left(\mathbf{u}_{s, n+1}^{k}, \mathbf{u}_{g s, n+1}^{k}\right), \mathbf{0}\right]
\end{aligned}
$$

where,

$$
\begin{aligned}
& \overline{\overline{\mathbf{A}}}_{1, n+\alpha}^{T k}:=\mathbf{N}_{\xi, n+\alpha}^{T k} m_{1, n+\alpha}^{\xi k}+\mathbf{N}_{\eta, n+\alpha}^{T k} m_{1, n+\alpha}^{\eta k} \\
& \overline{\overline{\mathbf{A}}}_{2, n+\alpha}^{T k}:=\mathbf{N}_{\xi, n+\alpha}^{T k} m_{2, n+\alpha}^{\xi k}+\mathbf{N}_{\eta, n+\alpha}^{T k} m_{2, n+\alpha}^{\eta k}
\end{aligned}
$$

and $\mathbf{1}_{001}$ is a $n_{\mathrm{dim}} \times n_{\mathrm{dim}}$ matrix with zero entries everywhere, except a 1 entry in the diagonal position $\left(n_{\mathrm{dim}}, n_{\mathrm{dim}}\right)$. The matrix $\beta_{n+1}^{k} \mathbf{1}_{001}$ is added in order to avoid the ill-conditioning (zero terms in the main diagonal) of the system, where the scalar parameter $\beta_{n+1}^{k}$ is defined as,

$$
\beta_{n+1}^{k}=\frac{1}{n_{\mathrm{dim}}} \mathbf{1}: \mathbf{K}_{s, s, n+1}^{k}=\frac{1}{n_{\mathrm{dim}}} \operatorname{tr}\left[\mathbf{K}_{s, s, n+1}^{k}\right]
$$

where $\operatorname{tr}[\cdot]$ denotes the trace operator and $n_{\text {dim }}$ is the number of dimensions of the problem.

Note that, following this procedure, the number of equations of the system is kept constant. Once convergence has been achieved, the increment of displacements $\Delta \mathbf{u}_{s, n+1}$ is computed in terms of $\Delta \mathbf{u}_{m, n+1}$ and $\Delta \mathbf{u}_{s, n+1}^{\prime}$ using either (53), for the quasi-static case, or (57) for the dynamic case.

The resulting global linearized system of equations for the frictionless contact case can be written in matrix form as,

$$
\left[\begin{array}{cccccc}
\mathbf{H}^{T} \mathbf{K}_{s, s} \mathbf{H}+\overline{\mathbf{A}}+\beta \mathbf{1}_{001} & \mathbf{H}^{T} \mathbf{K}_{s, g s} & \mathbf{0} & \mathbf{H}^{T} \mathbf{K}_{s, s} \mathbf{N}+\mathbf{A}^{T} & \mathbf{0} & \mathbf{0} \\
\mathbf{K}_{g s, s} \mathbf{H} & \mathbf{K}_{g s, g s} & \mathbf{K}_{g s, g 1} & \mathbf{K}_{g s, s} \mathbf{N} & \mathbf{0} & \mathbf{0} \\
\mathbf{0} & \mathbf{K}_{g 1, g s} & \mathbf{K}_{g 1, g 1} & \mathbf{0} & \mathbf{0} & \mathbf{0} \\
\mathbf{N}^{T} \mathbf{K}_{s, s} \mathbf{H}+\overline{\overline{\mathbf{A}}} & \mathbf{N}^{T} \mathbf{K}_{s, g s} & \mathbf{0} & \mathbf{K}_{m, m}+\mathbf{N}^{T} \mathbf{K}_{s, s} \mathbf{N} & \mathbf{K}_{m, g m} & \mathbf{0} \\
\mathbf{0} & \mathbf{0} & \mathbf{0} & \mathbf{K}_{g m, m} & \mathbf{K}_{g m, g m} & \mathbf{K}_{g m, g 2} \\
\mathbf{0} & \mathbf{0} & \mathbf{0} & \mathbf{0} & \mathbf{K}_{g 2, g m} & \mathbf{K}_{g 2, g 2}
\end{array}\right]_{n+1}^{k}\left[\begin{array}{c}
\Delta \mathbf{u}_{s}^{\prime} \\
\Delta \mathbf{u}_{g s} \\
\Delta \mathbf{u}_{g 1} \\
\Delta \mathbf{u}_{m} \\
\Delta \mathbf{u}_{g m} \\
\Delta \mathbf{u}_{g 2}
\end{array}\right]_{n+1}^{k}=-\left[\begin{array}{c}
\mathbf{H}^{T} \mathbf{g}_{s} \\
\mathbf{g}_{g s} \\
\mathbf{g}_{g 1} \\
\mathbf{g}_{m}+\mathbf{N}^{T} \mathbf{g}_{s} \\
\mathbf{g}_{g m} \\
\mathbf{g}_{g 2}
\end{array}\right]_{n+1}^{k}
$$

where note that the matrices $\mathbf{N}, \mathbf{H}, \mathbf{A}, \overline{\mathbf{A}}, \overline{\overline{\mathbf{A}}}$ are evaluated at the configuration $n+\alpha$, as indicated in (66)-(68). 
Remark 3. Note that, contrary to what happens for the full stick friction case, block-symmetry of the resulting global tangent stiffness matrix for the frictionless case is lost due to the contributions of the matrices $\mathbf{A}_{n+\alpha}^{k}, \overline{\mathbf{A}}_{n+\alpha}^{k}, \overline{\overline{\mathbf{A}}}_{n+\alpha}^{k}$ given in (67). Matrices $\mathbf{A}_{n+\alpha}^{k}$ and $\overline{\mathbf{A}}_{n+\alpha}^{k}$ arise from the variation of the orthogonal unit tangent vectors $\tau_{1, n+\alpha}^{k}$ and $\tau_{2, n+\alpha}^{k}$ and note that $\mathbf{A}_{n+\alpha}^{k} \neq \overline{\mathbf{A}}_{n+\alpha}^{T k}$, while matrix $\overline{\overline{\mathbf{A}}}_{n+\alpha}^{k}$ arises from the variation of $\mathbf{N}_{n+\alpha}^{k}$ in (65) $)_{1}$. Note also that, for linear elements the matrix $\overline{\mathbf{A}}_{n+\alpha}^{k}=\mathbf{0}$ (see Appendix 1). Within an infinitesimal strain framework, those variations can be neglected and the block-symmetry of the resulting global tangent stiffness matrix can be preserved.

\subsubsection{Update of slave and master displacements and contact status}

The displacements of the master nodes are updated according to the following expression,

$$
\mathbf{u}_{m, n+1}^{k+1}=\mathbf{u}_{m, n+1}^{k}+\Delta \mathbf{u}_{m, n+1}^{k}
$$

The displacements of the slave nodes are updated according to the following procedure:

Step 1. Compute the current displacements and coordinates of the slave node at the iteration $k+1$ of time $n+\alpha$, using the isoparametric coordinates $\overline{\boldsymbol{\xi}}_{n+\alpha}^{k}$ of the closest-pointprojection at the iteration $k$,

$$
\begin{aligned}
& \overline{\mathbf{u}}_{s, n+1}^{k+1}=\mathbf{u}_{s, n+1}^{k}+\mathbf{N}_{n+\alpha}^{k} \Delta \mathbf{u}_{m, n+1}^{k}+\Delta u_{s 1, n+1}^{k} \tau_{1, n+\alpha}^{k}+\Delta u_{s 2, n+1}^{k} \tau_{2, n+\alpha}^{k} \\
& \overline{\mathbf{x}}_{s, n+1}^{k+1}=\mathbf{X}_{s}+\overline{\mathbf{u}}_{s, n+1}^{k+1}
\end{aligned}
$$

Step 2. Update the isoparametric coordinates $\overline{\boldsymbol{\xi}}_{n+\alpha}^{k+1}$ of the closest-point-projection of the slave node at the iteration $k+1$ as follows:

For the quasi-static case, the configuration $n+\alpha$ is set equal to $n+1$, and $\overline{\boldsymbol{\xi}}_{n+1}^{k+1}$ is computed using the coordinates $\overline{\mathbf{x}}_{s, n+1}^{k+1}$ of the slave node.

For the dynamic case, the configuration $n+\alpha$ is chosen as the mid-point configuration, setting $n+\alpha$ equal to $n+1 / 2$, and $\bar{\xi}_{n+1 / 2}^{k+1}$ is computed using the coordinates $\overline{\mathbf{x}}_{s, n+1 / 2}^{k+1}$ of the slave node defined as,

$$
\overline{\mathbf{x}}_{s, n+1 / 2}^{k+1}=\frac{1}{2}\left(\overline{\mathbf{x}}_{s, n+1}^{k+1}+\mathbf{x}_{s, n}\right)
$$

Step 3. Update the current displacements of the slave node according to the following procedure:

For the quasi-static case, the current displacements of the slave node at the iteration $k+1$ of time $n+1$ are updated as,

$$
\mathbf{u}_{s, n+1}^{k+1}=\mathbf{N}_{n+1}^{k+1} \mathbf{x}_{m, n+1}^{k+1}-\mathbf{X}_{s}
$$

For the dynamic case, the current displacements of the slave node at the iteration $k+1$ of time $n+1$ are updated as, 


$$
\mathbf{u}_{s, n+1}^{k+1}=\overline{\mathbf{x}}_{s, n+1}^{k+1}-\Delta g_{N, n+1 / 2}^{k+1} \mathbf{n}_{n+1 / 2}^{k+1}-\mathbf{X}_{s}
$$

where,

$$
\Delta g_{N, n+1 / 2}^{k+1}=\left(\overline{\mathbf{x}}_{s, n+1}^{k+1}-\mathbf{x}_{s, n}-\mathbf{N}_{n+1 / 2}^{k+1}\left(\mathbf{x}_{m, n+1}^{k+1}-\mathbf{x}_{m, n}\right)\right) \cdot \mathbf{n}_{n+1 / 2}^{k+1}
$$

such that, it is ensured that the discrete frictionless contact kinematic constraint given by (56) is satisfied, and the discrete linear momentum, angular momentum and energy are conserved.

For the dynamic case, once the displacements of the slave and master nodes have been updated, the nodal velocities of the slave and master nodes are updated using (19).

Once the displacements have been updated, the contact status has to be checked out in order to decide if it has to be keep as active or if it has to be deactivated for the next step. The contact will be still active if the contact normal force $f_{N_{n+\alpha}}:=\mathbf{f}_{s, n+\alpha} \cdot \mathbf{n}_{n+\alpha}$ satisfies the following condition:

$$
f_{N_{n+\alpha}}:=\mathbf{f}_{s, n+\alpha} \cdot \mathbf{n}_{n+\alpha}=\mathbf{g}_{s}\left(\mathbf{u}_{s, n+1}, \mathbf{u}_{g s, n+1}\right) \cdot \mathbf{n}_{n+\alpha} \geq 0
$$

Otherwise, contact is lost and the contact status for the slave node $s$ has to be deactivated for the next time step.

\subsection{Finite element implementation of the direct elimination algorithm for contact problems}

The direct method proposed in this work is relatively easy to implement into a FE code. A detailed description of the matrix operations needed to be carrried out for the full stick frictional case and the frictionless case has been given above in a step-by-step procedure. The finite element formulation and direct elimination algorithm for quasi-static and dynamic analysis of full stick friction and frictionless contact problems have been implemented in an enhanced version of the finite element code for structural analysis RamSeries [48]. Figure 2 shows a flow chart of the direct elimination algorithm for contact problems. 


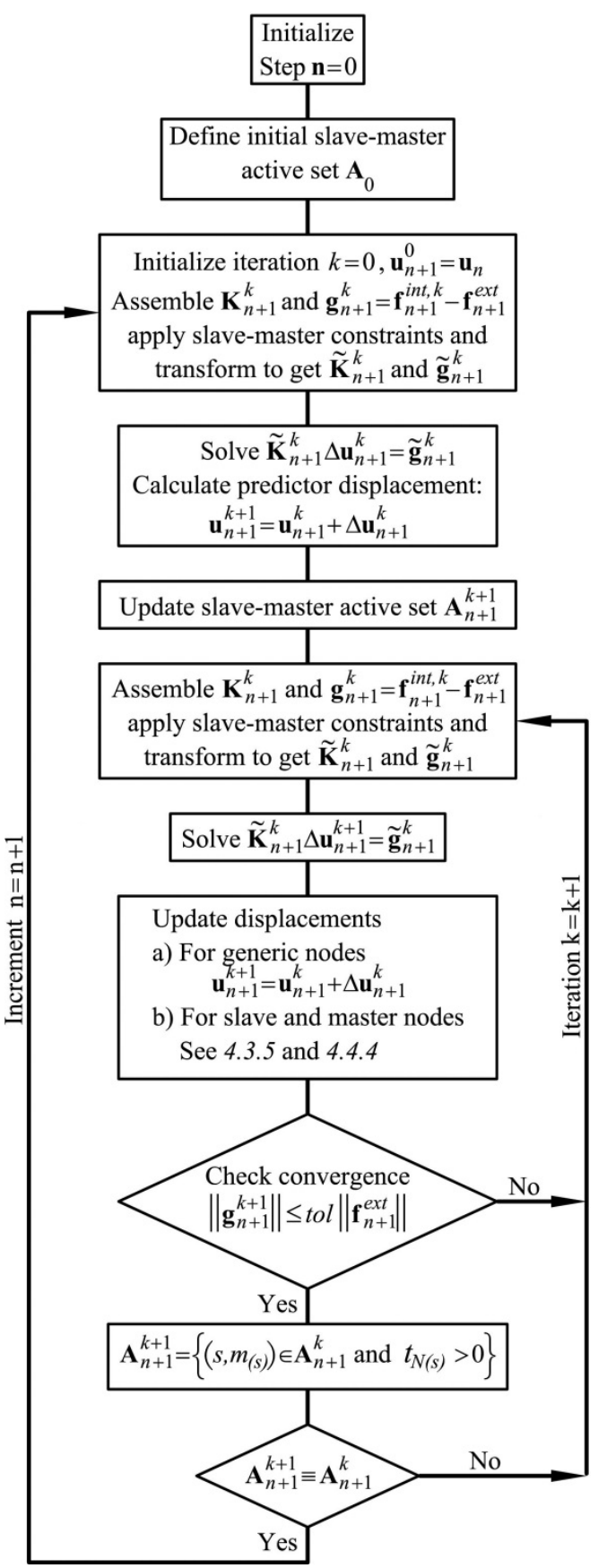

Figure 2. Implementation chart of the proposed contact formulation into a finite element code.

\section{Numerical examples}

In this section a selection of representative quasi-static and dynamic numerical examples, that illustrate the performance of the contact formulation proposed, is shown. Three quasi-static and one dynamic numerical examples have been chosen.

First, a contact patch test is considered. An assessment of the error obtained using the direct elimination method, for different mesh sizes and different Young's modulus, has been performed. In the second example, a Hertzian contact problem [23] is considered in order to make an assessment of the accuracy of the proposed contact model. Numerical results obtained using the direct elimination method, have been compared with analytical solutions [23]. The 
third example consists of a 3D axisymmetric punch indentation benchmark test. This is one of the benchmark tests presented by Konter (2005) [28] within the FENET EU Thematic Network Report called Advanced Finite Element Contact Benchmarks. Numerical results obtained for the 3D punch indentation benchmark test using the proposed contact formulation, have been compared with numerical results obtained with the commercial FE softwares Abaqus/Standard and MSC.MARC, using the penalty method [28]. The fourth example deals with the dynamic impact of two rigid cylinders. Here the goal is to show that the proposed formulation exhibits the conservation of the discrete linear momentum, angular momentum and total energy.

The numerical simulations have been performed using an enhanced version of the finite element program RamSeries [48] developed by COMPASS, a spin-off company of CIMNE.

\subsection{Contact patch test}

It is well known that node-to-segment (NTS) formulations, such as the one shown in this work, do not satisfy the patch test. This example deals with the contact of two elastic blocks. An assessment of the error obtained using the direct elimination method, for different mesh sizes and different Young's modulus, has been performed. Full stick frictional conditions have been considered. The mesh refinement is controlled by the number of divisions per unit of length of the mesh of the master surface, and the following two values of the ratio between the mesh sizes of the master and slave surfaces: 0.75 and 1.5. Two sets of values for the elastic Young's modulus have been considered for both the slave and master bodies: 2.1E+11 Pa and 5.0E+07 Pa. Standard Galerkin P1 linear displacement hexahedral elements have been used. Figure 3 shows the geometry, material properties and boundary conditions for the contact patch test.

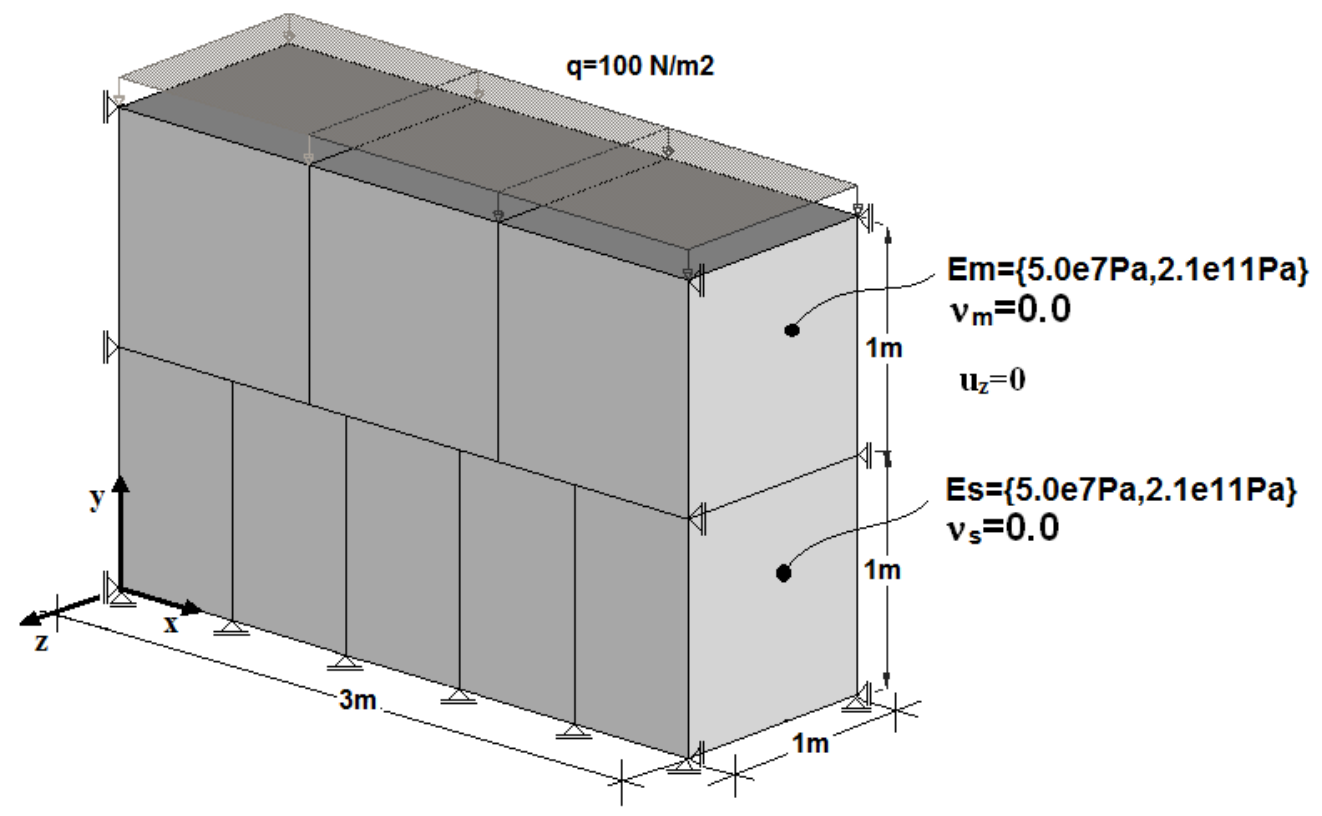

Figure 3. Geometry, material properties and boundary condition for contact patch test.

The estimated error has been computed as: 


$$
\operatorname{Error}(\%)=100 \frac{\sigma_{\text {true }}-\sigma_{\text {max } \min }}{\sigma_{\text {true }}}
$$

where $\sigma_{\text {true }}=100 \mathrm{~Pa}$ would be the exact value of the stress at the contact interface if the patch test would be verified, and $\sigma_{\text {max,min }}$ is the value of the maximum or minimum stress (the one which is less close to the exact stress value) computed by the proposed method.

Figure 4 shows the convergence to the exact solution of the contact pressure when the mesh is refined, for two values of the ratio between the master mesh size and the slave mesh size (0.75 and 1.50), and for four different sets of the Young's elastic modulus $(2.11 \mathrm{E}+11 \mathrm{~Pa}$ and 5.0E+07 Pa) for the slave and master bodies. Results show that the convergence increases when the ratio between the master and slave mesh sizes increases. It is also shown that the convergence increases when the mechanical properties (Young's elastic modulus) of the two bodies are similar.

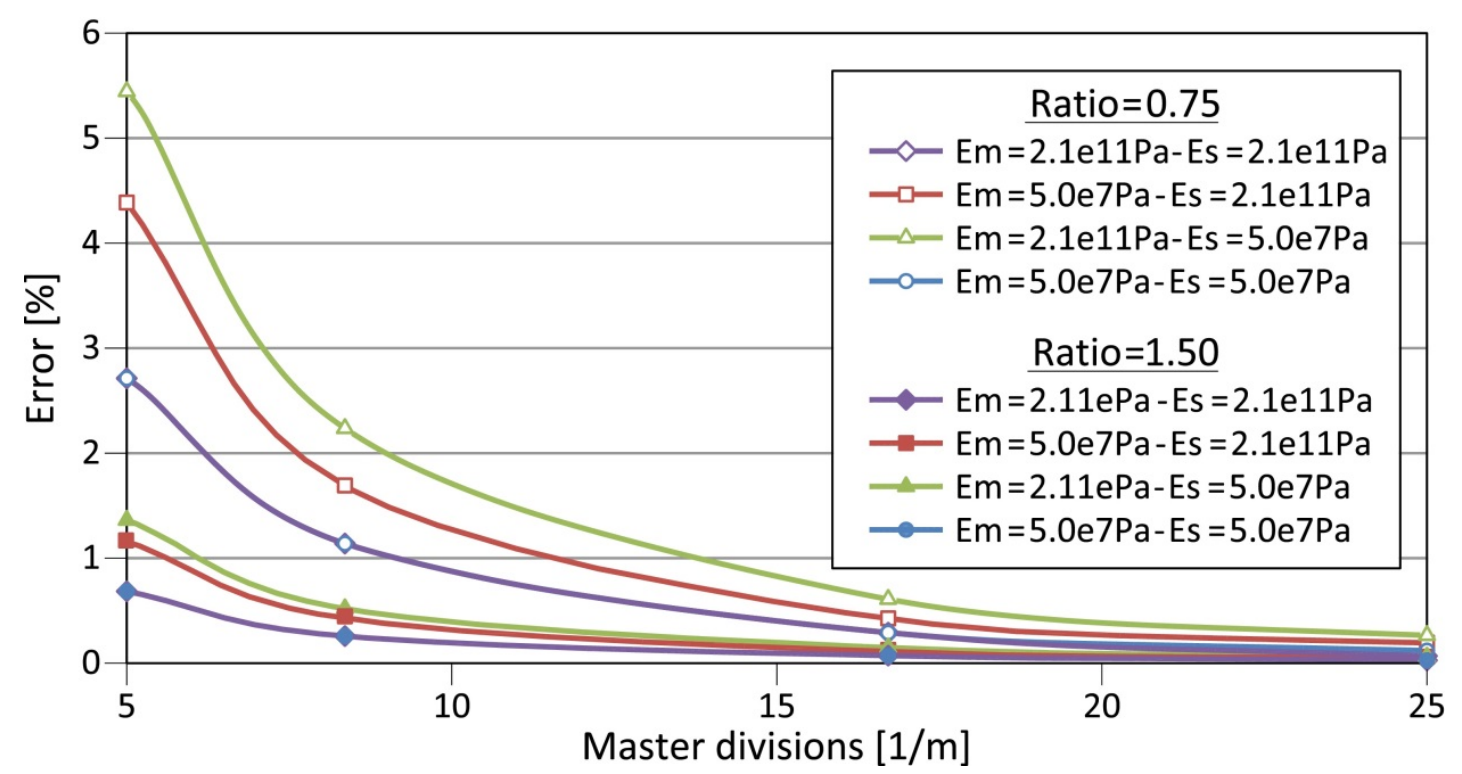

Figure 4. Contact pressure error. Convergence behavior for different number of master surface divisions, for four different sets of the elastic Young's modulus and for two different master/slave mesh size ratios.

\subsection{Hertzian contact test}

In order to make an assessment of the accuracy of the proposed contact formulation, the Hertzian contact test between two elastic cylinders has been considered [23]. Numerical results obtained using the direct elimination method have been compared with analytical solutions [23]. Frictionless conditions have been assumed. Standard Galerkin P1 linear displacement hexahedral elements have been used. Symmetry conditions have been introduced and only a quarter of each one of the cylinders has been considered. The finite element mesh used for the discretization of the two cylinders consists of 3137 elements and 6438 nodes. The geometry, mesh and boundary conditions are shown in Figure 5. 


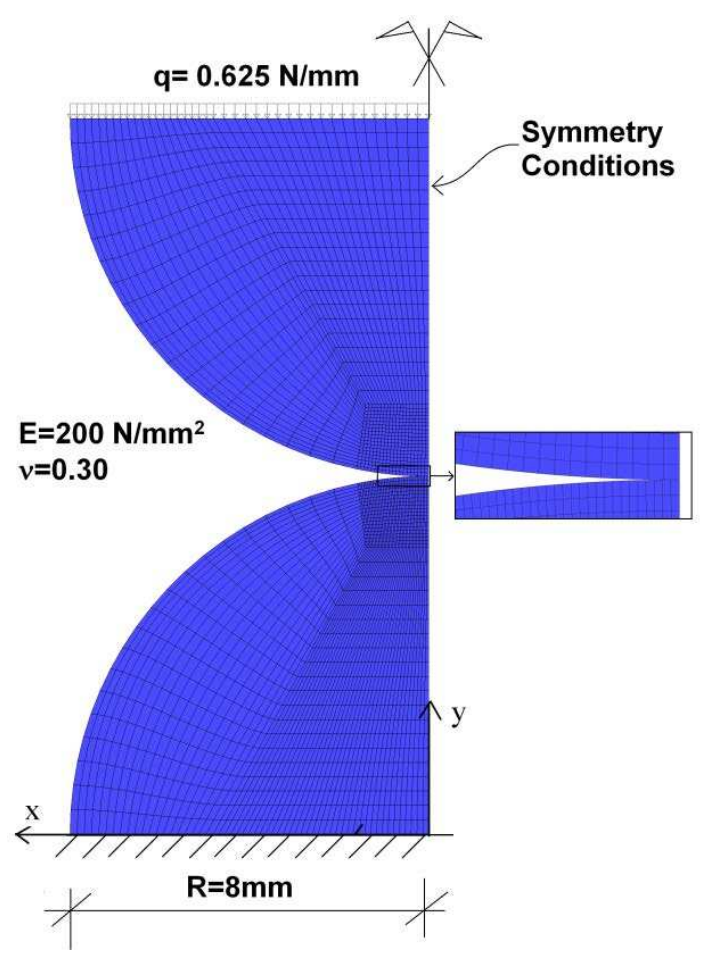

Figure 5. Hertzian contact test problem.

Using the contact formulation proposed in this work, the computed width of the contact area, given by the position of the nodes of the last master element contacted, can be estimated that is within the following range of values:

$$
0.67 \mathrm{~mm} \leq b_{\text {num }} \leq 0.71 \mathrm{~mm}
$$

The exact width of the contact area can be computed analytically using the following expression:

$$
b=2 \sqrt{2 q R^{2} \frac{1-v^{2}}{\pi E}}=0.681 \mathrm{~mm}
$$

where $R$ is the radius of the cylinder and $q$ is the vertical pressure on the top. Therefore, the exact value of the contact width falls within the range of values computed numerically.

The exact distribution of the contact pressure can be computed analytically using the following expression,

$$
t_{N}=t_{N}^{\max } \sqrt{1-\frac{x}{b}}, \quad t_{N}^{\max }=\sqrt{\frac{2 q E}{\pi\left(1-v^{2}\right)}}
$$

where $t_{N}^{\max }$ is the maximum contact normal pressure.

Figure 6 shows the comparison between the numerical and the analytical results for the contact normal pressure. A very good agreement can be observed. 


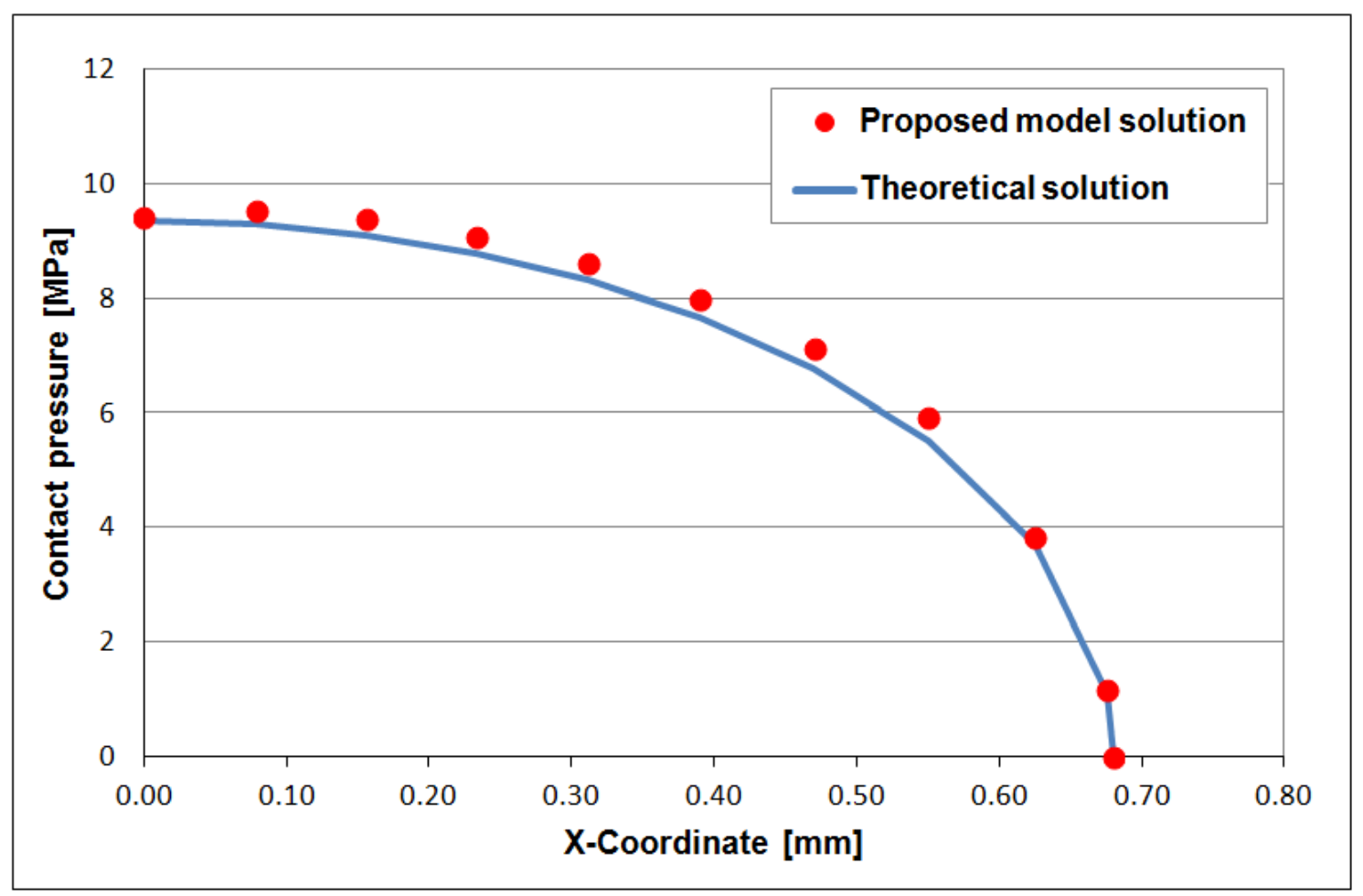

Figure 6. Contact normal pressure distribution.

\subsection{D Punch indentation benchmark test}

This example deals with a 3D punch indentation benchmark test [27]. An axisymmetric steel punch is applied on an aluminum cylinder. The height of the steel punch is $100 \mathrm{~mm}$ and its radius is $50 \mathrm{~mm}$. The bottom corner of the punch is rounded with a radius of $10 \mathrm{~mm}$. The radius of the aluminum cylinder is $100 \mathrm{~mm}$ and its height is $200 \mathrm{~mm}$. The elastic Young's modulus of the steel punch and the aluminum cylinder are $2.1 \mathrm{E}+11 \mathrm{~Pa}$ and $0.7 \mathrm{E}+11 \mathrm{~Pa}$, respectively. The Poisson's coefficient of both the steel punch and the aluminum cylinder is 0.3. An axial uniform normal pressure of $100 \mathrm{MPa}$ is applied on the top surface of the steel punch. Zero prescribed displacements are considered on the bottom surface of the aluminum cylinder. Frictionless conditions have been considered. Taking into account the axisymmetric nature of the problem, only a quarter of the geometry of the bodies has been considered, adding the corresponding symmetry conditions. Standard Galerkin P1 linear displacement hexahedral elements have been used. The finite element mesh used for the discretization of the punch and the cylinder consists of 7201 elements and 8386 nodes. Figure 7 shows the finite element discretization of the 3D punch indentation benchmark test. 


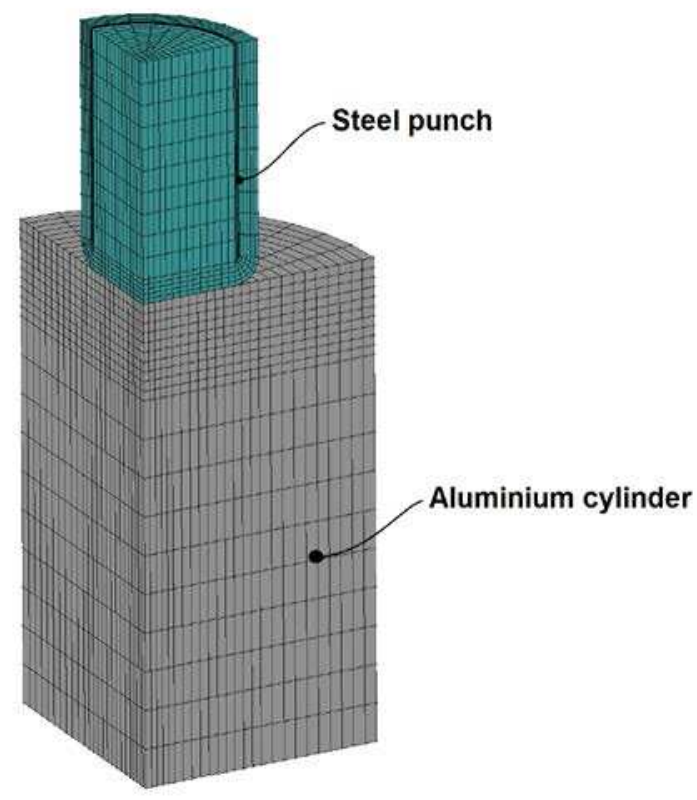

Figure 7. Finite element meshes used for the 3D punch indentation benchmark test.

The numerical results obtained for the 3D punch indentation benchmark test using the proposed direct elimination contact formulation have been compared with numerical results obtained with the commercial FE softwares Abaqus/Standard and MSC.MARC, using the penalty method and standard Galerkin P1 linear displacement hexahedral elements [28].

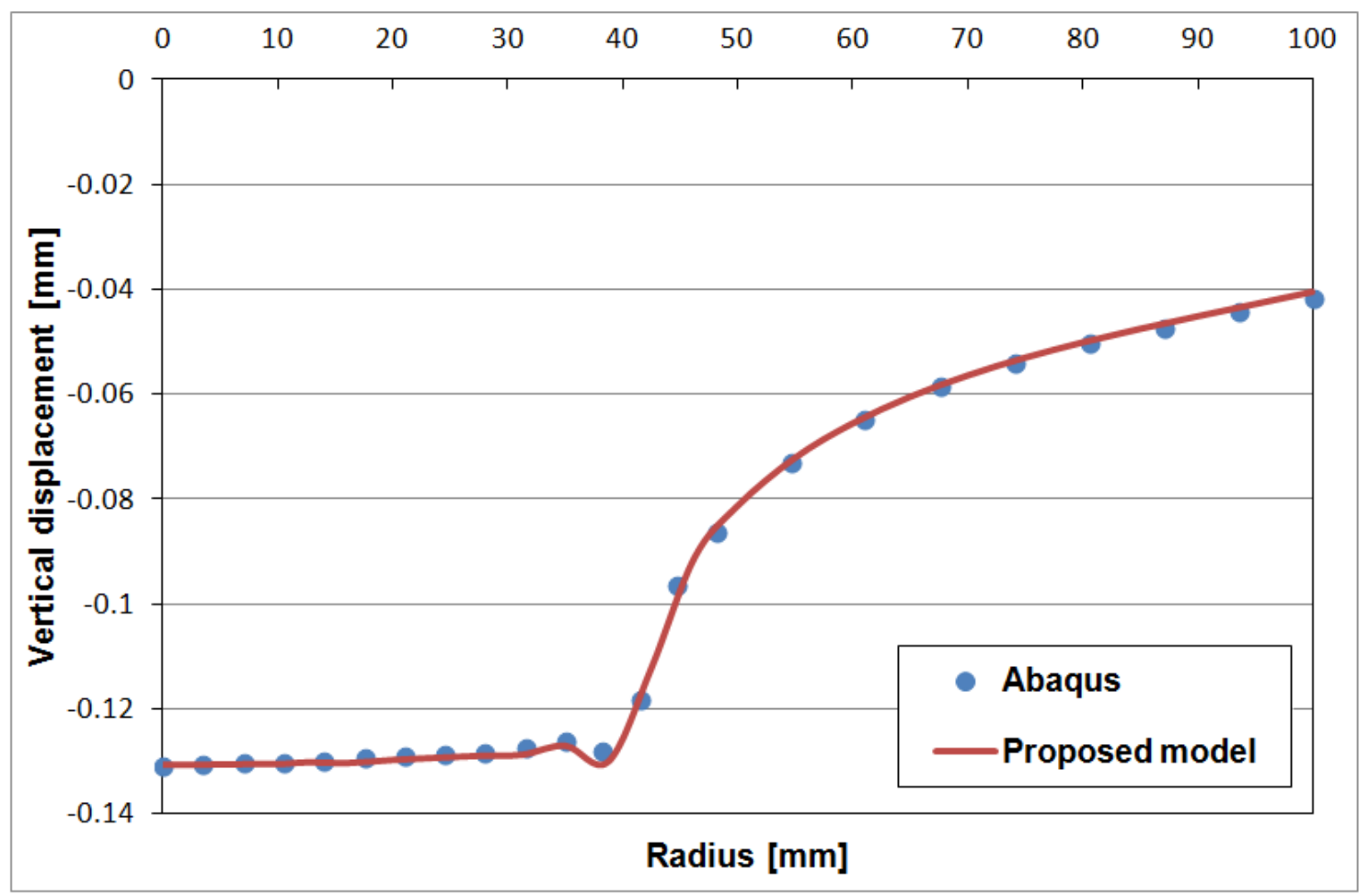

Figure 8. Vertical displacement of the top surface of the aluminium cylinder as a function of the radial distance. 


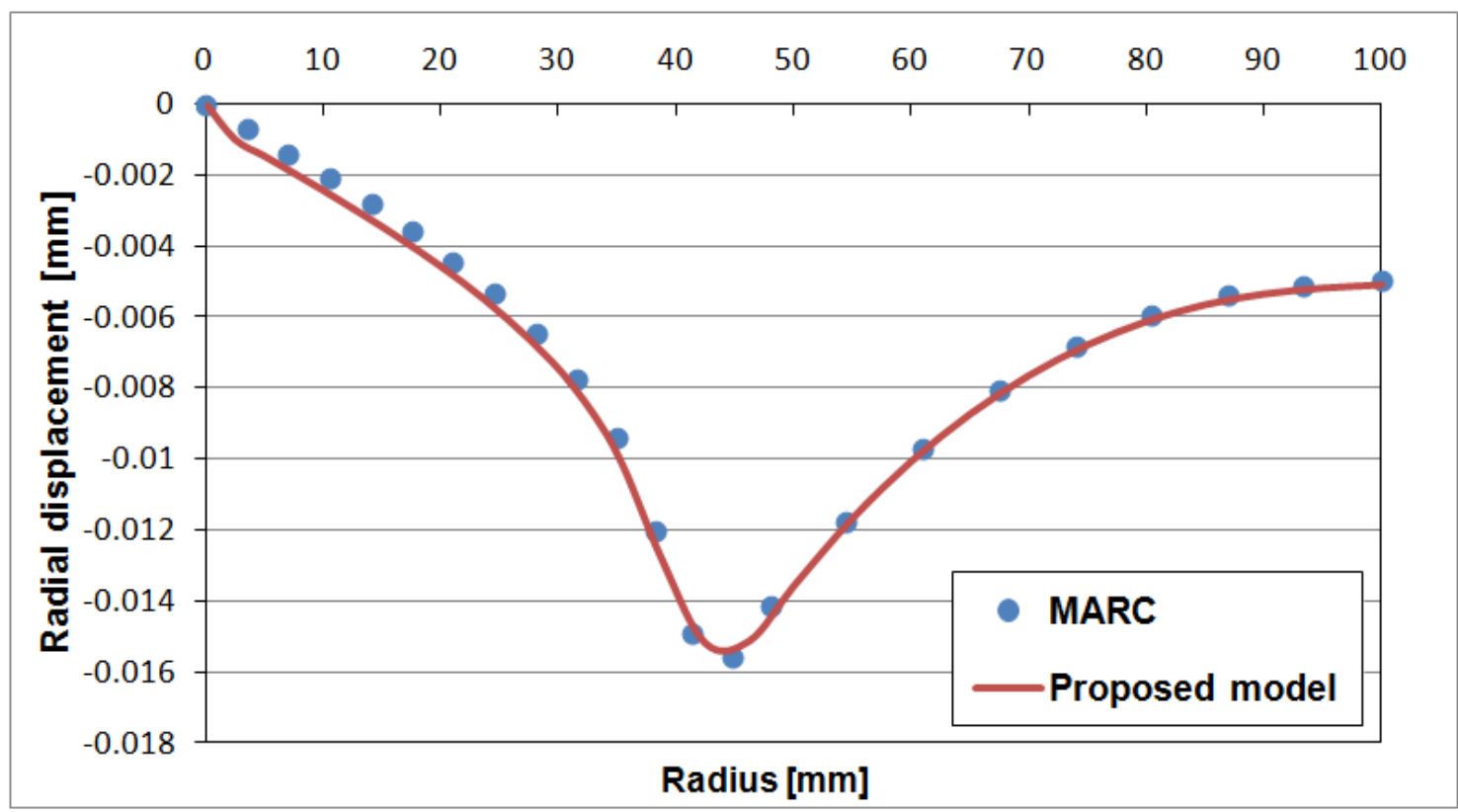

Figure 9. Radial displacement of the top surface of the aluminium cylinder as a function of the radial distance.

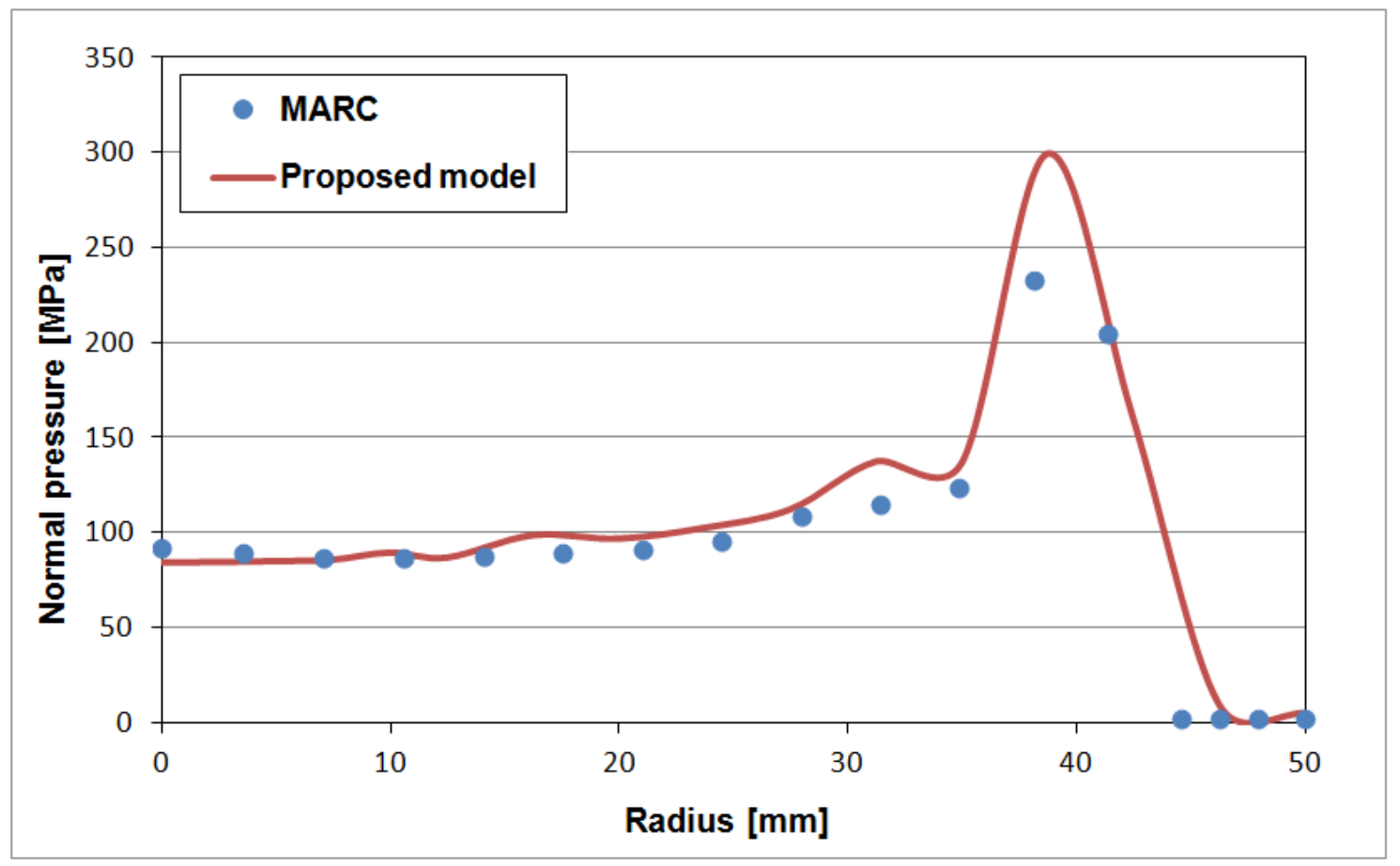

Figure 10. Contact pressure on the top surface of the aluminium cylinder as a function of the radial distance.

Figure 8 shows the distribution of the vertical displacement of the top surface of the aluminium cylinder as a function of the radial distance. Numerical results obtained in this work are compared with results obtained using the penalty method with Abaqus/Standard [28]. A very good agreement is shown.

Figure 9 shows the distribution of the radial displacement of the top surface of the aluminium cylinder as a function of the radial distance. Numerical results obtained in this work 
are compared with results obtained using the penalty method with MSC.MARC [28]. Once again, a very good agreement is shown.

Figure 10 shows the distribution of the contact normal pressure vs the radial distance at the top surface of the aluminium cylinder. Numerical results obtained in this work are compared with results obtained using the penalty method with MSC.MARC [28]. A very good agreement is shown except in the peak area, for a radius around $40 \mathrm{~mm}$, where some differences can be observed.

\subsection{Impact of two rigid cylinders}

This example leads with the impact of two quasi-rigid cylinders sliding on a rigid surface. Frictionless contact conditions between the two cylinders and between each one of the cyinders and the sliding surface have been considered. The radius of the cylinders is $0.1 \mathrm{~m}$. Elastic SaintVenant material models with Young's elastic modulus 2.1E+09 Pa and Poisson's coefficient 0.3 have been considered. The mass density of the material is $2500 \mathrm{Kg} / \mathrm{m}^{3}$. Cylinder 1 has an initial velocity in the $\mathrm{x}$-direction of $1.0 \mathrm{~m} / \mathrm{sec}$.

The cylinders have been discretized using standard Galerkin P1 linear displacement tetrahedral finite elements. The finite element mesh used for the discretization of the two cylinders consists of 4672 elements and 1224 nodes. Figure 11 shows the geometry, initial conditions and finite element meshes of the two cylinders. Time increments of $0.001 \mathrm{sec}$ have been considered.

Figure 12 shows the evolution of the positions of the two cylinders at different time steps. Despite the fact that frictionless conditions have been considered, Figure 12 shows that the cylinders rotate around their axes. This is an undesirable effect due to the finite element discretization of the cylinders. Unless an extremely fine mesh is used, the unit normal to the master surface at the closest-point-projection does not pass through the axis of the cylinder, leading to a moment when the cylinders come into contact. A similar effect can be found in the results shown in [5]. Despite those undesirable effects, the proposed contact model is able to conserve the discrete total linear momentum and angular momentum, and the total energy, as it is shown on Figures 13 and 14, respectively.

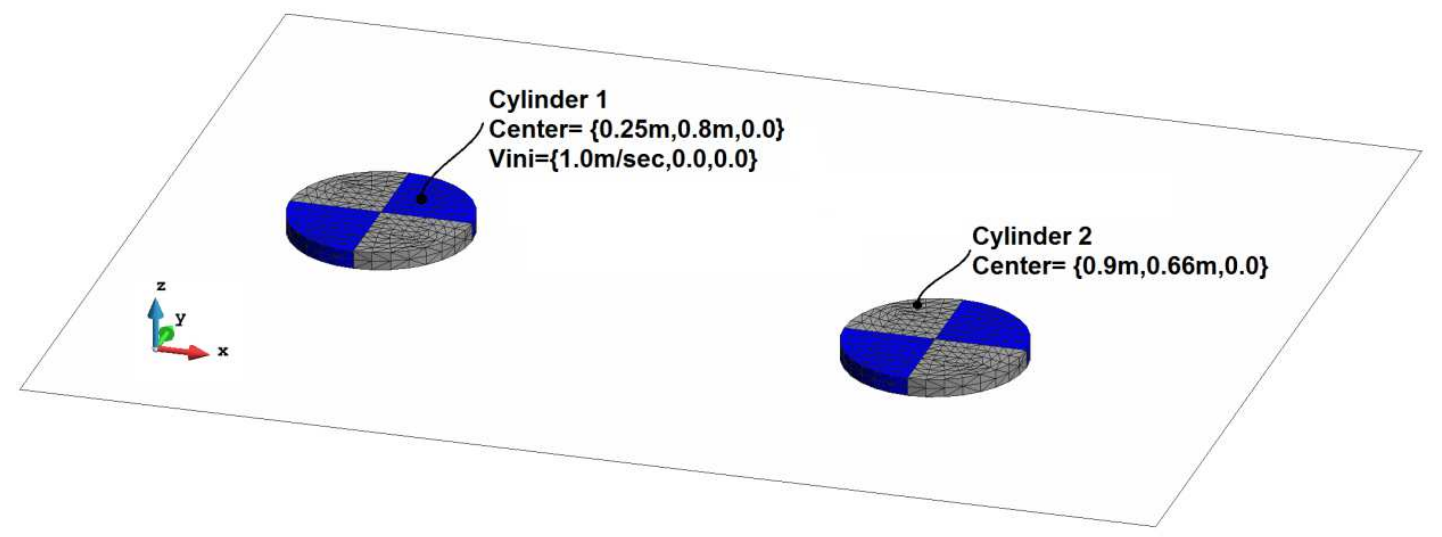

Figure 11. Impact of two quasi-rigid cylinders. Geometry, mesh and initial conditions. 

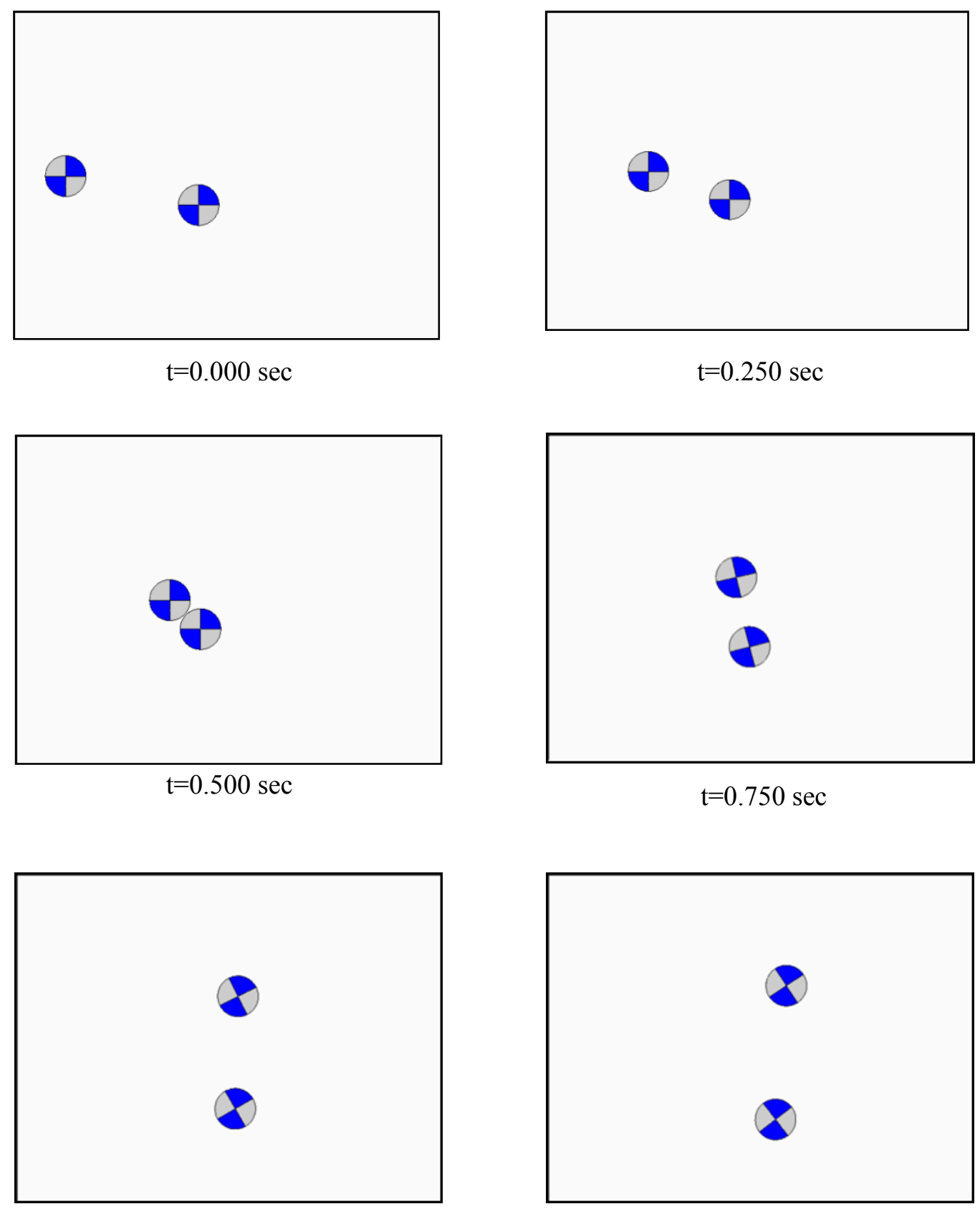

$\mathrm{t}=1.000 \mathrm{sec}$

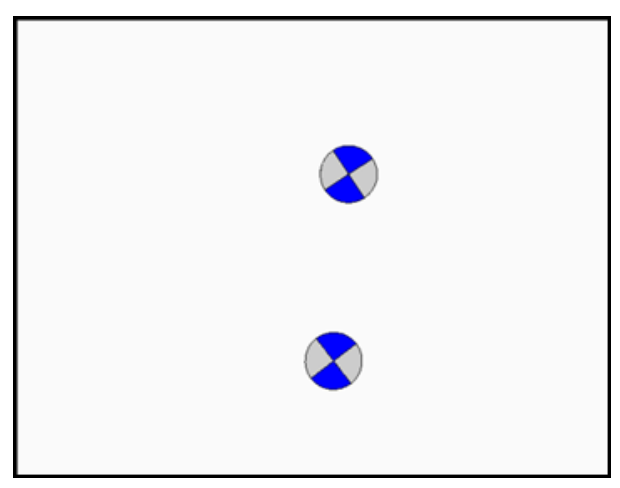

$\mathrm{t}=1.125 \mathrm{sec}$

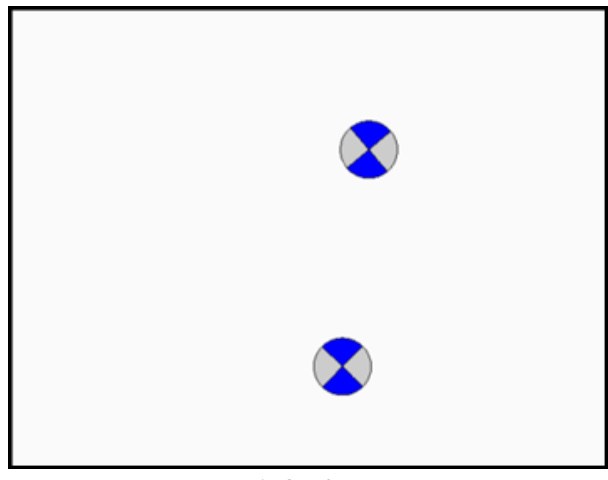

$\mathrm{t}=1.250 \mathrm{sec}$

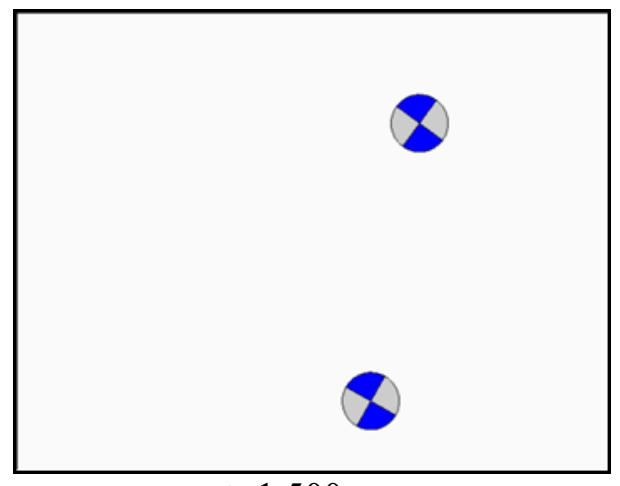

$\mathrm{t}=1.500 \mathrm{sec}$

Figure 12. Impact of two quasi-rigid cylinders. Configurations at different time steps. 


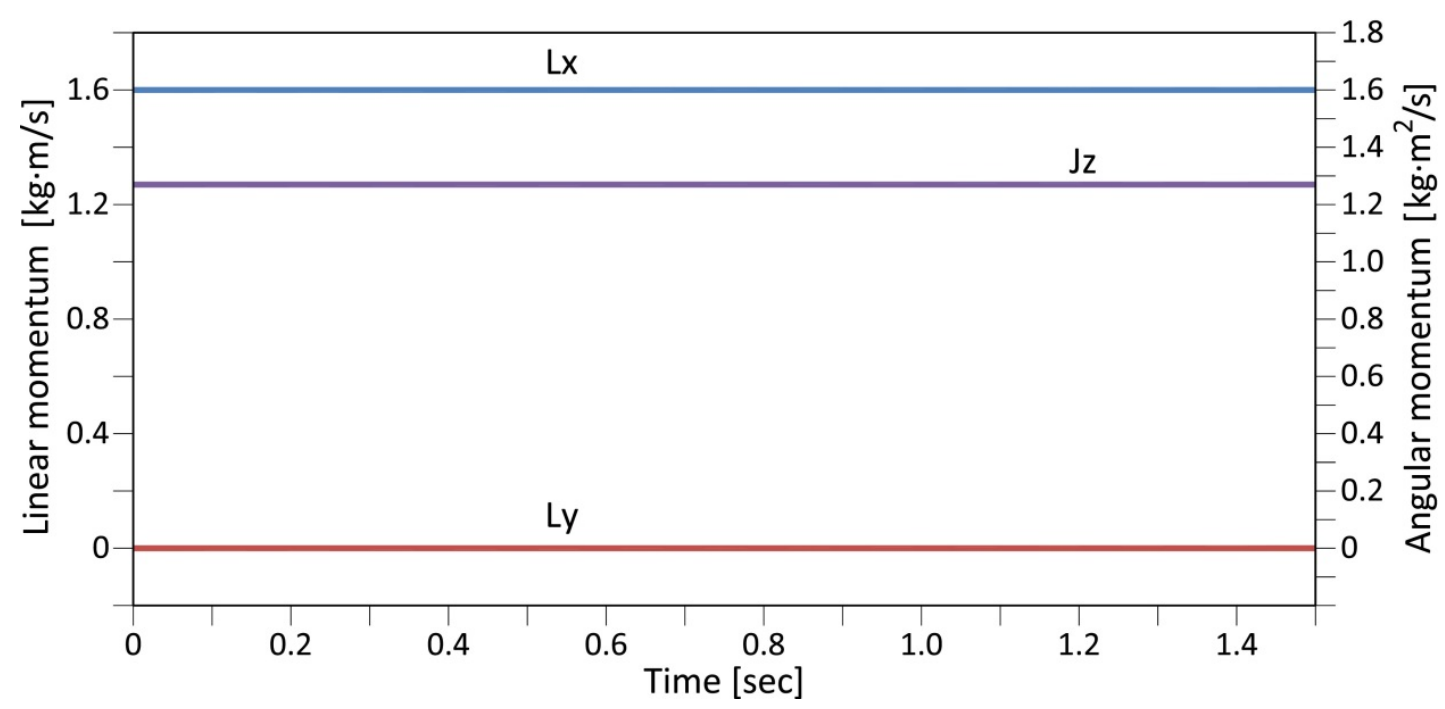

Figure 13. Impact of two quasi-rigid cylinders. Evolution of the two components of the linear momentum (Lx and Ly) and the angular momentum (Jz).

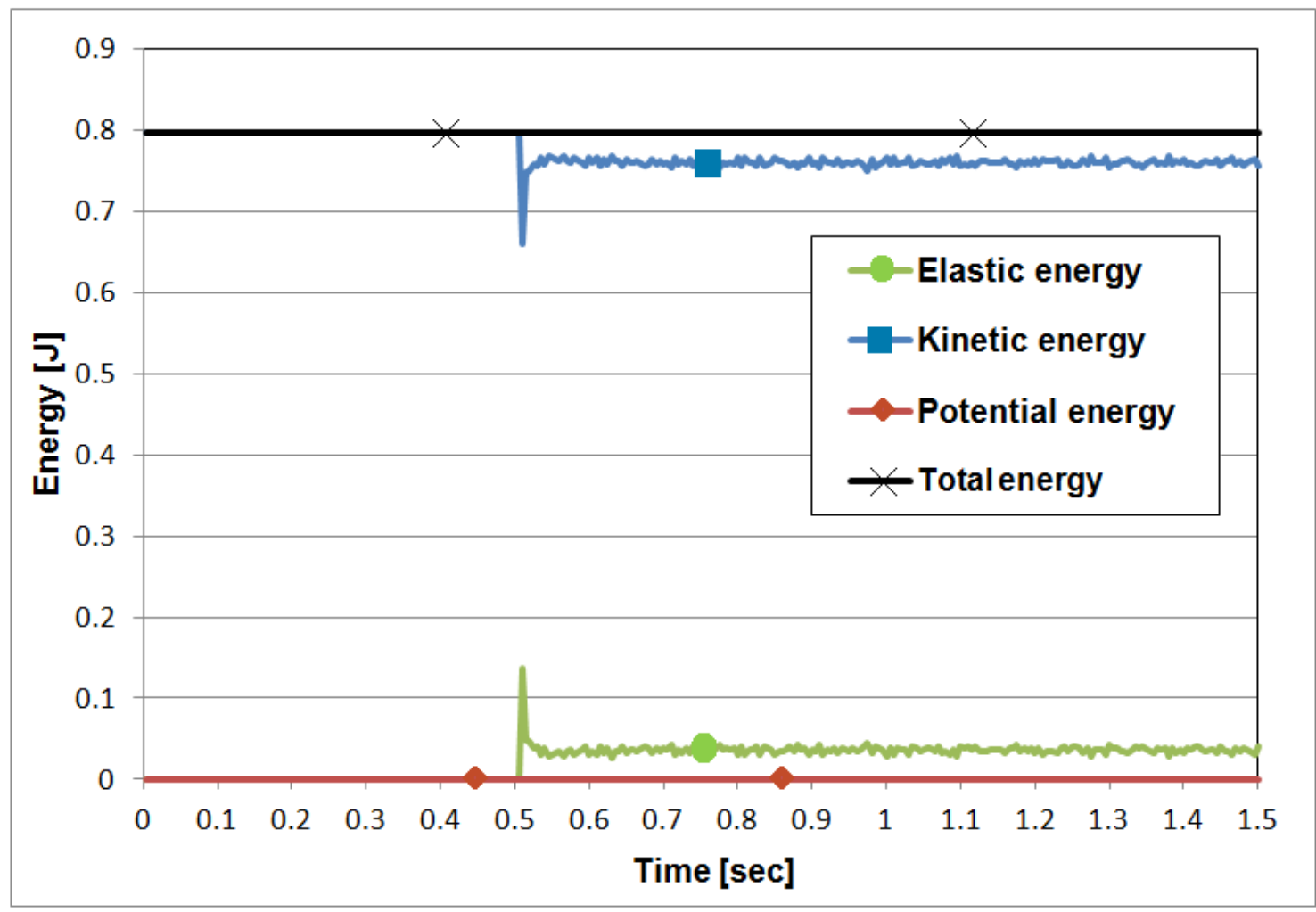

Figure 14. Impact of two quasi-rigid cylinders. Evolution of the different energies.

\section{Conclusions}

In this paper a new formulation for quasi-static and dynamic contact problems, under full stick friction and frictionless contact conditions, has been developed and implemented. The constraints arising in full stick and frictionless contact problems are imposed in a strong fashion by a direct elimination of the involved degrees of freedom of the resulting system of equations. Drawbacks inherent to the penalty method, such as the selection of suitable penalty parameters 
or the ill-conditioning of the system, are fully avoided. Furthermore, drawbacks linked to the Lagrange multipliers method, such as the need to dynamically expand the system of equations, adding new equations for the Lagrange multipliers, are also bypassed. Remarkably, the contact constraints are easily imposed by a number of transformations applied to the tangent stiffness matrix and residual vector of the problem without contact constraints. For contact dynamic problems, a conserving implicit time stepping algorithm has been presented and it has been shown that for the frictionless case it preserves the conservation of angular momentum and energy, as exhibited by the continuum, while for the full stick friction case, only energy is conserved.

The performance of the contact formulation has been shown in a number of representative examples. It is well known that node-to-segment (NTS) based contact formulations, as the one shown here, do not pass the contact patch test. Nevertheless, the results obtained in the contact patch test show a convergence to the exact solution as the mesh is refined and the ratio between the master size elements and slave size elements increases. An assessment of the formulation has been performed in the analysis of two quasi-static benchmark tests. A very good correlation between numerical results obtained in this work and analytical (Hertz contact test) or numerical results, obtained with Abaqus and Marc FE codes, using the penalty method (3D punch indentation benchmark test), has been obtained. Finally, an assessment of the conserving implicit time-stepping algorithm is shown in the numerical simulation of a dynamic contact problem. The impact of two rigid bodies is considered and it is shown that discrete linear momentum, angular momentum and total energy are conserved.

As a final concluding remark, we would like to point out that despite the fact that over the last decade an important effort has been done looking for contact formulations which had as a design target the requirement to pass the patch test, those formulations usually imply an important additional computational cost, while node-to-segment (NTS) contact formulations can still provide very good results, being competitive for many engineering applications.

\section{Acknowledgements}

Authors would like to acknowledge COMPASS for providing the code RamSeries where the contact algorithms shown in this work have been implemented. Collaborations of Dr. Julio Garcia and Eng. Jaume Sagues, as well as the support of the students Massimo Angelini and Matias Bossio, are gratefully acknowledged.

\section{Appendix 1}

This Appendix contents the variation of the orthogonal unit tangent vectors $\tau_{1, n+\alpha}$ and $\tau_{2, n+\alpha}$ at the configuration $n+\alpha$ of the closest-point-projection on the master surface.

Covariant tangent vectors $\tau_{\xi, n+\alpha}$ and $\tau_{\eta, n+\alpha}$ to the isoparametric coordinates at the closest-point-projection at the configuration $n+\alpha$ are defined as,

$$
\boldsymbol{\tau}_{\xi, n+\alpha}=\mathbf{N}_{\xi, n+\alpha} \mathbf{x}_{m, n+\alpha}, \quad \tau_{\eta, n+\alpha}=\mathbf{N}_{\eta, n+\alpha} \mathbf{x}_{m, n+\alpha}
$$

where the $n_{\text {dim }} \times n_{\text {dim }} n_{m n o d}$ matrices $\mathbf{N}_{\xi, n+\alpha}, \mathbf{N}_{\eta, n+\alpha}$ are given by, 


$$
\begin{aligned}
& \mathbf{N}_{\xi, n+\alpha}:=\left[\mathbf{N}_{\xi_{1}, n+\alpha}, \ldots, \mathbf{N}_{\xi_{\text {mnod }}, n+\alpha}\right], \quad \mathbf{N}_{\xi_{A}, n+\alpha}:=\left.\frac{\partial N_{A}(\boldsymbol{\xi})}{\partial \xi}\right|_{\bar{\xi}_{n+\alpha}} \mathbf{1} \\
& \mathbf{N}_{\eta, n+\alpha}:=\left[\mathbf{N}_{\eta_{1}, n+\alpha}, \ldots, \mathbf{N}_{\eta_{\text {mood }}, n+\alpha}\right], \quad \mathbf{N}_{\eta_{A}, n+\alpha}:=\left.\frac{\partial N_{A}(\boldsymbol{\xi})}{\partial \eta}\right|_{\bar{\xi}_{n+\alpha}} \mathbf{1}
\end{aligned}
$$

The outward unit normal vector $\mathbf{n}_{n+\alpha}$ to the master surface at the configuration $n+\alpha$ of the closest-point-projection can be defined as,

$$
\mathbf{n}_{n+\alpha}=\frac{\boldsymbol{\tau}_{\xi, n+\alpha} \times \tau_{\eta, n+\alpha}}{\left\|\tau_{\xi, n+\alpha} \times \tau_{\eta, n+\alpha}\right\|}
$$

Let us introduce the normalized covariant tangent vectors $\hat{\tau}_{\xi, n+\alpha}$ and $\hat{\tau}_{\eta, n+\alpha}$ defined as,

$$
\hat{\boldsymbol{\tau}}_{\xi, n+\alpha}:=\frac{\boldsymbol{\tau}_{\xi, n+\alpha}}{\left\|\boldsymbol{\tau}_{\xi, n+\alpha}\right\|}, \quad \hat{\boldsymbol{\tau}}_{\eta, n+\alpha}:=\frac{\boldsymbol{\tau}_{\eta, n+\alpha}}{\left\|\tau_{\eta, n+\alpha}\right\|}
$$

and orthogonal unit tangent vectors $\tau_{1, n+\alpha}$ and $\tau_{2, n+\alpha}$ defined as,

$$
\begin{aligned}
& \tau_{1, n+\alpha}:=\hat{\tau}_{\xi, n+\alpha} \\
& \tau_{2, n+\alpha}:=\mathbf{n}_{n+\alpha} \times \tau_{1, n+\alpha}=-\tau_{1, n+\alpha} \times \mathbf{n}_{n+\alpha}
\end{aligned}
$$

The variation of the covariant tangent vectors $\tau_{\xi, n+\alpha}$ and $\tau_{\eta, n+\alpha}$ given by (82) takes the form,

$$
\begin{aligned}
& \Delta \boldsymbol{\tau}_{\xi, n+\alpha}=\mathbf{N}_{\xi, n+\alpha} \Delta \mathbf{u}_{m, n+\alpha}+\Delta \mathbf{N}_{\xi, n+\alpha} \mathbf{x}_{m, n+\alpha} \\
& \Delta \tau_{\eta, n+\alpha}=\mathbf{N}_{\eta, n+\alpha} \Delta \mathbf{u}_{m, n+\alpha}+\Delta \mathbf{N}_{\eta, n+\alpha} \mathbf{x}_{m, n+\alpha}
\end{aligned}
$$

where the variations $\Delta \mathbf{N}_{\xi, n+\alpha}, \Delta \mathbf{N}_{\eta, n+\alpha}$ are given by,

$$
\begin{aligned}
\Delta \mathbf{N}_{\xi, n+\alpha} & =\mathbf{N}_{\xi \xi, n+\alpha} \Delta \bar{\xi}_{n+\alpha}+\mathbf{N}_{\xi \eta, n+\alpha} \Delta \bar{\eta}_{n+\alpha} \\
\Delta \mathbf{N}_{\eta, n+\alpha} & =\mathbf{N}_{\eta \xi, n+\alpha} \Delta \bar{\xi}_{n+\alpha}+\mathbf{N}_{\eta \eta, n+\alpha} \Delta \bar{\eta}_{n+\alpha}
\end{aligned}
$$

where the $n_{\mathrm{dim}} \times n_{\mathrm{dim}} n_{\text {mnod }}$ matrix operators $\mathbf{N}_{\xi \xi, n+\alpha}, \mathbf{N}_{\xi \eta, n+\alpha}=\mathbf{N}_{\eta \xi, n+\alpha}, \mathbf{N}_{\eta \eta, n+\alpha}$ are defined as, 


$$
\begin{aligned}
& \mathbf{N}_{\xi \xi, n+\alpha}:=\left[\mathbf{N}_{\xi \xi_{1}, n+\alpha}, \ldots, \mathbf{N}_{\xi \xi_{\text {mnod }}, n+\alpha}\right], \quad \mathbf{N}_{\xi \xi_{A}, n+\alpha}:=\left.\frac{\partial^{2} N_{A}(\boldsymbol{\xi})}{\partial \xi \partial \xi}\right|_{\bar{\xi}_{n+\alpha}} \mathbf{1} \\
& \mathbf{N}_{\xi \eta, n+\alpha}:=\left[\mathbf{N}_{\xi \eta_{1}, n+\alpha}, \ldots, \mathbf{N}_{\xi \eta_{\text {mond }}, n+\alpha}\right], \quad \mathbf{N}_{\xi \eta_{A}, n+\alpha}:=\left.\frac{\partial^{2} N_{A}(\boldsymbol{\xi})}{\partial \xi \partial \eta}\right|_{\bar{\xi}_{n+\alpha}} \mathbf{1} \\
& \mathbf{N}_{\eta \eta, n+\alpha}:=\left[\mathbf{N}_{\eta \eta_{1}, n+\alpha}, \ldots, \mathbf{N}_{\eta \eta_{\text {mnod }}, n+\alpha}\right], \quad \mathbf{N}_{\eta \eta_{A}, n+\alpha}:=\left.\frac{\partial^{2} N_{A}(\boldsymbol{\xi})}{\partial \eta \partial \eta}\right|_{\bar{\xi}_{n+\alpha}} \mathbf{1}
\end{aligned}
$$

Note that for linear elements, the second derivatives of the shape functions are zero, yielding $\Delta \mathbf{N}_{\xi, n+\alpha}=\mathbf{0}$ and $\Delta \mathbf{N}_{\eta, n+\alpha}=\mathbf{0}$.

In order to get the variations of the contravariant components $\Delta \bar{\xi}_{n+\alpha}, \Delta \bar{\eta}_{n+\alpha}$ in terms of the components $\Delta u_{s 1, n+1}, \Delta u_{s 2, n+1}$ let us consider the following relationship,

$$
\Delta \bar{\xi}_{n+1} \tau_{\xi, n+\alpha}+\Delta \bar{\eta}_{n+1} \tau_{\eta, n+\alpha}=\Delta u_{s 1, n+1} \tau_{1, n+\alpha}+\Delta u_{s 2, n+1} \tau_{2, n+\alpha}
$$

and let us introduce the following geometric relationships involving the covariant and contravariant tangent vectors,

$$
\begin{gathered}
\tau_{\beta, n+\alpha} \cdot \tau_{\gamma, n+\alpha}=m_{\beta \gamma, n+\alpha}, \quad \tau_{n+\alpha}^{\beta} \cdot \tau_{n+\alpha}^{\gamma}=m_{n+\alpha}^{\beta \gamma}, \quad \tau_{\beta, n+\alpha} \cdot \tau_{n+\alpha}^{\gamma}=\delta_{\beta, n+\alpha}^{\gamma} \\
\tau_{\beta, n+\alpha}=m_{\beta \gamma, n+\alpha} \cdot \tau_{n+\alpha}^{\gamma}, \quad \tau_{n+\alpha}^{\beta}=m_{n+\alpha}^{\beta \gamma} \cdot \tau_{\gamma, n+\alpha}
\end{gathered}
$$

Multiplying (90) by the contravariant tangent vectors $\tau_{n+\alpha}^{\xi}$ and $\tau_{n+\alpha}^{\eta}$ and taking into account (91) yields the following expressions for the contravariant components $\Delta \bar{\xi}_{n+\alpha}, \Delta \bar{\eta}_{n+\alpha}$ in terms of the components $\Delta u_{s 1, n+1}, \Delta u_{s 2, n+1}$,

$$
\begin{aligned}
& \Delta \bar{\xi}_{n+1}=\Delta u_{s 1, n+1} \tau_{1, n+\alpha} \cdot \tau_{n+\alpha}^{\xi}+\Delta u_{s 2, n+1} \tau_{2, n+\alpha} \cdot \tau_{n+\alpha}^{\xi}:=m_{1, n+\alpha}^{\xi} \Delta u_{s 1, n+1}+m_{2, n+\alpha}^{\xi} \Delta u_{s 2, n+1} \\
& \Delta \bar{\eta}_{n+1}=\Delta u_{s 1, n+1} \tau_{1, n+\alpha} \cdot \tau_{n+\alpha}^{\eta}+\Delta u_{s 2, n+1} \tau_{2, n+\alpha} \cdot \tau_{n+\alpha}^{\eta}:=m_{1, n+\alpha}^{\eta} \Delta u_{s 1, n+1}+m_{2, n+\alpha}^{\eta} \Delta u_{s 2, n+1}
\end{aligned}
$$

where the metrics $m_{1, n+\alpha}^{\xi}, m_{2, n+\alpha}^{\xi}, m_{1, n+\alpha}^{\eta}, m_{2, n+\alpha}^{\eta}$ are defined as,

$$
\begin{array}{ll}
m_{1, n+\alpha}^{\xi}:=\tau_{1, n+\alpha} \cdot \tau_{n+\alpha}^{\xi}, & m_{2, n+\alpha}^{\xi}:=\tau_{2, n+\alpha} \cdot \tau_{n+\alpha}^{\xi} \\
m_{1, n+\alpha}^{\eta}:=\tau_{1, n+\alpha} \cdot \tau_{n+\alpha}^{\eta}, & m_{2, n+\alpha}^{\eta}:=\tau_{2, n+\alpha} \cdot \tau_{n+\alpha}^{\eta}
\end{array}
$$

Taking into account that $\Delta \bar{\xi}_{n+\alpha}=\alpha \Delta \bar{\xi}_{n+1}$ and $\Delta \bar{\eta}_{n+\alpha}=\alpha \Delta \bar{\eta}_{n+1}$ yields,

$$
\begin{aligned}
& \Delta \bar{\xi}_{n+\alpha}=\alpha \Delta \bar{\xi}_{n+1}=\alpha\left(m_{1, n+\alpha}^{\xi} \Delta u_{s 1, n+1}+m_{2, n+\alpha}^{\xi} \Delta u_{s 2, n+1}\right) \\
& \Delta \bar{\eta}_{n+\alpha}=\alpha \Delta \bar{\eta}_{n+1}=\alpha\left(m_{1, n+\alpha}^{\eta} \Delta u_{s 1, n+1}+m_{2, n+\alpha}^{\eta} \Delta u_{s 2, n+1}\right)
\end{aligned}
$$

Substituting (94) into (88) and (88) into (87), and taking into account that $\Delta \mathbf{u}_{m, n+\alpha}=\alpha \Delta \mathbf{u}_{m, n+1}$, the variation of the covariant tangent vectors $\tau_{\xi, n+\alpha}$ and $\tau_{\eta, n+\alpha}$ given by (82) takes the form, 


$$
\begin{aligned}
\Delta \tau_{\xi, n+\alpha} & =\mathbf{N}_{\xi, n+\alpha} \Delta \mathbf{u}_{m, n+\alpha}+\mathbf{N}_{\xi \xi, n+\alpha} \mathbf{x}_{m, n+\alpha} \Delta \bar{\xi}_{n+\alpha}+\mathbf{N}_{\xi \eta, n+\alpha} \mathbf{x}_{m, n+\alpha} \Delta \bar{\eta}_{n+\alpha} \\
= & \alpha \mathbf{N}_{\xi, n+\alpha} \Delta \mathbf{u}_{m, n+1} \\
& +\alpha \mathbf{N}_{\xi \xi, n+\alpha} \mathbf{x}_{m, n+\alpha}\left(m_{1, n+\alpha}^{\xi} \Delta u_{s 1, n+1}+m_{2, n+\alpha}^{\xi} \Delta u_{s 2, n+1}\right) \\
& +\alpha \mathbf{N}_{\xi \eta, n+\alpha} \mathbf{x}_{m, n+\alpha}\left(m_{1, n+\alpha}^{\eta} \Delta u_{s 1, n+1}+m_{2, n+\alpha}^{\eta} \Delta u_{s 2, n+1}\right) \\
= & \alpha \mathbf{N}_{\xi, n+\alpha} \Delta \mathbf{u}_{m, n+1} \\
& +\alpha\left(\mathbf{N}_{\xi \xi, n+\alpha} m_{1, n+\alpha}^{\xi}+\mathbf{N}_{\xi \eta, n+\alpha} m_{1, n+\alpha}^{\eta}\right) \mathbf{x}_{m, n+\alpha} \Delta u_{s 1, n+1} \\
& +\alpha\left(\mathbf{N}_{\xi \xi, n+\alpha} m_{2, n+\alpha}^{\xi}+\mathbf{N}_{\xi \eta, n+\alpha} m_{2, n+\alpha}^{\eta}\right) \mathbf{x}_{m, n+\alpha} \Delta u_{s 2, n+1} \\
& =\mathbf{N}_{\eta, n+\alpha} \Delta \mathbf{u}_{m, n+\alpha}+\mathbf{N}_{\eta \xi, n+\alpha} \mathbf{x}_{m, n+\alpha} \Delta \bar{\xi}_{n+\alpha}+\mathbf{N}_{\eta \eta, n+\alpha} \mathbf{x}_{m, n+\alpha} \Delta \bar{\eta}_{n+\alpha} \\
= & \alpha \mathbf{N}_{\eta, n+\alpha} \Delta \mathbf{u}_{m, n+1} \\
& +\alpha \mathbf{N}_{\eta \xi, n+\alpha} \mathbf{x}_{m, n+\alpha}\left(m_{1, n+\alpha}^{\xi} \Delta u_{s 1, n+1}+m_{2, n+\alpha}^{\xi} \Delta u_{s 2, n+1}\right) \\
& +\alpha \mathbf{N}_{\eta \eta, n+\alpha} \mathbf{x}_{m, n+\alpha}\left(m_{1, n+\alpha}^{\eta} \Delta u_{s 1, n+1}+m_{2, n+\alpha}^{\eta} \Delta u_{s 2, n+1}\right) \\
= & \alpha \mathbf{N}_{\eta, n+\alpha} \Delta \mathbf{u}_{m, n+1} \\
& +\alpha\left(\mathbf{N}_{\eta \xi, n+\alpha} m_{1, n+\alpha}^{\xi}+\mathbf{N}_{\eta \eta, n+\alpha} m_{1, n+\alpha}^{\eta}\right) \mathbf{x}_{m, n+\alpha} \Delta u_{s 1, n+1} \\
& +\alpha\left(\mathbf{N}_{\eta \xi, n+\alpha} m_{2, n+\alpha}^{\xi}+\mathbf{N}_{\eta \eta, n+\alpha} m_{2, n+\alpha}^{\eta}\right) \mathbf{x}_{m, n+\alpha} \Delta u_{s 2, n+1}
\end{aligned}
$$

Let us introduce the vector operators $\mathbf{N}_{\xi 1, n+\alpha}, \mathbf{N}_{\xi 2, n+\alpha}, \mathbf{N}_{\eta 1, n+\alpha}, \mathbf{N}_{\eta 2, n+\alpha}$ defined as,

$$
\begin{aligned}
& \mathbf{N}_{\xi 1, n+\alpha}:=\left(\mathbf{N}_{\xi \xi, n+\alpha} m_{1, n+\alpha}^{\xi}+\mathbf{N}_{\xi \eta, n+\alpha} m_{1, n+\alpha}^{\eta}\right) \mathbf{x}_{m, n+\alpha} \\
& \mathbf{N}_{\xi 2, n+\alpha}:=\left(\mathbf{N}_{\xi \xi, n+\alpha} m_{2, n+\alpha}^{\xi}+\mathbf{N}_{\xi \eta, n+\alpha} m_{2, n+\alpha}^{\eta}\right) \mathbf{x}_{m, n+\alpha} \\
& \mathbf{N}_{\eta 1, n+\alpha}:=\left(\mathbf{N}_{\eta \xi, n+\alpha} m_{1, n+\alpha}^{\xi}+\mathbf{N}_{\eta \eta, n+\alpha} m_{1, n+\alpha}^{\eta}\right) \mathbf{x}_{m, n+\alpha} \\
& \mathbf{N}_{\eta 2, n+\alpha}:=\left(\mathbf{N}_{\eta \xi, n+\alpha} m_{2, n+\alpha}^{\xi}+\mathbf{N}_{\eta \eta, n+\alpha} m_{2, n+\alpha}^{\eta}\right) \mathbf{x}_{m, n+\alpha}
\end{aligned}
$$

Using the vector operators introduced in (96), the variation of the covariant tangent vectors $\tau_{\xi, n+\alpha}$ and $\tau_{\eta, n+\alpha}$ given by (82) takes the form,

$$
\begin{aligned}
& \Delta \boldsymbol{\tau}_{\xi, n+\alpha}:=\alpha\left(\mathbf{N}_{\xi, n+\alpha} \Delta \mathbf{u}_{m, n+1}+\mathbf{N}_{\xi 1, n+\alpha} \Delta u_{s 1, n+1}+\mathbf{N}_{\xi 2, n+\alpha} \Delta u_{s 2, n+1}\right) \\
& \Delta \tau_{\eta, n+\alpha}:=\alpha\left(\mathbf{N}_{\eta, n+\alpha} \Delta \mathbf{u}_{m, n+1}+\mathbf{N}_{\eta 1, n+\alpha} \Delta u_{s 1, n+1}+\mathbf{N}_{\eta 2, n+\alpha} \Delta u_{s 2, n+1}\right)
\end{aligned}
$$

Introducing the vector $\Delta \mathbf{u}_{s, n+1}^{\prime}$ defined as,

$$
\Delta \mathbf{u}_{s, n+1}^{\prime}:=\left[\Delta u_{s 1, n+1}, \Delta u_{s 2, n+1}, 0\right]^{T}
$$

and the matrix operators $\overline{\mathbf{N}}_{\xi, n+\alpha}, \overline{\mathbf{N}}_{\eta, n+\alpha}$ defined as, 


$$
\begin{aligned}
& \overline{\mathbf{N}}_{\xi, n+\alpha}:=\left[\mathbf{N}_{\xi 1, n+\alpha}, \mathbf{N}_{\xi 2, n+\alpha}, \mathbf{0}\right] \\
& \overline{\mathbf{N}}_{\eta, n+\alpha}:=\left[\mathbf{N}_{\eta 1, n+\alpha}, \mathbf{N}_{\eta 2, n+\alpha}, \mathbf{0}\right]
\end{aligned}
$$

the variation of the covariant tangent vectors $\tau_{\xi, n+\alpha}$ and $\tau_{\eta, n+\alpha}$ given by (82) takes the form,

$$
\begin{aligned}
& \Delta \boldsymbol{\tau}_{\xi, n+\alpha}:=\alpha\left(\mathbf{N}_{\xi, n+\alpha} \Delta \mathbf{u}_{m, n+1}+\overline{\mathbf{N}}_{\xi, n+\alpha} \Delta \mathbf{u}_{s, n+1}^{\prime}\right) \\
& \Delta \boldsymbol{\tau}_{\eta, n+\alpha}:=\alpha\left(\mathbf{N}_{\eta, n+\alpha} \Delta \mathbf{u}_{m, n+1}+\overline{\mathbf{N}}_{\eta, n+\alpha} \Delta \mathbf{u}_{s, n+1}^{\prime}\right)
\end{aligned}
$$

The variation of the normalized covariant tangent vectors $\hat{\tau}_{\xi, n+\alpha}$ and $\hat{\tau}_{\eta, n+\alpha}$ given by (85) takes the form,

$$
\begin{aligned}
\Delta \hat{\boldsymbol{\tau}}_{\xi, n+\alpha} & =\frac{1}{\left\|\boldsymbol{\tau}_{\xi, n+\alpha}\right\|}\left(\mathbf{1}-\hat{\boldsymbol{\tau}}_{\xi, n+\alpha} \otimes \hat{\boldsymbol{\tau}}_{\xi, n+\alpha}\right) \Delta \boldsymbol{\tau}_{\xi, n+\alpha}:=\frac{1}{\left\|\boldsymbol{\tau}_{\xi, n+\alpha}\right\|} \mathbf{P}_{\xi, n+\alpha} \Delta \boldsymbol{\tau}_{\xi, n+\alpha} \\
\Delta \hat{\boldsymbol{\tau}}_{\eta, n+\alpha} & =\frac{1}{\left\|\boldsymbol{\tau}_{\eta, n+\alpha}\right\|}\left(\mathbf{1}-\hat{\boldsymbol{\tau}}_{\eta, n+\alpha} \otimes \hat{\boldsymbol{\tau}}_{\eta, n+\alpha}\right) \Delta \boldsymbol{\tau}_{\eta, n+\alpha}:=\frac{1}{\left\|\boldsymbol{\tau}_{\eta, n+\alpha}\right\|} \mathbf{P}_{\eta, n+\alpha} \Delta \boldsymbol{\tau}_{\eta, n+\alpha}
\end{aligned}
$$

where we have introduced the second order projection operators $\mathbf{P}_{\xi, n+\alpha}, \mathbf{P}_{\eta, n+\alpha}$ defined as,

$$
\begin{aligned}
& \mathbf{P}_{\xi, n+\alpha}:=\mathbf{1}-\hat{\boldsymbol{\tau}}_{\xi, n+\alpha} \otimes \hat{\boldsymbol{\tau}}_{\xi, n+\alpha} \\
& \mathbf{P}_{\eta, n+\alpha}:=\mathbf{1}-\hat{\boldsymbol{\tau}}_{\eta, n+\alpha} \otimes \hat{\boldsymbol{\tau}}_{\eta, n+\alpha}
\end{aligned}
$$

Substituting (97) into (101), the variation of the normalized covariant tangent vectors $\hat{\tau}_{\xi, n+\alpha}$ and $\hat{\tau}_{\eta, n+\alpha}$ given by (85) takes the form,

$$
\begin{aligned}
& \Delta \hat{\boldsymbol{\tau}}_{\xi, n+\alpha}=\frac{1}{\left\|\tau_{\xi, n+\alpha}\right\|} \mathbf{P}_{\xi, n+\alpha} \alpha\left(\mathbf{N}_{\xi, n+\alpha} \Delta \mathbf{u}_{m, n+1}+\mathbf{N}_{\xi 1, n+\alpha} \Delta u_{s 1, n+1}+\mathbf{N}_{\xi 2, n+\alpha} \Delta u_{s 2, n+1}\right) \\
& \Delta \hat{\tau}_{\eta, n+\alpha}=\frac{1}{\left\|\tau_{\eta, n+\alpha}\right\|} \mathbf{P}_{\eta, n+\alpha} \alpha\left(\mathbf{N}_{\eta, n+\alpha} \Delta \mathbf{u}_{m, n+1}+\mathbf{N}_{\eta 1, n+\alpha} \Delta u_{s 1, n+1}+\mathbf{N}_{\eta 2, n+\alpha} \Delta u_{s 2, n+1}\right)
\end{aligned}
$$

Similarly, substituting (100) into (101), the variation of the normalized covariant tangent vectors $\hat{\tau}_{\xi, n+\alpha}$ and $\hat{\tau}_{\eta, n+\alpha}$ given by (85) takes the form,

$$
\begin{aligned}
& \Delta \hat{\boldsymbol{\tau}}_{\xi, n+\alpha}=\frac{1}{\left\|\boldsymbol{\tau}_{\xi, n+\alpha}\right\|} \mathbf{P}_{\xi, n+\alpha} \alpha\left(\mathbf{N}_{\xi, n+\alpha} \Delta \mathbf{u}_{m, n+1}+\overline{\mathbf{N}}_{\xi, n+\alpha} \Delta \mathbf{u}_{s, n+1}^{\prime}\right) \\
& \Delta \hat{\tau}_{\eta, n+\alpha}=\frac{1}{\left\|\boldsymbol{\tau}_{\eta, n+\alpha}\right\|} \mathbf{P}_{\eta, n+\alpha} \alpha\left(\mathbf{N}_{\eta, n+\alpha} \Delta \mathbf{u}_{m, n+1}+\overline{\mathbf{N}}_{\eta, n+\alpha} \Delta \mathbf{u}_{s, n+1}^{\prime}\right)
\end{aligned}
$$

Introducing the matrix operators $\mathbf{A}_{\xi, n+\alpha}, \mathbf{A}_{\eta, n+\alpha}, \overline{\mathbf{A}}_{\xi, n+\alpha}, \overline{\mathbf{A}}_{\eta, n+\alpha}$ defined as, 


$$
\begin{array}{ll}
\mathbf{A}_{\xi, n+\alpha}:=\frac{1}{\left\|\boldsymbol{\tau}_{\xi, n+\alpha}\right\|} \mathbf{P}_{\xi, n+\alpha} \mathbf{N}_{\xi, n+\alpha}, \quad \mathbf{A}_{\eta, n+\alpha}:=\frac{1}{\left\|\tau_{\eta, n+\alpha}\right\|} \mathbf{P}_{\eta, n+\alpha} \mathbf{N}_{\eta, n+\alpha} \\
\overline{\mathbf{A}}_{\xi, n+\alpha}:=\frac{1}{\left\|\boldsymbol{\tau}_{\xi, n+\alpha}\right\|} \mathbf{P}_{\xi, n+\alpha} \overline{\mathbf{N}}_{\xi, n+\alpha}, \quad \overline{\mathbf{A}}_{\eta, n+\alpha}:=\frac{1}{\left\|\boldsymbol{\tau}_{\xi, n+\alpha}\right\|} \mathbf{P}_{\eta, n+\alpha} \overline{\mathbf{N}}_{\eta, n+\alpha}
\end{array}
$$

the variation of the normalized covariant tangent vectors $\hat{\tau}_{\xi, n+\alpha}$ and $\hat{\tau}_{\eta, n+\alpha}$ given by (85) takes the form,

$$
\begin{aligned}
& \Delta \hat{\boldsymbol{\tau}}_{\xi, n+\alpha}:=\alpha\left(\mathbf{A}_{\xi, n+\alpha} \Delta \mathbf{u}_{m, n+1}+\overline{\mathbf{A}}_{\xi, n+\alpha} \Delta \mathbf{u}_{s, n+1}^{\prime}\right) \\
& \Delta \hat{\boldsymbol{\tau}}_{\eta, n+\alpha}:=\alpha\left(\mathbf{A}_{\eta, n+\alpha} \Delta \mathbf{u}_{m, n+1}+\overline{\mathbf{A}}_{\eta, n+\alpha} \Delta \mathbf{u}_{s, n+1}^{\prime}\right)
\end{aligned}
$$

The variations of the outward unit normal vector $\mathbf{n}_{n+\alpha}$ given by (84) and the orthogonal unit tangent vectors $\tau_{1, n+\alpha}$ and $\tau_{2, n+\alpha}$ given by (86) take the form,

$$
\begin{aligned}
\Delta \mathbf{n}_{n+\alpha} & =\frac{1}{\left\|\tau_{\xi, n+\alpha} \times \tau_{\eta, n+\alpha}\right\|} \mathbf{P}_{n+\alpha}\left(\boldsymbol{\tau}_{\xi, n+\alpha} \times \Delta \tau_{\eta, n+\alpha}+\Delta \tau_{\xi, n+\alpha} \times \tau_{\eta, n+\alpha}\right) \\
\Delta \tau_{1, n+\alpha} & =\Delta \hat{\boldsymbol{\tau}}_{\xi, n+\alpha} \\
\Delta \boldsymbol{\tau}_{2, n+\alpha} & =\mathbf{n}_{n+\alpha} \times \Delta \hat{\tau}_{\xi, n+\alpha}+\Delta \mathbf{n}_{n+\alpha} \times \hat{\boldsymbol{\tau}}_{\xi, n+\alpha} \\
& =\mathbf{n}_{n+\alpha} \times \Delta \hat{\boldsymbol{\tau}}_{\xi, n+\alpha} \\
& +\frac{1}{\left\|\boldsymbol{\tau}_{\xi, n+\alpha} \times \boldsymbol{\tau}_{\eta, n+\alpha}\right\|} \mathbf{P}_{n+\alpha}\left(\boldsymbol{\tau}_{\xi, n+\alpha} \times \Delta \boldsymbol{\tau}_{\eta, n+\alpha}\right) \times \hat{\boldsymbol{\tau}}_{\xi, n+\alpha} \\
& +\frac{1}{\left\|\boldsymbol{\tau}_{\xi, n+\alpha} \times \boldsymbol{\tau}_{\eta, n+\alpha}\right\|} \mathbf{P}_{n+\alpha}\left(\Delta \boldsymbol{\tau}_{\xi, n+\alpha} \times \boldsymbol{\tau}_{\eta, n+\alpha}\right) \times \hat{\boldsymbol{\tau}}_{\xi, n+\alpha}
\end{aligned}
$$

where the second order projection operator $\mathbf{P}_{n+\alpha}$ has been defined as,

$$
\mathbf{P}_{n+\alpha}:=\mathbf{1}-\mathbf{n}_{n+\alpha} \otimes \mathbf{n}_{n+\alpha}
$$

Let us introduce a skew-symmetric second order tensor $\mathbf{w}(\mathbf{a})$ such that the following expressions hold for any vector $\mathbf{a}, \mathbf{b}, \mathbf{c}$,

$$
\begin{aligned}
\mathbf{w}(\mathbf{a}) \mathbf{b} & =\mathbf{a} \times \mathbf{b}=-\mathbf{b} \times \mathbf{a} \\
\mathbf{w}(\mathbf{a}) \mathbf{w}(\mathbf{b}) \mathbf{c}=\mathbf{a} \times(\mathbf{b} \times \mathbf{c}) & =-(\mathbf{b} \times \mathbf{c}) \times \mathbf{a}=(\mathbf{a} \cdot \mathbf{c}) \mathbf{b}-(\mathbf{a} \cdot \mathbf{b}) \mathbf{c}
\end{aligned}
$$

where the components of the skew-symmetric matrix $\mathbf{w}(\mathbf{a})$ are written in terms of the components of the vector a as,

$$
[\mathbf{w}(\mathbf{a})]:=\left[\begin{array}{ccc}
0 & -a_{3} & a_{2} \\
a_{3} & 0 & -a_{1} \\
-a_{2} & a_{1} & 0
\end{array}\right], \quad[\mathbf{a}]:=\left[\begin{array}{l}
a_{1} \\
a_{2} \\
a_{3}
\end{array}\right]
$$


Then, the variations of the outward unit normal vector $\mathbf{n}_{n+\alpha}$ given by (84) and orthogonal unit tangent vectors $\tau_{1, n+\alpha}$ and $\tau_{2, n+\alpha}$ given by (86) take the form,

$$
\begin{aligned}
\Delta \mathbf{n}_{n+\alpha}= & \frac{1}{\left\|\boldsymbol{\tau}_{\xi, n+\alpha} \times \boldsymbol{\tau}_{\eta, n+\alpha}\right\|} \mathbf{P}_{n+\alpha}\left(\mathbf{w}\left(\boldsymbol{\tau}_{\xi, n+\alpha}\right) \Delta \boldsymbol{\tau}_{\eta, n+\alpha}-\mathbf{w}\left(\boldsymbol{\tau}_{\eta, n+\alpha}\right) \Delta \boldsymbol{\tau}_{\xi, n+\alpha}\right) \\
\Delta \boldsymbol{\tau}_{1, n+\alpha}= & \Delta \hat{\boldsymbol{\tau}}_{\xi, n+\alpha} \\
\Delta \boldsymbol{\tau}_{2, n+\alpha}= & \mathbf{w}\left(\mathbf{n}_{n+\alpha}\right) \Delta \hat{\tau}_{\xi, n+\alpha}-\mathbf{w}\left(\hat{\tau}_{\xi, n+\alpha}\right) \Delta \mathbf{n}_{n+\alpha} \\
= & \mathbf{w}\left(\mathbf{n}_{n+\alpha}\right) \Delta \hat{\boldsymbol{\tau}}_{\xi, n+\alpha} \\
& -\frac{1}{\left\|\boldsymbol{\tau}_{\xi, n+\alpha} \times \boldsymbol{\tau}_{\eta, n+\alpha}\right\|} \mathbf{w}\left(\hat{\boldsymbol{\tau}}_{\xi, n+\alpha}\right) \mathbf{P}_{n+\alpha} \mathbf{w}\left(\boldsymbol{\tau}_{\xi, n+\alpha}\right) \Delta \boldsymbol{\tau}_{\eta, n+\alpha} \\
& +\frac{1}{\left\|\tau_{\xi, n+\alpha} \times \boldsymbol{\tau}_{\eta, n+\alpha}\right\|} \mathbf{w}\left(\hat{\tau}_{\xi, n+\alpha}\right) \mathbf{P}_{n+\alpha} \mathbf{w}\left(\boldsymbol{\tau}_{\eta, n+\alpha}\right) \Delta \boldsymbol{\tau}_{\xi, n+\alpha}
\end{aligned}
$$

Using (100) and (106) the variations of the outward unit normal vector $\mathbf{n}_{n+\alpha}$ given by (84) and orthogonal unit tangent vectors $\tau_{1, n+\alpha}$ and $\tau_{2, n+\alpha}$ given by (86) take the form,

$$
\begin{aligned}
\Delta \mathbf{n}_{n+\alpha}= & \frac{1}{\left\|\boldsymbol{\tau}_{\xi, n+\alpha} \times \tau_{\eta, n+\alpha}\right\|} \mathbf{P}_{n+\alpha}\left(\mathbf{w}\left(\boldsymbol{\tau}_{\xi, n+\alpha}\right) \mathbf{N}_{\eta, n+\alpha}-\mathbf{w}\left(\boldsymbol{\tau}_{\eta, n+\alpha}\right) \mathbf{N}_{\xi, n+\alpha}\right) \Delta \mathbf{u}_{m, n+\alpha} \\
& +\frac{1}{\left\|\tau_{\xi, n+\alpha} \times \tau_{\eta, n+\alpha}\right\|} \mathbf{P}_{n+\alpha}\left(\mathbf{w}\left(\boldsymbol{\tau}_{\xi, n+\alpha}\right) \overline{\mathbf{N}}_{\eta, n+\alpha}-\mathbf{w}\left(\boldsymbol{\tau}_{\eta, n+\alpha}\right) \overline{\mathbf{N}}_{\xi, n+\alpha}\right) \Delta \mathbf{u}_{s, n+\alpha}^{\prime} \\
\Delta \tau_{1, n+\alpha}= & \mathbf{A}_{\xi, n+\alpha} \Delta \mathbf{u}_{m, n+\alpha}+\overline{\mathbf{A}}_{\xi, n+\alpha} \Delta \mathbf{u}_{s, n+\alpha}^{\prime} \\
\Delta \boldsymbol{\tau}_{2, n+\alpha}= & \mathbf{w}\left(\mathbf{n}_{n+\alpha}\right)\left(\mathbf{A}_{\xi, n+\alpha} \Delta \mathbf{u}_{m, n+\alpha}+\overline{\mathbf{A}}_{\xi, n+\alpha} \Delta \mathbf{u}_{s, n+\alpha}^{\prime}\right) \\
& -\frac{1}{\left\|\tau_{\xi, n+\alpha} \times \tau_{\eta, n+\alpha}\right\|} \mathbf{w}\left(\hat{\tau}_{\xi, n+\alpha}\right) \mathbf{P}_{n+\alpha} \mathbf{w}\left(\boldsymbol{\tau}_{\xi, n+\alpha}\right)\left(\mathbf{N}_{\eta, n+\alpha} \Delta \mathbf{u}_{m, n+\alpha}+\overline{\mathbf{N}}_{\eta, n+\alpha} \Delta \mathbf{u}_{s, n+\alpha}^{\prime}\right) \\
& +\frac{1}{\left\|\boldsymbol{\tau}_{\xi, n+\alpha} \times \tau_{\eta, n+\alpha}\right\|} \mathbf{w}\left(\hat{\tau}_{\xi, n+\alpha}\right) \mathbf{P}_{n+\alpha} \mathbf{w}\left(\tau_{\eta, n+\alpha}\right)\left(\mathbf{N}_{\xi, n+\alpha} \Delta \mathbf{u}_{m, n+\alpha}+\overline{\mathbf{N}}_{\xi, n+\alpha} \Delta \mathbf{u}_{s, n+\alpha}^{\prime}\right) \\
= & \left(\mathbf{w}\left(\mathbf{n}_{n+\alpha}\right) \mathbf{A}_{\xi, n+\alpha}-\mathbf{w}\left(\hat{\tau}_{\xi, n+\alpha}\right) \mathbf{A}_{3, n+\alpha}\right) \Delta \mathbf{u}_{m, n+\alpha} \\
& +\left(\mathbf{w}\left(\mathbf{n}_{n+\alpha}\right) \overline{\mathbf{A}}_{\xi, n+\alpha}-\mathbf{w}\left(\hat{\boldsymbol{\tau}}_{\xi, n+\alpha}\right) \overline{\mathbf{A}}_{3, n+\alpha}\right) \Delta \mathbf{u}_{s, n+\alpha}^{\prime}
\end{aligned}
$$

Introducing the matrix operators $\mathbf{A}_{1, n+\alpha}, \mathbf{A}_{2, n+\alpha}, \mathbf{A}_{3, n+\alpha}, \overline{\mathbf{A}}_{1, n+\alpha}, \overline{\mathbf{A}}_{2, n+\alpha}, \overline{\mathbf{A}}_{3, n+\alpha}$ defined as, 


$$
\begin{aligned}
\mathbf{A}_{1, n+\alpha} & :=\mathbf{A}_{\xi, n+\alpha} \\
\mathbf{A}_{2, n+\alpha} & :=\mathbf{w}\left(\mathbf{n}_{n+\alpha}\right) \mathbf{A}_{\xi, n+\alpha}-\mathbf{w}\left(\hat{\boldsymbol{\tau}}_{\xi, n+\alpha}\right) \mathbf{A}_{3, n+\alpha} \\
\mathbf{A}_{3, n+\alpha} & :=\frac{1}{\left\|\boldsymbol{\tau}_{\xi, n+\alpha} \times \boldsymbol{\tau}_{\eta, n+\alpha}\right\|} \mathbf{P}_{n+\alpha}\left(\mathbf{w}\left(\boldsymbol{\tau}_{\xi, n+\alpha}\right) \mathbf{N}_{\eta, n+\alpha}-\mathbf{w}\left(\boldsymbol{\tau}_{\eta, n+\alpha}\right) \mathbf{N}_{\xi, n+\alpha}\right) \\
\overline{\mathbf{A}}_{1, n+\alpha} & :=\overline{\mathbf{A}}_{\xi, n+\alpha} \\
\overline{\mathbf{A}}_{2, n+\alpha} & :=\mathbf{w}\left(\mathbf{n}_{n+\alpha}\right) \overline{\mathbf{A}}_{\xi, n+\alpha}-\mathbf{w}\left(\hat{\boldsymbol{\tau}}_{\xi, n+\alpha}\right) \overline{\mathbf{A}}_{3, n+\alpha} \\
\overline{\mathbf{A}}_{3, n+\alpha} & :=\frac{1}{\left\|\boldsymbol{\tau}_{\xi, n+\alpha} \times \boldsymbol{\tau}_{\eta, n+\alpha}\right\|} \mathbf{P}_{n+\alpha}\left(\mathbf{w}\left(\boldsymbol{\tau}_{\xi, n+\alpha}\right) \overline{\mathbf{N}}_{\eta, n+\alpha}-\mathbf{w}\left(\boldsymbol{\tau}_{\eta, n+\alpha}\right) \overline{\mathbf{N}}_{\xi, n+\alpha}\right)
\end{aligned}
$$

the variations of the outward unit normal vector $\mathbf{n}_{n+\alpha}$ given by (84) and orthogonal unit tangent vectors $\tau_{1, n+\alpha}$ and $\tau_{2, n+\alpha}$ given by (86) take the form,

$$
\begin{aligned}
& \Delta \mathbf{n}_{n+\alpha}:=\mathbf{A}_{3, n+\alpha} \Delta \mathbf{u}_{m, n+\alpha}+\overline{\mathbf{A}}_{3, n+\alpha} \Delta \mathbf{u}_{s, n+\alpha}^{\prime}=\alpha\left(\mathbf{A}_{3, n+\alpha} \Delta \mathbf{u}_{m, n+1}+\overline{\mathbf{A}}_{3, n+\alpha} \Delta \mathbf{u}_{s, n+1}^{\prime}\right) \\
& \Delta \tau_{1, n+\alpha}:=\mathbf{A}_{1, n+\alpha} \Delta \mathbf{u}_{m, n+\alpha}+\overline{\mathbf{A}}_{1, n+\alpha} \Delta \mathbf{u}_{s, n+\alpha}^{\prime}=\alpha\left(\mathbf{A}_{1, n+\alpha} \Delta \mathbf{u}_{m, n+1}+\overline{\mathbf{A}}_{1, n+\alpha} \Delta \mathbf{u}_{s, n+1}^{\prime}\right) \\
& \Delta \tau_{2, n+\alpha}:=\mathbf{A}_{2, n+\alpha} \Delta \mathbf{u}_{m, n+\alpha}+\overline{\mathbf{A}}_{2, n+\alpha} \Delta \mathbf{u}_{s, n+\alpha}^{\prime}=\alpha\left(\mathbf{A}_{2, n+\alpha} \Delta \mathbf{u}_{m, n+1}+\overline{\mathbf{A}}_{2, n+\alpha} \Delta \mathbf{u}_{s, n+1}^{\prime}\right)
\end{aligned}
$$

\section{References}

1. C. Agelet de Saracibar (1997), A new frictional time integration algorithm for large slip multi-body frictional contact problems, Computer Methods in Applied Mechanics and Engineering 142, 303-334.

2. C. Agelet de Saracibar (1998), Numerical analysis of coupled thermomechanical contact problems. Computational model and applications, Archives of Computational Methods in Mechanics 5, 243-301. doi: 10.1007/BF02897875.

3. C. Agelet de Saracibar, M. Cervera, M. Chiumenti (1999), On the formulation of coupled thermoplastic problems with phase-change, International Journal of Plasticity 15, 1-34. doi: 10.1016/S0749-6419(98)00055-2.

4. C. Agelet de Saracibar, M. Chiumenti (1999), On the numerical modeling of frictional wear phenomena, Computer Methods in Applied Mechanics and Engineering 177, 401-426. doi: 10.1016/S0045-7825(98)00390-9.

5. C. Agelet de Saracibar, M. Cervera, M. Chiumenti (2001), On the constitutive modeling of coupled thermomechanical phase-change problems, International Journal of Plasticity 17, 1565-1622. doi: 10.1016/S0749-6419(00)00094-2.

6. F. Armero, E. Petocz (1998), Formulation and analysis of conserving algorithms for frictionless dynamic contact/impact problems, Computer Methods in Applied Mechanics and Engineering 158, 269-300.

7. F. Armero, E. Petocz (1999), A new dissipative time-stepping algorithm for frictional contact problems: Formulation and analysis, Computer Methods in Applied Mechanics and Engineering 179, 151-178.

8. F. Armero, I. Romero (2001), On the formulation of high-frequency dissipative timestepping algorithms for nonlinear dynamics. Part I: low-order methods for two model problems and nonlinear elastodynamics, Computer Methods in Applied Mechanics and Engineering 190, 2603-2649. 
9. F. Armero (2008), Energy-Momentum Algorithms for the Nonlinear Dynamics of Elastoplastic Solids, IUTAM Symposium on Theoretical, Computational and Modelling Aspects of Inelastic Media, IUTAM Book Series, Volume 11, 2008, pp. 251-262.

10. T. Belytschko, M.O. Neal (1991), Contact-impact by the pinball algorithm with penalty and Lagrangian methods, International Journal for Numerical Methods in Engineering 31, 547 572.

11. D.J. Benson, J.O. Hallquist (1990), A single surface contact algorithm for the post-buckling analysis of shell structures, Computer Methods in Applied Mechanics and Engineering 78, 141-163.

12. P. Betsch (2005), The discrete null space method for the energy consistent integration of constrained mechanical systems. Part I: Holonomic constraints, Computer Methods in Applied Mechanics and Engineering 194, 5159-5190.

13. R. Bravo, J.L. Pérez Aparicio, T.A. Laursen (2011), An enhanced energy conserving time stepping algorithm for frictionless particle contacts, International Journal for Numerical Methods in Engineering 85, 1415-1435.

14. S. Brunssen, S. Hartmann, E. Ramm, B. Wohlmuth (2007), Unilateral non-linear dynamic contact of thin-walled structures using a primal-dual active set strategy, International Journal for Numerical Methods Engineering 70, 883-912.

15. V. Chawla (1997), Energy Consistent Algorithms in Computational Contact Mechanics, Ph.D. Dissertation, Duke University.

16. V. Chawla, T.A. Laursen (1998), Energy consistent algorithms for frictional contact problems, International Journal for Numerical Methods in Engineering 42, 799-827.

17. M. Chiumenti, C. Agelet de Saracibar, M. Cervera (2008), On the numerical modeling of the thermomechanical contact for metal casting analysis, Journal of Heat Transfer 130, Art. No. 061301.1-10. doi: 10.1115/1.2897923.

18. A. Curnier, P. Alart (1988), A generalized Newton method for contact problems with friction, Journal de Mécanique Théorique et Appliquée, Suppl. 1, Vol. 7, 67-82.

19. G. Duvaut, J.L. Lions (1972), Les Inequations en Mecanique et en Physique, Dunod, Paris, 1972.

20. A.E. Giannakopoulos (1989), The return mapping method for the integration of friction constitutive relations, Computers and Structures, 32, 157-167.

21. O. Gonzalez (2000), Exact energy-momentum conserving algorithms for general models in nonlinear elasticity, Computer Methods in Applied Mechanics and Engineering 190, 17631783.

22. J.O. Hallquist, G.L. Goudreau, D. J. Benson (1985), Sliding interfaces with contact-impact in large-scale Lagrangian computations, Computer Methods in Applied Mechanics and Engineering 51, 107-13.

23. H. Hertz (1881), Über die Berührung fester elastischer Körper, Journal für die reine und angewandte Mathematik 92, 156-171.

24. S. Hüeber, B. Wohlmuth (2005), A primal-dual active set strategy for non-linear multibody contact problems, Computer Methods in Applied Mechanics and Engineering 194, 3147 3155 .

25. T.J.R. Hughes, J.A. Cottrell, Y. Bazilevs (2005), Isogeometric analysis: CAD, finite elements, NURBS, exact geometry and mesh refinement, Computer Methods in Applied Mechanics and Engineering 194, 4135-4195.

26. J.W. Ju, R.L. Taylor (1988), A perturbed Lagrangian formulation for the finite element solution of nonlinear frictional contact problems, Journal de Mécanique Théorique et Appliquée, Suppl. 1, Vol. 7, 1-14.

27. N. Kikuchi, J.T. Oden (1988), Contact Problems in Elasticity: A Study of Variational Inequalities and Finite Element Methods, SIAM, Philadelphia, 1988. 
28. A.W.A. Konter (2005), Advanced Finite Element Contact Benchmarks, Report: FENETUNOTT-DLE-09, Project: FENET EU Thematic Network.

29. T.A. Laursen, J.C. Simo (1991), On the formulation and numerical treatment of finite deformation frictional contact problems, in P. Wriggers and W. Wagner (eds), Nonlinear Computational Mechanics - State of the Art, Springer-Verlag, Berlin, 1991, pp. 716-736.

30. T.A. Laursen (1992), Formulation and Treatment of Frictional Contact Problems Using Finite Elements, Ph.D. Dissertation, Department of Mechanical Engineering, Stanford University.

31. T.A. Laursen, J.C. Simo (1993), A continuum-based finite element formulation for the implicit solution of multibody, large deformation frictional contact problems, International Journal for Numerical Methods in Engineering 36, 3451-3485.

32. T.A. Laursen, J.C. Simo (1993), Algorithmic symmetrization of Coulomb frictional problems using augmented Lagrangians, Computer Methods in Applied Mechanics and Engineering 108, 133-146.

33. T.A. Laursen, S. Govindjee (1994), A note on the treatment of frictionless contact between non-smooth surfaces in fully non-linear problems, Communications in Applied Numerical Methods, 10, 869-878.

34. T.A. Laursen, V. Chawla (1997), Design of energy conserving algorithms for frictionless dynamic contact problems, International Journal for Numerical Methods in Engineering 40, 863-886.

35. T.A. Laursen (1999), On the development of thermodynamically consistent algorithms for thermomechanical frictional contact, Computer Methods in Applied Mechanics and Engineering 48, 1525-1547.

36. T.A. Laursen, X.N. Meng (2001), A new solution procedure for application of energyconserving algorithms to general constitutive models in nonlinear elastodynamics, Computer Methods in Applied Mechanics and Engineering 190, 6309-6322.

37. T.A. Laursen (2002), Computational Contact and Impact Mechanics, Springer.

38. L. de Lorenzis, P. Wriggers, T.J.R. Hughes (2014), Isogeometric contact: a review, ICES Report 14-09, The Institute for Computational Engineering and Sciences, The University of Texas at Austin, May 2014.

39. X.N. Meng, T.A. Laursen (2002), Energy consistent algorithms for dynamic finite deformation plasticity, Computer Methods in Applied Mechanics and Engineering 191, 1639-1675.

40. J.J. Muñoz (2008), Modelling unilateral frictionless contact using the null-space method and cubic B-spline interpolation, Computer Methods in Applied Mechanics and Engineering 197, 979-993.

41. J.T. Oden, E.B. Pires (1984), Algorithms and numerical results for finite element approximations of contact problems with non-classical friction laws, Computers and Structures, 19, 137-147.

42. J.T. Oden, J.A.C. Martins (1985), Models and computational methods for dynamic friction phenomena, Computer Methods in Applied Mechanics and Engineering, 52, 527-634.

43. H. Parisch, A consistent tangent stiffness matrix for three-dimensional nonlinear contact analysis, International Journal for Numerical Methods in Engineering 28, 1803-1812.

44. E.G. Petocz (1988), Formulation and analysis of stable time-stepping algorithms for contact problems. Ph.D. Thesis, Department of Mechanical Engineering, Stanford, University, Stanford, California, 1998.

45. M.A. Puso, T.A. Laursen (2004), A mortar segment-to-segment contact method for large deformation solid mechanics, Computer Methods in Applied Mechanics and Engineering 193, 601-629. 
46. M.A. Puso, T.A. Laursen (2004), A mortar segment-to-segment frictional contact method for large deformations, Computer Methods in Applied Mechanics and Engineering 193, 4891-4913.

47. P. Papadopoulos, R.L. Taylor (1990), A mixed formulation for the finite element solution of contact problems, UCB/SEMM Report 90/18, University of California, Berkeley.

48. RamSeries code, http://www.compassis.com/compass/en/Productos/RamSeries.

49. J.C. Simo, K.K. Wong (1991), Unconditionally stable algorithms for rigid body dynamics that exactly preserve energy and angular momentum, International Journal for Numerical Methods in Engineering 31, 19-52.

50. J.C. Simo, T.A. Laursen (1992), An augmented Lagrangian treatment of contact problems involving friction, Computers and Structures 42, 97-116.

51. J.C. Simo, N. Tarnow (1992), The discrete energy-momentum method. Conserving algorithm for non-linear elastodynamics, ZAMP 43, 757-793.

52. J.C. Simo, N. Tarnow, K.K. Wong (1992), Exact energy-momentum conserving algorithms and symplectic schemes for nonlinear dynamics, Computer Methods in Applied Mechanics and Engineering 1, 63-116.

53. J.C. Simo (1994), Numerical Analysis Aspects of Plasticity, in P. Ciarlet and J.L. Lions (eds), Handbook of Numerical Analysis, Vol. IV, North-Holland, 1994.

54. J.C. Simo, N. Tarnow (1994), A new energy and momentum conserving algorithm for the non-linear dynamics of shells, International Journal for Numerical methods in Engineering $37,2525-2550$.

55. J.C. Simo, N. Tarnow, M. Doblaré (1995), Non-linear dynamics of three-dimensional rods: exact energy and momentum conserving algorithms, International Journal for Numerical Methods in Engineering 38, 1431-1474.

56. J.C. Simo, P. Wriggers, R.L. Taylor (1995), A perturbed Lagrangian formulation for the finite element solution of contact problems, Computer Methods in Applied Mechanics and Engineering 50, 163-180.

57. J.C. Simo, T.J.R. Hughes (1998), Computational Inelasticity, Interdisciplinary Applied Mathematics, Vol. 7, Springer, 1998.

58. C.A. Truesdell, W.M. Noll (1965), The Nonlinear Field Theories of Mechanics, Handbuch der Physik, vol. III/3, Springer, Berlin.

59. B. Wohlmuth (2000), A mortar finite element method using dual spaces for the Lagrange multiplier, SIAM Journal Numerical Analysis 38, 989-1012.

60. P. Wriggers, J.C. Simo (1985), A note on the tangent stiffness for fully nonlinear contact problems, Communications in Applied Numerical Methods, 1, 199-203.

61. P. Wriggers (1987), On consistent tangent matrices for frictional contact problems, Proceedings of the International Conference NUMETA'87, J. Middleton, G.N. Pande (eds), Nijhoff, Doredrecht, 1987.

62. P. Wriggers, T. Vu Van, E. Stein (1990), Finite element formulations of large deformation impact-contact problems with friction, Computers and Structures, 37, 319-331.

63. P. Wriggers, G. Zavarise (1993), On the application of augmented Lagrangian techniques for nonlinear constitutive laws in contact interfaces, Communications in Applied Numerical Methods, 9, 815-824.

64. P. Wriggers (1995), Finite element algorithms for contact analysis, Archives of Computational Methods in Engineering, 2, 4, 1-50.

65. B. Yang (2006), Mortar Finite Element Methods for Large Deformation Contact Mechanics, Ph.D. Dissertation, Duke University.

66. G. Zavarise, P. Wriggers, B.A. Schrefler (1995), On augmented Lagrangian algorithms for thermomechanical contact problems with friction, International Journal for Numerical Methods in Engineering 38, 2929-2949. 